\title{
Breaking the Chain: Evaluating the Links Between Opioid Use Disorder, Overdose, and Suicide
}

\author{
Amanda Nicole Stover MPH \\ West Virginia University, ans0036@mix.wvu.edu
}

Follow this and additional works at: https://researchrepository.wvu.edu/etd

Part of the Health Services Research Commons, Psychiatric and Mental Health Commons, and the Substance Abuse and Addiction Commons

\section{Recommended Citation}

Stover, Amanda Nicole MPH, "Breaking the Chain: Evaluating the Links Between Opioid Use Disorder, Overdose, and Suicide" (2020). Graduate Theses, Dissertations, and Problem Reports. 7561.

https://researchrepository.wvu.edu/etd/7561

This Dissertation is protected by copyright and/or related rights. It has been brought to you by the The Research Repository @ WVU with permission from the rights-holder(s). You are free to use this Dissertation in any way that is permitted by the copyright and related rights legislation that applies to your use. For other uses you must obtain permission from the rights-holder(s) directly, unless additional rights are indicated by a Creative Commons license in the record and/ or on the work itself. This Dissertation has been accepted for inclusion in WVU Graduate Theses, Dissertations, and Problem Reports collection by an authorized administrator of The Research Repository @ WVU.

For more information, please contact researchrepository@mail.wvu.edu. 


\title{
Breaking the Chain:
}

\section{Evaluating the Links Between Opioid Use Disorder, Overdose, and Suicide}

\author{
Amanda Nicole Stover \\ Dissertation submitted to the School of Pharmacy \\ at West Virginia University \\ in partial fulfillment of the requirements \\ for the degree of \\ Doctor in Philosophy \\ of \\ Health Services and Outcomes Research \\ Department of Pharmaceutical Systems and Policy \\ Erin L. Winstanley, PhD, Chair \\ Kimberley M. Kelly, PhD, MS \\ Traci LeMasters, $\mathbf{P h D}$ \\ Virginia G. Scott, PhD, MS,RPh \\ Ian R. H. Rockett, PhD, MPH \\ Gordon S. Smith, MD, MPH \\ Morgantown, West Virginia \\ 2020
}

Copyright 2020 Amanda Nicole Stover

Keywords: opioid use disorder, overdose, suicide, safety screening, addiction treatment, trauma 


\section{Abstract \\ Breaking the Chain: \\ Evaluating the Links Between Opioid Use Disorder, Overdose, and Suicide Amanda Nicole Stover}

Previous research has estimated that $20-30 \%$ of individuals with opioid use disorder (OUD) have a history of both suicide attempt and unintentional opioid overdose. In 2018, more than 1.9 million adults 18 years and older had an OUD; while 1.4 million adults attempted suicide that same year (SAMHSA, 2019). The key to understanding this relationship is examining behaviors that precede these potentially fatal behaviors. One such precursory behavior is self-injury. Self-injurious behaviors (SIB) are one of the most important risk factors for future suicide (Cavanagh et al.,2003). Among individuals with OUD, previous research has found that those with OUD and SIB have a standardized mortality ratio approximately 14 times greater than those without OUD (Darke \& Ross, 2002; Harris \& Barraclough, 1997; Tremeau et al., 2008; Wilcox et al., 2004).

This study has three primary objectives. The first is to conduct a systematic literature review to better understand the relationship between SIB, overdose and/or suicidal behaviors (i.e. suicidal ideation, suicide planning, or attempt) among people with OUD. The literature search was conducted in accordance with PRISMA guidelines for systematic reviews and metaanalyses. Forty-seven studies were included that examined the relationship between overdose, SIB, and/or suicidal behavior among people with OUD. Of these studies 46 were assessed using the NHLBI quality tool, $17.4 \%$ of studies were rated as poor, $32.6 \%$ were rated as fair, and $50.0 \%$ of studies were rated as good. Seven common themes were identified including: patterns 
of substance use, psychological factors/diagnoses, sociodemographic characteristics, family history, traumatic life events, gender, and methods of suicide attempt. In general, patients with OUD who are at highest risk of suicide attempt and SIB tend to have more severe psychiatric profiles, increased rates of poly-drug use, tend to be female, experience significant life stressors and childhood maltreatment.

Results from the systematic review were used to inform the second and third aims of this study. The second aim was to evaluate the feasibility of a standardized screen for suicide and overdose among patients receiving addiction treatment. This was executed by conducting a cross-sectional study among a convenience sample of patients $(\mathrm{n}=113)$ recruited from two inpatient treatment programs. A modified version of the Patient Safety Screener (mPSS) was used to screen for suicidal ideation, suicide attempt, and overdose. The screen was administered in-person during treatment and administrative clinical data were extracted. Subjects $(n=108)$ and members of their clinical care team $(n=20)$ completed a screening acceptability survey. Approximately $60 \%$ of subjects had a positive mPSS screen, and 30.3\% reported having suicidal ideation, suicide attempt, and overdose. Both subjects and clinical staff reported that it was acceptable to screen for suicide attempt(s) and overdose(s); however, a minority of clinical staff reported concerns about administration time $(n=7)$ and impact on workflow $(n=6)$.

The combined results of the systematic review and mPSS helped shape the final aim of this study. A cross-sectional study conducted in a subset of subjects who screened positive on the mPSS were used to assess clinical characteristics. Participants were categorized into three mutually exclusive groups: 1) unintentional overdose(s) (OD), 2) suicidal ideation or suicide attempt(s) (SI/SA), and 3) suicidal ideation or suicide attempt and unintentional overdose (SI/SA/OD). Multinomial logistic regression models were used to determine whether adverse 
childhood experiences (ACE), self-injurious behaviors, substance use history, overdose history, and past year stressful life events were differentially associated with history of OD/SI/SA. All subjects categorized as having an unintentional OD only, reported having ever used cocaine, while only $72 \%$ of SI/SA subjects had ever used cocaine $(\mathrm{p}=0.02)$. Subjects in the unintentional OD group were more likely to have used heroin in the 24-hours preceding their most recent overdose (92.3\%) compared to SI/SA (30.7\%) and SI/SA/OD (75.0 \%). In the multivariable model subjects with history of SI/SA had higher ACE scores and higher mean episodes of SIB than either of the other two participant groups.

Findings from this study support simultaneously screening for suicide and overdose in OUD inpatient treatment settings. Yet, upon further assessment of clinical and behavior characteristics, history of unintentional OD is distinct from SI/SA. Among patients who screen positive for all three outcomes, SI/SA/OD, there appears to be greater clinical severity. Therefore, it is important to evaluate patient history of suicidal ideation, suicide attempt(s), and overdose(s). More thorough evaluation of drugs involved in overdose and history of self-injury may help distinguish future risk and better inform treatment planning. Collectively this information has implications for resource needs, treatment, and prevention. However, additional research is needed to determine whether screening improves provision of services and reduces self-injurious behavior. 


\section{Dedication}

"The sun is gone, but I have light."

-Kurt Cobain

This dissertation is dedicated to the memory of Richard Anthony Piacquadio. A loving brother, father, son, and friend. He was taken from us too soon, but his light still shines brightly. He lives forever in our hearts. I carried his memorable smile with me throughout this journey and his memory in my heart with me every step of the way. You are loved and missed.

For Beth Anne Picard, an amazing daughter, sister, aunt, and friend. I will always remember your amazing spirit. Not a day goes by that I haven't thought of you. I wish you could be here to celebrate this final accomplishment with me. Your time was too short and left a huge hole in the world that no one can fill. You are loved and missed.

Finally, to all of the patients who took part in this research. For those working on their recovery journey and those who have passed on, you are an inspiration. I am forever grateful. 


\section{Acknowledgements}

I would like to thank Dr. Erin Winstanley, my advisor and dissertation chair for her continued support throughout my graduate studies. I am grateful for her guidance and the mentorship she has provided as I have progressed through my academic career and head into my future as an academic researcher. Thank you also to members of my dissertation committee from the HSOR department: Dr. Kelly, Dr. LeMasters, and Dr. Scott, who have generously given their time and expertise to better my work. I am deeply appreciative of their contribution. To Dr. Ian Rockett and Dr. Gordon Smith, for investing an immense amount of time in my project. I will always remember the many thought-provoking conversations, positivity, and assistance during my time at WVU.

I wish to recognize my colleagues at WVU, including Angie Frame, Mallory Weaver, and Joe Andria for their administrative and their good-natured support. Thank you to Rachel Streets for her guidance while I was on the Dual Diagnosis Unit at Chestnut Ridge, as well as Benjamin Gandee, Mandy Eddy-Spicer, and Leigh Brown at the Center for Hope and Healing. I would also like to thank Brynna Mannibusan and Jen Marton, who helped with many of the administrative tasks that kept my research running, provided endless amounts coffee, and offered much needed emotional support. Both of you are going to do amazing things, and I cannot wait to see how you go forth and set the world on fire! I also wish to extend a special thank you to Dr. James Berry, Dr. Wanghong Zheng, Dr. Dilip Chandran, and Dr. Daniel Farmer for helping to facilitate my research project and patient recruitment within WVU Department of Behavioral Medicine and Psychiatry. Additionally, I want to acknowledge the Biomedical and Behavioral Sciences T32 program run by Dr. Albert Berrebi; thank you for providing me with an amazing 
opportunity to collaborate with so many wonderful student and faculty researchers. Furthermore, I would like to recognize the students of the HSOR program. I am grateful for the many students who shared this experience with me, especially Chibuzo Iloabuchi, Khalid Alhussain, Jayesh Patel, and Richard Sutphin, who were my constant sounding board, my study partners, and who provided me with many fond memories. Thank you for making this a memorable adventure.

I want to acknowledge the many friends who got me through this journey. First, my "hive," you are an amazing group of women; I could not have survived this process without you. To our queen bee, Samantha DeVita for being our source of strength and the glue that holds us together. I would also like to acknowledge Gina Sallinger for her editorial services; you're a "sweet baby angle." Words cannot express how thankful I am for all the support you have provided over the more than 25 years of our friendship; I am forever grateful to share this journey with you. To Hali and Chelsea, you both have been by my side since day one, adopting me as the "third twin." I love you both; I could not have done this without you. TJ Fussell, words cannot express how much your friendship means to me. Thank you for always being the one who will sit with me in the dark; you are one of my greatest sources of strength, I am so lucky to call you my homie. I want to also thank Gloria and Dr. Ang, for reminding me that I am strong. Even when I gave up on myself, you never gave up on me; thank you for always being my strength. Tara Kyser, I know you “can’t relate," but I appreciate your comic relief, words of wisdom, and pictures of Ollie. Thank you for helping me get through this journey; your support carried me through. The Sobeks: Maggie, you have been my biggest fan since the day we met, and Joe cheered me along no questions asked since the day he came into your life. I love you both; thank you for the unwavering support. Last but not least, Jeff, Jacquelyn, Sharon, Shawna, and Trey, I am so grateful to have you in my life. Richard meant the world me and my family, thank you for 
allowing me to dedicate my work to him and letting me honor him in this way and for following my journey. I love you guys!

For my family — the countless aunts, uncles, cousins, and beloved grandparents who made graduate school a possibility and for always believing in my dreams and supporting my quirky, nerdy hobbies. Thank you from the bottom of my heart. Justin, Pauline, and Pete - thank you for being my home away from home in Cincinnati. The three of you made me a part of the family, providing countless night of comfort and support that I greatly needed while pursuing my dreams. I love you all; thank you for being my long-distance cheering section. The DiMuros, who sent me care packages and support messages to keep me going, reminding me that I am always loved and can conquer any challenge, even when I couldn't find the strength to believe in myself. A special thank you to the Sikoras, who attend every function and have become my own personal cheering section. Your love and support mean the world to me.

For my mom, this ant moved the biggest rubber tree plant of all; I made it! I wish you could be here to see all that I have accomplished. You always told me I could be anything, and you were right. I know you are watching over me; I hope you enjoy the view. I miss you every day, but I feel your love every day. This is for you mom! Finally, the biggest thank you of all, my dad and brother. You are the two strongest men I know. Dad, thanks to you I know what it means to work hard and never give up on what you love. I followed my passion because you always pushed me to do what makes me happy. David "Bubby" Stover, you may not know this, but I admire you very much. You work hard and have accomplished so much in your life; you inspire me every day to be my best self. Thank you both for your unconditional love and support; you are my heroes. 


\section{Table of Contents}

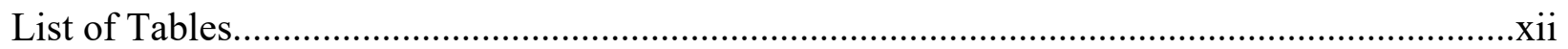

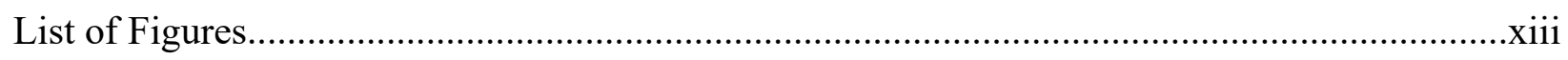

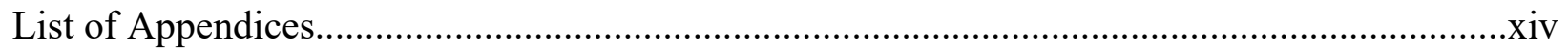

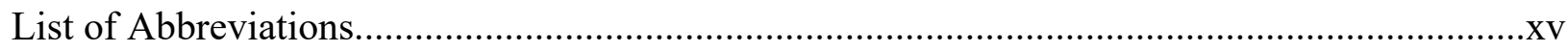

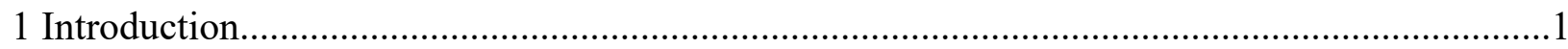

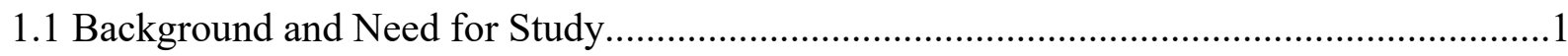

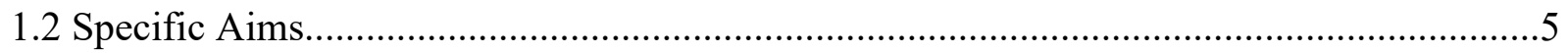

2 Factors Associated with Self-Injurious Behavior, Overdose, and Suicidal Suicide Among Individuals with Opioid Use Disorder: A Systematic Review.........................................

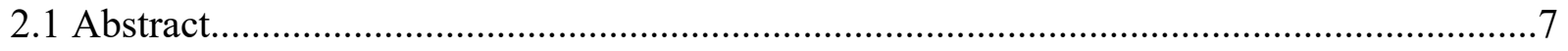

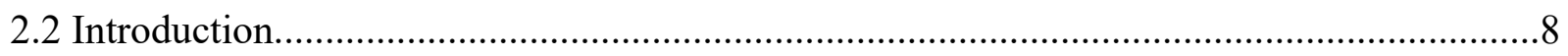

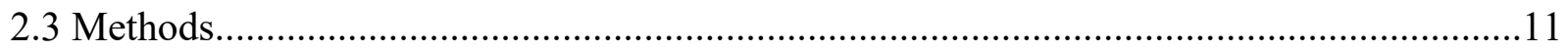

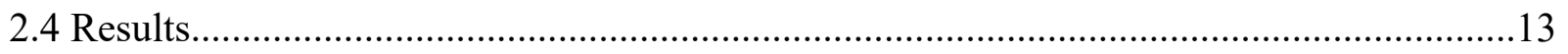

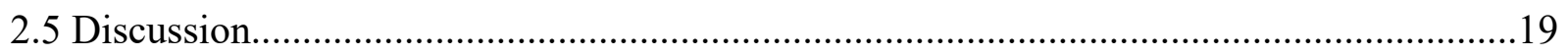

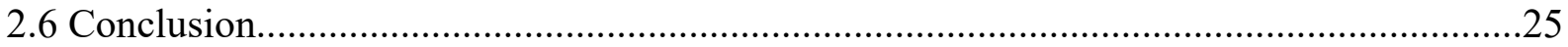

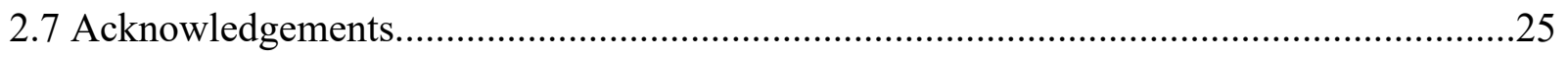

3 The Feasibility and Acceptability of Safety Screening Among Patients Receiving Addiction

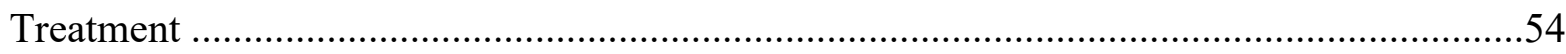

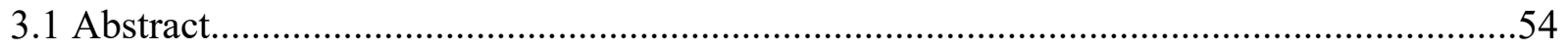

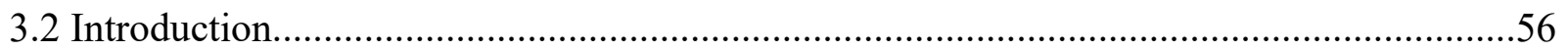

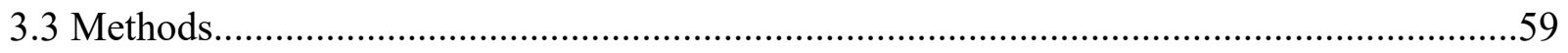

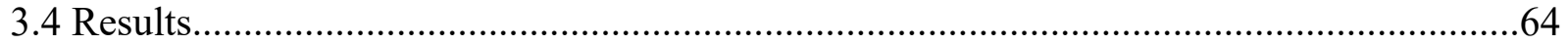




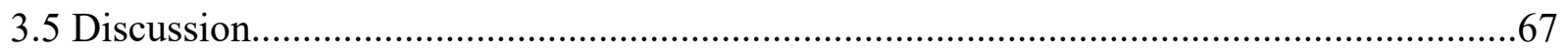

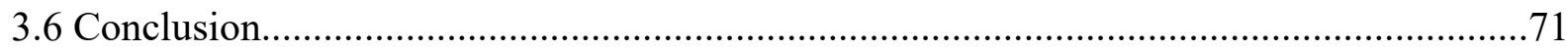

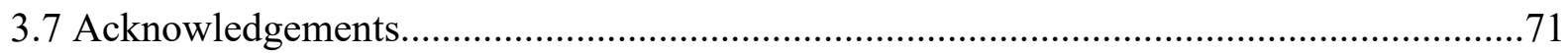

4 Distinguishing Clinical Factors Associated with Unintentional Overdose, Suicidal Ideation, Suicide Attempt, and Opioid Use Disorder.......................................................................

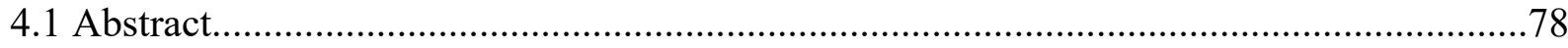

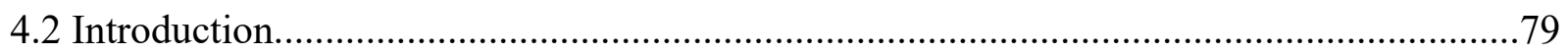

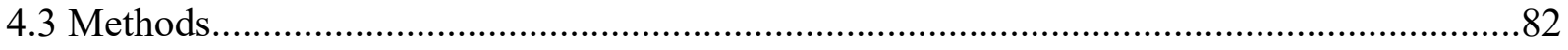

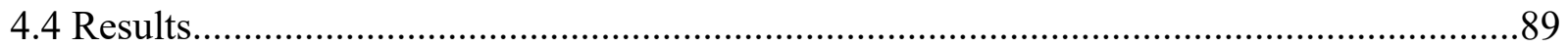

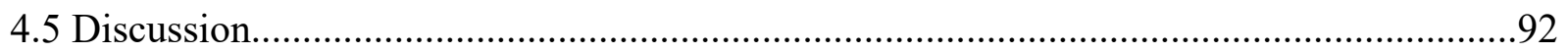

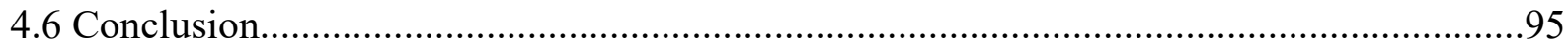

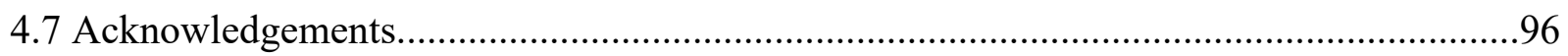

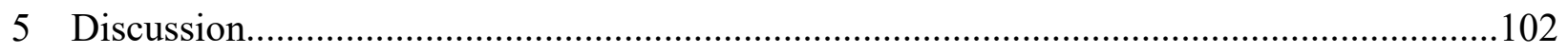

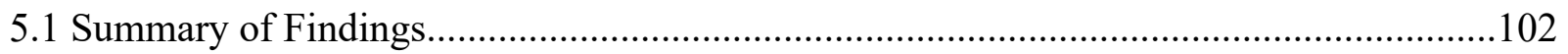

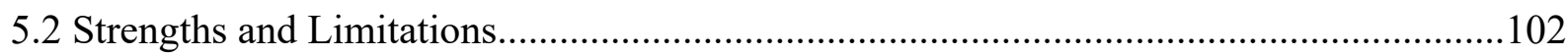

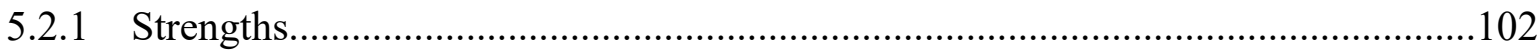

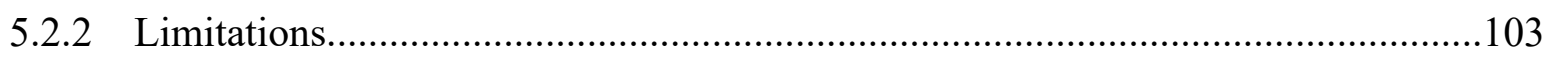

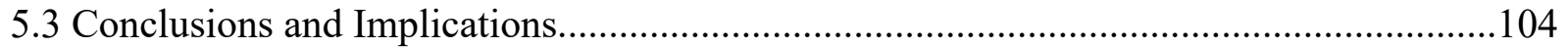

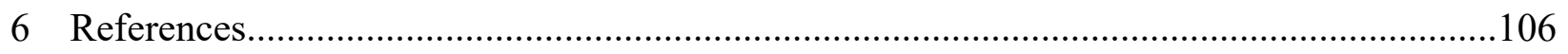

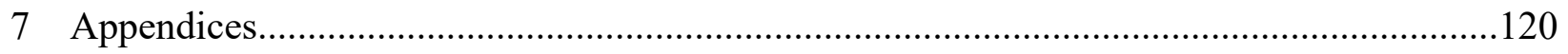




\section{List of Tables}

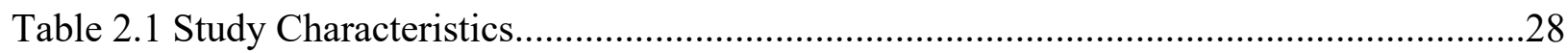

Table 2.2: Quality Assessment and Rating of Systematic Review Studies................................50

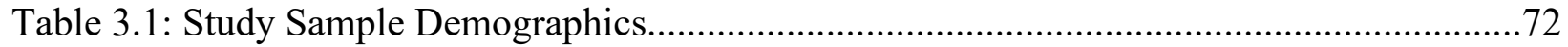

Table 3.2: Modified Patient Safety Screen (mPSS) Responses..............................................73

Table 3.3: Patient Acceptability Questionnaire Responses......................................................74

Table 3.4: Staff Characteristics and Acceptability Questionnaire Responses..........................75

Table 4.1: Overdose History and Substance Use Associated with Recent Overdose..................97

Table 4.2: Adverse Childhood Experiences (ACE) and Life Events Checklist (LEC) Scores.....98

Table 4.3: Measures of Ambivalence and Self-Injurious Behavior (SIB).............................99

Table 4.4: Measures Suicidal Behaviors (Suicidal Ideation and Attempt).............................100

Table 4.5: Multivariable Regression Model....................................................................... 101 


\section{List of Figures}

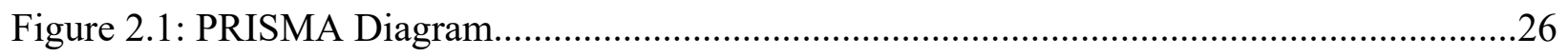

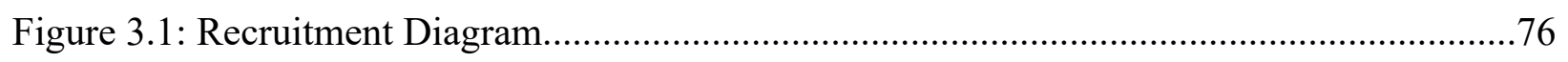

Figure 3.2: Open-ended Response Summary from Staff Acceptability Questionnaire................77 


\section{List of Appendices}

7.1 Supplemental Figure (Chapter 2): Systematic Review Themes......................................120

7.2 Supplemental Figure (Chapter 3): Modified Patient Safety Screen....................................121

7.3 Supplemental Figure (Chapter 3): Visualization of Positive mPSS Screening

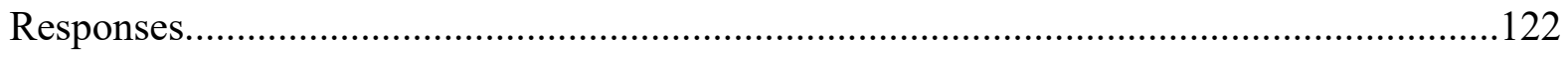

7.4 Supplemental Table (Chapter 4): Expanded Demographic Characteristics of $+\mathrm{mPSS}$

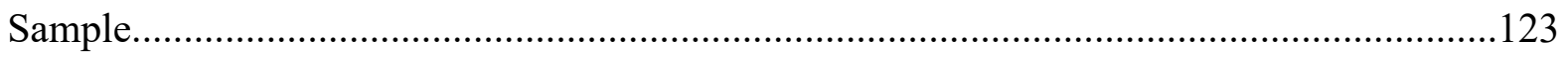

7.5 Supplemental Table (Chapter 4): LEC Individual Item (Not Included in Analysis) .............124 


\section{List of Abbreviations}

\begin{tabular}{|c|c|}
\hline $\mathrm{ACE}$ & Adverse Childhood Experiences \\
\hline AUD & Alcohol Use Disorder \\
\hline $\mathrm{CD}$ & Cannot Determine \\
\hline $\mathrm{CI}$ & Confidence Interval \\
\hline $\mathrm{CL}$ & Checklist Item \\
\hline EMR & Electronic Medical Record \\
\hline IDU & Injection Drug Use \\
\hline LEC & Life Events Checklist \\
\hline mPSS & Modified Patient Safety Screen \\
\hline $\mathrm{N}$ & No \\
\hline NA & Not Applicable \\
\hline NHLBI & National Heart Lung Blood Institute \\
\hline NR & Not Reported \\
\hline OD & Overdose \\
\hline OUD & Opioid Use Disorder \\
\hline PRISMA & Preferred Reporting Items for Systematic Reviews and Meta-Analyses \\
\hline PROMIS & Patient Reported Outcomes Measurement Information System \\
\hline PSS & Patient Safety Scree \\
\hline PTSD & Post-Traumatic Stress Disorder \\
\hline SA & Suicide Attempt \\
\hline SBQ-R & Suicide Behavior Questionnaire-Revised \\
\hline SD & Standard Deviation \\
\hline SI & Suicidal Ideation \\
\hline SIB & Self-Injurious Behavior \\
\hline SITBI-SF & Self-Injurious Thoughts and Behaviors Interview-Short Form \\
\hline SMR & Standardized Mortality Ratio \\
\hline SUD & Substance Use Disorder \\
\hline US & United States \\
\hline WV & West Virginia \\
\hline Y & Yes \\
\hline
\end{tabular}




\section{Chapter 1}

\section{Introduction}

\subsection{Background and Need for Study}

Premature mortality associated with opioid-related overdose and suicide is a significant public health problem in the United States. In 2017, there were 47,600 opioid-related overdoses (14.9 per 100,000) and 47,173 (14.0 per 100,000) suicides reported in the U.S. (Hedegaard et al., 2018; Scholl et al.,2019; SAMHSA, 2019). Previous literature has found that people with OUD have a nearly fifteen times greater risk of all-cause mortality than demographically matched controls, with overdose being the leading cause of death (Degenhardt, et al., 2010). However, for every overdose resulting in death, there are many more nonfatal overdoses. Likewise, having one overdose substantially increases the risk of repeat overdose (Larochelle et al., 2016; Hasegawa et al., 2014) and markedly elevates short-term risks of mortality (Kelty and Hulse, 2017; Stoove et al., 2009). One study, in a cohort of Austrian opioid users, found that following an overdose, people with OUD were nearly 50 times more likely to die than matched community controls, with most of the deaths involving an opioid (Risser et al., 2001). However, disentangling the complex relationship between intentional and unintentional overdoses, even among those overdoses that are nonfatal, remains difficult. In this context, repeated overdoses could be further complicated by history of self-injury, mental health diagnoses, and other life stressors.

Understanding the relationship between overdose and suicide may best be explained by examining key behaviors that precede these events. Approximately $50-60 \%$ of people who die by suicide have a documented prior history of self-injurious behavior (SIB) (Foster et al., 1997) with many instances of SIB occurring shortly before their fatal act (Gairin et al., 2003). 
However, a majority of research studies conducted on SIB utilize adolescent samples, with far fewer studies focusing on adult populations under the assumption that these behaviors decrease in adulthood (Plener et al., 2016). Yet, previous research found individuals with SUDs have a $10-46 \%$ prevalence of SIB compared to $2-4 \%$ reported for the general population (Cummings et al., 2006; Evren \& Evren, 2005; Harned et al., 2010; Maloney et al., 2010). Episodes of SIB are one of the most important risk factors for future suicide (Cavanagh et al.,2003). Still, limited information is available, specifically with regard SIB and future risk for death by suicide, particularly with regard to the prevalence and relationship of lifetime SIB (O'Connor et al., 2016).

The relationship between overdose, suicide, and SIB is further complicated by cooccurring mental health diagnoses. In 2018, individuals with any mental health diagnosis are substantially more likely to misuse opioids in the past year than individuals without any comorbid mental health diagnosis (SAMSHA, 2019). According to the 2018 National Survey of Drug Use and Health, adults with a diagnosis of any mental illness were $9.2 \%$ more likely to have past year opioid misuse, and adults with a serious mental illness were $14.6 \%$ more likely to have past year opioid misuse; as compared to the $2.6 \%$ of the US population over 18 years old with past year opioid misuse and no co-occurring mental health diagnosis (SAMSHA, 2019). Studies consistently reported that depression scores were consistently higher, or history of major depressive episode was present among individuals with OUD for all outcomes of interest (i.e. SIB, suicidal ideation, suicide attempt). Commonly associated co-occurring psychiatric diagnoses reported for patients with SIB, suicidal ideation, and attempt included anxiety, PTSD, and borderline personality disorder. Collectively, these findings support greater psychiatric comorbidity with SIB and suicide attempt among those with OUD. 
Likewise, prior to onset of attempted suicide many individuals with OUD reported significant life experiences. A study by Darke and Ross (2001) found that $82 \%$ of patients with OUD reported that a major life event had preceded their most recent suicide attempt. Research has found that the most commonly reported life events, prior to a suicide attempt among clients with OUD, was the conflict or dissolution of a primary relationship (Darke \& Ross, 2001; Heale et al. 2003), bereavement (Darke \& Ross, 2001; Heale et al. 2003), and impending incarceration (Darke \& Ross, 2001). This finding highlights the complexity of the social dynamics; reinforcing that maintaining social relationships and a support system is key in attaining positive outcomes.

Overlap in SIB and suicide methods is important because SIB is associated with increased risk of a suicide attempt. Previous research has found that cutting is a common form of both SIB and suicide among individuals with OUD. One study found that cutting primary form of SIB for $25 \%$ of treatment seeking OUD patients (de los Cobos et al., 2007). A second study found that $74 \%$ of study participants with OUD engaged in cutting as their predominant form of SIB (Oyefeso et al., 2008). Additionally, three studies found cutting to be in the top three most common means of suicide attempt among individuals with OUD (Conner et al., 2007; Kazour et al., 2016; Neale, 2000). Although it is unknown if SIB is systematically assessed in OUD treatment, this overlap provides insight for means reduction and a identifies a potential point for early intervention. Additionally, while a majority of studies utilized participants who primarily used heroin, however, heroin overdose was not a common method of suicide attempt (Vingoe et al., 1999). Likewise, although heroin was most frequently reported in overdoses, it was significantly more likely to be used in an accidental or unintentional overdose than an intentional overdose (Maloney et al., 2007; Neale, 2000). This is unclear whether this is due to lack of validated instruments to differentiate intentionality of overdose or if this is a result of assessment 
bias. However, the most frequently cited methods of suicide attempt were overdose of nonopioid pills (Conner et al., 2007; Maloney et al., 2007), followed by cutting and hanging (Conner et al., 2007). Overall, firearms were the least likely to be used in attempted suicide among individuals with OUD, contrary to statistics normally reported on the US population. Although a study of veterans suggests firearms are the most common method used in a suicide attempt, they were more commonly used among individuals without a SUD diagnosis (Bohnert et al., 2017). This same study found that poisoning was the most common method of attempted suicide among individuals with a current SUD diagnosis (Bohnert et al., 2017). In 2017, firearms were the most common method of death by suicide, accounting for $50.6 \%$ of all suicide deaths (Kochanek et al., 2017), versus $13.9 \%$ for poisoning (Kochanek et al., 2017).

Overall, the relationship between self-injurious behaviors, suicidal ideation, suicide planning, and suicide attempt has been investigated to varying degrees throughout the published literature. However, there is still a lack of prospective longitudinal data that provide adequate temporal information between onset of OUD and SIB and/or suicide outcomes. Moreover, the relationship between SIB, suicidality, and overdose remains under-researched. In general, patients with OUD at highest risk of attempted suicide and SIB tended to have more severe psychiatric profiles, have increased rates of poly-drug use, experienced significant life stressors and childhood maltreatment. These findings provide critical information for treatment paradigms, including points for intervention and increased services. In order to address the complex nature of overlapping SIB, suicidality, and overdose among individuals with OUD this study has proposed three specific aims to further elucidate this relationship. 


\subsection{Specific Aims}

Specific Aim 1: Conduct a systematic literature review of self-harm behaviors among people with illicit opioid use or opioid use disorder.

Specific Aim 2: Assess the feasibility and acceptability of a standardized screen to measure selfharm and suicide for individuals in OUD treatment.

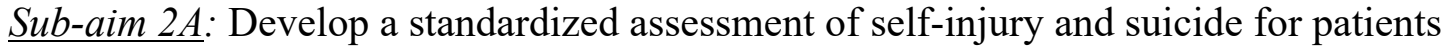
receiving addiction treatment services.

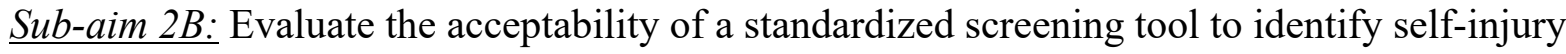
and suicide risk in addiction treatment facilities.

Hypothesis 1: For patients, if the time burden is low and understandability is high, we anticipate that their willingness to complete the assessment will be high.

Hypothesis 2: For clinical staff, if screening does not impose increased clinical burden and can provided information perceived as useful their willingness to integrate the screening tool into clinical practice will be more likely than if perceived burden to clinical staff if high.

Specific Aim 3: Assessment of clinical profiles for three patient groups: 1) unintentional overdose only, 2) suicidal ideation and suicide attempt (SI/SA), and 3) suicidal ideation, suicide attempt, and unintentional overdose (SI/SA/OD). In order to determine group differences multinomial logistic regression models will be employed to evaluate the relationship between groups with age of onset of substance use, age of initiation of SIB, last episode of self-injury, ACE domain scores, and past year stressful life experiences for each of the three groups.

Hypothesis 1: Previous research has related ACEs to earlier age of initiating opioid use, recent injection drug use, and lifetime overdose (Stein et al., 2017). Likewise, studies have reported that accumulation of ACEs increases the odds of both suicidal ideation and 
suicide attempt. Based on previous research, it is believed that perceived stressful life events will be associated with SI/SA while ACE domain scores will be an overlapping factor in both SI/SA (Chen et al., 2009) and the unintentional overdose groups (Stein et al., 2017).

Hypothesis 2: Furthermore, subjects with more recent episodes of SIB will represent the group with a more severe behavioral profile (i.e SI/SA/OD) who engage in more highrisk behaviors and require higher levels of care. 


\section{Chapter 2}

\section{Factors Associated with Self-Injurious Behavior, Overdose, and Suicide Among Individuals with Opioid Use Disorder: A Systematic Review}

\subsection{Abstract}

Background: Both suicide and opioid use disorder (OUD) represent significant threats to public health. In 2018, more than 1.9 million US adults 18 years and older had an OUD; while 1.4 million adults attempted suicide that same year. The key to understanding this relationship is examining behaviors that often precede these potentially fatal acts, such as self-injurious behavior (SIB). The overall objective of this systematic review is to better understand the relationship between SIB, overdose and/or suicidal behaviors among people with OUD. Methods: A systematic literature review was conducted of research studies that examined overdose, SIB, suicide, and OUD. The literature search was conducted in accordance with PRISMA guidelines for systematic reviews and meta-analysis.

Results: Forty-seven studies were included that examined the relationship between overdose, SIB, and/or suicidal behavior among people with OUD. Of these studies 46 were assessed using the NHLBI quality tool, of which $17.4 \%$ were rated as poor, $32.6 \%$ were rated as fair, and $50.0 \%$ of studies were rated as good. Seven common themes were identified including: patterns of substance use, psychological factors/diagnoses, sociodemographic characteristics, family history, traumatic life events, sex, and methods of suicide attempt.

Conclusions: In general, patients with OUD who are at highest risk of suicide attempt and SIB have more severe psychiatric profiles, are female, have increased rates of poly-drug use, and experienced significant life stressors and childhood maltreatment. Collectively this information has implications for resource needs, treatment, and prevention. 


\subsection{Introduction}

Both suicide and opioid use disorder (OUD) represent significant threats to public health. In 2018, more than 1.9 million adults 18 years and older in the United States (US) had an OUD. That same year, 1.4 million adults ages 18 or older attempted suicide (SAMHSA, 2019). However, a key to understanding the relationship between these two conditions is to examine key behaviors that precede these potentially fatal behaviors. Approximately $50-60 \%$ of people who die by suicide have a documented prior history of self-injurious behavior (SIB) (Foster et al., 1997) with many instances of SIB occurring shortly before their fatal act (Gairin et al., 2003). Yet, limited information is available with regard SIB and future risk for death by suicide, specifically, in terms of the prevalence and relationship with lifetime SIB (O'Connor et al., 2018). To date, four articles have assessed the published literature in a systematic review of the relationship between substance use disorders (SUDs) and suicide (Bohnert et al., 2010; Colledge et al., 2020; Harris \& Barraclough, 1997; Wilcox et al., 2004). To varying degrees, each has expanded upon the connection between SUDs and mental health disorders. However, until the recent meta-analysis published by Colledge and colleagues (2020), no review has systematically investigated precursors of suicide and SUDs or a specific SUD (other than alcohol use disorder) and risk factors highly correlated with suicide. Specifically, Colledge colleagues explore the relationship between depression, post-traumatic stress and injection drug use (IDU) (2020). Findings from their analysis indicate that adding suicide prevention to harm-reduction services should be a considered priority based on the strong relationship between self-harm, depression, and IDU (Colledge et al., 2020). Intentionality of overdose is often hard to ascertain and is an outcome that can blur the lines between SIB and suicide attempts. In order to better understand 
the relationship between SIB and/or risk of suicide among people with OUD, a systematic review of published literature was conducted to gauge the current body of literature.

Self-injurious behavior (SIB) is defined as the deliberate destruction of body tissue without suicidal intent, for purposes not deemed socially acceptable. Included are such behaviors as cutting, burning, head banging, picking at wounds, and self-biting (ISSS, 2007). SIB and suicide attempts are linked to increased risk of death by suicide (Cooper et al., 2005; Hawton et al., 2012; O’Connor \& Nock, 2014; O’Conner et al., 2016; Turecki \& Brent, 2016). A majority of research studies conducted on SIB utilize adolescent samples, with far fewer studies focusing on adult populations under the assumption that these behaviors decrease in adulthood (Plener et al., 2016). However, an early study conducted by Briere and Gill (1998) using a stratified, random sample of adults estimated the 6-month prevalence of SIB in adults to be $4 \%$ in the United States (US). Although substantially less attention is paid to co-occurring SIB and SUDs, rates of these behaviors are also elevated in this population. Previous research found individuals with SUDs have a $10-46 \%$ prevalence of SIB compared to $2-4 \%$ reported for the general population (Evren \& Evren, 2005; Harned et al., 2010; Maloney et al., 2010). Episodes of SIB are one of the most important risk factors for future suicide (Cavanagh et al.,2003). When specifically looking at people with OUD, previous research has found these individuals have a standardized mortality ratio approximately 14 times greater than those without OUD (Darke \& Ross, 2002; Harris \& Barraclough, 1997; Tremeau et al., 2008; Wilcox et al., 2004).

A previous meta-analysis by Harris and Barraclough (1997) broadly examined the relationship between 44 mental health diagnoses and their standardized mortality ratio (SMR) for suicide. The overall analysis found that 36 of these diagnoses had excess SMRs relative to the overall population. Specifically, they found that substance abuse was associated with two-fold 
higher risk of mortality when compared to other mental health diagnoses (Harris \& Barraclough, 1997). In 2004, Wilcox and colleagues expanded upon the previous work of Harris and Barraclough in estimating SMRs and SMRs stratified by sex. Their updated review also included evaluation of subcategories of SUDs focusing on alcohol use disorder (AUD), OUD, intravenous drug use (IDU), mixed drug use, and heavy drinking (Wilcox et al., 2004). However, this review did not account for behavioral precursors to suicide or behaviors highly correlated with suicide. Wilcox and colleagues (2004) make a point in their review to address the dearth of literature on AUD and suicide, distinguishing that only a somewhat moderate amount of attention is paid to OUD and suicide. They concluded that limited prospective data on the use of other specific substances of abuse and associations with suicidal behavior manifests in published research. A 2010 review assessed the overlap and risk factors of unintentional overdose and suicide among substance users (Bohnert et al., 2010) and identified substance use as a risk factor for suicide, suicide attempts, and both fatal and non-fatal overdoses.

Previous literature has found that people with OUD have a nearly fifteen times greater risk of all-cause mortality than demographically matched controls, with overdose being the leading cause of death (Degenhardt, et al., 2010). In the US, 70,237 people died from overdose in 2017 (Hedegaard et al., 2018). Of these deaths, opioids were responsible for 47,600 (67.8\%) (Hedegaard et al., 2018). However, for every overdose resulting in death, there are many more nonfatal overdoses. Likewise, having one overdose substantially increases the risk of repeat overdose (Larochelle et al., 2016; Hasegawa et al., 2014) and markedly elevates short-term risks of mortality (Kelty and Hulse, 2017; Stoove et al., 2009). One study, in a cohort of Austrian opioid users, found that following an overdose, people with OUD were nearly 50 times more likely to die than matched community controls, with most of the deaths involving an opioid 
(Risser et al., 2001). However, disentangling the complex relationship between intentional and unintentional overdoses, even among those overdoses that are nonfatal, remains difficult. In this context, repeated overdoses could straddle the line of SIB and suicide attempt(s).

The overall objective of this systematic review is to improve our understanding of relationship between self-injurious behavior (SIB), overdose and/or suicidal behaviors (i.e. suicidal ideation, suicide planning, and suicide attempt) among people with OUD. To achieve this end, the systematic review has two aims: (1) to examine the association between a history of SIB, overdose and/or suicidal behavior in people with OUD and (2) to examine the potential overlapping factors and clinical implications of these behaviors among people with OUD. Thus, the innovation of this review is the aim to address behaviors associated with increased risk for suicidality and OUD to more effectively potential risks and treatment implications associated in populations with multiple morbidities. Furthermore, this review addresses the overlap of overdose with SIB and suicidality, which hitherto has not been explored. Finally, a thematic analysis of research evidence, limitations, and future directions will be discussed.

\subsection{Methods}

\section{Search Strategy}

The literature search followed PRISMA guidelines for systematic reviews and meta-analysis (Moher et al., 2009). Articles were retrieved through a systematic search of electronic peerreview literature across three databases: the U.S. National Library of Medicine's PubMed electronic database, the American Psychological Association's PsycINFO electronic database, and the Institute for Scientific Information's Web of Science electronic database. All databases were searched for articles published between January 1, 1998 and August 31, 2018. A 20-year timeframe was selected to overlap intentionally with the review published by Bohnert and 
colleagues (2010), and to capture new, relevant studies on this topic and to expand to include literature addressing SIB.

Each database had a slightly different search structure. To accommodate varying search parameters, terms were coupled with relevant MeSH/thesaurus terms and truncated, with spelling variations as appropriate. A combination of the following terms "self-harm," "self- injury," "deliberate self-harm," "self-directed violence," "self-injurious behavior," "non-suicidal selfharm," "suicide," “opioid abuse," "opioid misuse," "heroin abuse," "narcotics abuse," "opiate abuse," "opiate misuse," "opiate use disorder," and "opioid use disorder" were used within the search parameters for article retrieval.

Studies were limited to research articles published in peer-reviewed journals available in English. The search results were imported into a bibliographic software program (EndNote X9), and duplicated articles were removed. Articles were excluded if they: (1) failed to provide new data (e.g., review articles), (2) data were limited to case reports or only descriptive in nature, (3) their sample was limited to children (i.e., the sample age cutoff was $<18$ years), (4) their sample excluded persons who used opioids or had a sub-group of participants with opioid use, abuse, or OUD (i.e., clustered substances of abuse together), (5) they did not examine SIB and/or suicide and opioid use/OUD, (6) the study examined both SIB and/or suicide and opioid use/OUD but did not test for an association between these outcomes (i.e., did not provide data on outcomes relative to one another beyond prevalence), and/or (7) the study was conducted using toxicology or post-mortem data only. Titles and abstracts were independently screened, and then full texts were reviewed to identify eligible studies. Reference lists from eligible research articles were searched for further studies that might be included in the final review. Any disagreements in article inclusion were resolved by discussion or between the co-authors. 


\section{Quality Assessment}

The quality of studies was examined using the National Heart, Lung, and Blood Institute (NHLBI) quality assessment tool (2014). This tool was chosen because it evaluates quality based on study design. The tool employs one check list tailored for observational cohort and crosssectional studies and another checklist for case-control studies. Both checklists were developed to address internal validity (i.e., risk of bias) in an equivalent manner. Each checklist item was rated as "yes," "no," "not applicable (NA)," "cannot determine (CD)," or "not reported (NR)." These items then inform an overall quality rating for each study as "good," "fair," or "poor."

\subsection{Results}

The PubMed search resulted in 672 articles, the PsycINFO search identified 970 articles, and the Web of Science search yielded 972 articles. After deletion of duplicate articles, a total of 1,631 unique articles remained. Following an initial article inspection (i.e. title and keywords), 143 abstracts were reviewed and a total of 47 articles remained that were relevant to this systematic review (Figure 2.1).

Twenty-six of the 47 studies examined more than one outcome. Ten studies examined overdose and suicide attempt (Best et al., 2000; Bradvik et al., 2007; Conner et al., 2007; Darke \& Ross, 2001; Heale et al., 2003; Neale, 2000; Ross et al., 2005; Rossow \& Lauritzen, 1999; Ravndal \& Vaglum, 1999; Vingoe et al., 1999), one additional study examined overdose with SIB and suicide attempts (de los Cabos et al., 2007), and another included all drug poisonings (Olsson et al., 2016). Five studies reported on suicidal ideation and suicide attempt (Cheek et al., 2016; Kazour et al., 2016; Kuramoto et al., 2012; Maloney et al., 2009; Wines et al., 2004). Four studies assessed suicide planning, in addition to suicidal ideation and suicide attempt (Ashrafioun et al., 2017; Darke et al., 2015; Havens et al., 2004; Marchand et al., 2017). Five 
studies examined SIB and suicide attempt (Baykara \& Alban, 2018; Darke et al., 2010; Darke et al., 2012; Harned et al., 2006; Maloney et al., 2010). One study examined suicide attempt, overall suicide risk, fatal accidental intoxication, and injury/intoxication of undetermined intent (Olsson et al., 2016) (Table 2.1).

Quality of Studies

Forty-six studies in the present review used quantitative methods that could be evaluated using the NHLBI quality assessment tool. One study employed a mixed methods design and primarily reported qualitative data (Neale, 2000). Hence it was not assessed for quality. Of the studies assessed using the NHLBI quality tool, $17.4 \%$ had an overall rating of poor, $32.6 \%$ were rated as fair, and $50.0 \%$ good. Forty-two of the studies relied on self-report measures, $66.7 \%$ of which did not use validated measure for suicide, overdose, or SIB outcomes. Recall bias was the predominant research limitation across all studies included in the review. Seventy-two percent of studies use statistical modeling to address potential confounding by controlling for known covariates associated with the outcome of interest. However, only $21.7 \%(n=10)$ of the studies provided calculations for sample or effect sizes (Table 2.2).

Themes

\section{Patterns of Substance Use}

Twenty-six studies reported on patterns of substance abuse associated with SIB and suicidal behaviors. Four studies analyzed the relationship between SIB and OUD. Of these studies, three found that SIB was associated with increased likelihood of having OUD (Darke et al., 2010, Lavania et al., 2012, \& Maloney et al., 2010). Two of these studies also found an increased rate of AUD and OUD (Darke et al., 2010 and Maloney et al., 2010). One study found that patients with a dually diagnosed AUD and OUD were more likely to report SIB. However, they were less 
likely to report suicide attempts than participants with other SUDs (Darke et al., 2010). Five studies assessed the relationship between suicidal ideation and OUD. Three studies found that OUD was associated with a statistically increased likelihood of suicidal ideation (Ashrafioun et al., 2017, Kuramoto et al., 2012, \& Jin et al., 2013), specifically among samples with individuals who predominantly used heroin.

Eighteen examined suicide attempts or risk and OUD. The most common overlapping risk factor between OUD and suicide attempt(s)/risk of suicide was poly-drug use (Darke \& Ross, 2001; Darke et al., 2004; Darke et al., 2015; Hakansson et al., 2010; Harned et al., 2006; Havens et al., 2004; Rossow \& Lauritzen, 1999). Additionally, four studies found the likelihood of recent heroin overdose (Darke et al., 2004), history of overdose (Hankansson et al., 2010), and history of a suicide attempt increased with the number of previously reported heroin overdoses (Bradvik et al., 2007; Rossow \& Lauritzen, 1999).

\section{Psychological Factors/Diagnoses}

Twenty-four studies reported specific psychological symptom and /or psychiatric diagnoses associated with suicidal behaviors, SIB, and OUD. Fifty-four percent of studies reported an association with suicidal behaviors and/or SIB and major depressive disorder or depressive episodes. Two studies found that study participants who met the criteria for both OUD and SIB also were more likely to have met the criteria for a depressive episode (Maloney et al., 2010) and had significantly higher scores on the Beck Depression Scale than patients without SIB (Baykara \& Alban, 2018). In addition, one study found that treatment with antidepressant medication was a significant predator of suicide attempt among those with OUD (Roy, 2010). Two studies found that participants with OUD, suicide attempts, and SIB were more likely to have been diagnosed with borderline personality disorder (Darke et al., 2004; 
Maloney et al., 2010). Another frequently reported psychiatric diagnosis was posttraumatic stress disorder (PTSD). Two studies found that a recent suicide attempt among participants with OUD was associated with a diagnosis of PTSD (Darke et al., 2004; Darke et al., 2015). One of these studies found PTSD to be significantly associated with SIB among those with OUD (Darke et al., 2004). Three additional studies reported overall increased reporting of overall history of mental health problems: one specifically with regards to intentional overdose (Neale, 2000), another reported higher odds of suicidal ideation (Marchand et al., 2017), and a third found that patients with a history of both a suicide attempt and overdose had increased psychiatric problemsspecifically anxiety, depression, and suicidal ideation (Rossow \& Lauritzen, 1999).

\section{Family History}

Six studies investigated the association between family history of suicidal behaviors and suicidal behaviors, SIB, and OUD. Two studies found that people with OUD were more likely to report a history of suicide attempts among family members (Dragisic et al., 2015; \& Roy, 2002). One more found that family history of suicidal behavior predicted a suicide attempt in people with OUD (Roy, 2010). Additionally, another study found that maternal alcohol use or psychiatric problems and paternal alcohol problems were significantly associated with a suicide attempt in patients with OUD (Hakansson et al., 2010).

\section{Role of Gender}

Fifteen studies investigated the role of gender and suicidal behaviors or SIB and OUD. Three studies found that females with OUD were significantly more likely to report a history of SIB than males (Darke et al., 2010; Darke et al., 2012; Maloney et al., 2010). Seven reported an association between gender and a suicide attempt among participants with OUD. Similar to the studies that examined this relationship to SIB, females with OUD were significantly more likely 
to have a history of a suicide attempt(s) than males (Bohnert et al., 2017; Hakansson et al., 2010; Kazour et al., 2016). Females with OUD who attempted suicide also were more likely to be younger than those with no such history (Dark et al., 2012; Roy 2012), attempt suicide more frequently than males (Darke et al., 2001; Darke et al., 2015; Maloney et al., 2007; Tremeau et al., 2005), and use less lethal methods (Icick et al., 2017). Two studies found that females were more likely than males to have attempted suicide prior to the onset of heroin use (Darke \& Ross, 2001; Maloney et al., 2007), with one finding that males were more likely than females to be using heroin at the time of their most recent suicide attempt (Darke \& Ross, 2001).

\section{Sociodemographic Factors}

Ten studies examined sociodemographic characteristics associated with the relationship between suicidal behaviors, SIB, and OUD. Four studies investigated age at time of suicide attempt and/or SIB. Overall, younger OUD patients were more likely than older counterparts to have attempted suicide (Baykara \& Alban, 2018; Roy 2010). Consistent with previous research, OUB patients engaged in SIB at a significantly younger than their age of a first suicide attempt (Darke et al., 2012), primarily beginning in adolescence (Maloney et al., 2010). Conversely, another study reported that risk of suicide attempt was associated with older age among patients with OUD (Hakansson et al., 2010). Two studies found that suicidal ideation was significantly more common among OUD patients who lived alone (Darke et al., 2015; Maremmani et al., 2007). Similarly, overdoses were reported more frequently among people who were homeless, had only drug-using friends, or no friends at all (Rossow \& Lauritzen,1999). Marital status, specifically being unmarried or divorced was also significantly associated with an increased risk of suicide attempt/suicidal ideation and OUD (Darke et al., 2015; Dragisic et al., 2015; Jin et al., 2013). Additionally, two of the studies found that participants with OUD, who more frequently 
reported suicidal ideation and suicide attempts, were significantly more likely than those who reported less frequency to be unemployed (Darke et al., 2015; Maremanni et al., 2007).

\section{Childhood and Adulthood Life Events}

Eleven studies evaluated the contribution of both childhood and adult life events and their association with suicidal behaviors and SIB among patients with OUD. Participants with OUD who experienced childhood maltreatment/trauma (i.e. emotional abuse, physical abuse, sexual abuse, emotional neglect, and physical neglect) were more likely to report a history of suicide attempt(s) (Kalyoncu et al., 2007; Maloney et al., 2007; Maloney et al., 2010; Roy 2002). One study reported that for each unit increase in the childhood emotional neglect score, the adjusted odds of suicidal ideation increased by nine percent (Marchand et al., 2017). Participants with OUD and a self-reported history of SIB were also more likely to report every subtype of childhood maltreatment, particularly sexual abuse and neglect (Maloney et al., 2007). Individuals who reported experiencing a violent physical assault, before age sixteen were significantly more likely to self-report a history of SIB and a suicide attempt (Darke et al., 2012). Furthermore, participants with OUD and prior sexual assault were more likely to report severe suicidal ideation (Gilmore et al., 2008). History of emotional, physical, and sexual abuse was also positively and independently associated with a history of attempted suicide (Hakansson et al., 2010). In adulthood, the number of stressful or traumatic life events was significantly associated with that outcome (Chen et al., 2009; Marchand et al., 2017). One study of young IDUs found that the odds of a suicide attempt increased by $22 \%$ with each violent exposure reported (Havens et al., 2004). Similarly, the number of stressful life events experienced by a person was positively associated with self-reported SIB (Lavania et al., 2012).

Triggers for Behavior and Methods of Suicide Attempt 
Eight studies reported on behaviors associated with methods of suicide attempts and/or SIB or triggers for engaging in those behaviors. The most commonly reported method of attempted suicide was overdose using non-opioid pills (Conner et al., 2007; Maloney et al., 2007; Vingoe et al., 1999). In the two studies that did report opioids as the primary method of suicide attempt, the sample was predominately heroin users who attempted suicide via heroin overdose (Kazour et al., 2016; Neale, 2000). The two most commonly reported triggers of attempted suicide were a conflict or dissolution in the primary relationship and bereavement (Darke \& Ross, 2001; Heale et al., 2003).

\subsection{Discussion}

\section{Main Findings}

This systematic review included 47 studies that examined the relationship between overdose, SIB, suicidal ideation, and suicide attempts among patients with OUD. To date, four review articles have reviewed the literature examining the association between substance use disorders (SUDs) and suicide (Bohnert et al., 2010; Colledge et al., 2019; Harris \& Barraclough, 1997; Wilcox et al., 2004). To varying degrees, each has expanded upon this connection; however, until Colledge and colleagues (2019), no review has systematically investigated precursors of suicide and SUDs or at a specific SUD (other than alcohol use disorder) and risk factors highly correlated with suicide. Understanding these precursors of suicidal behavior and the overlapping risk factors between OUD and SIB are key in examining risks for potentially fatal behaviors.

In 2018, individuals with any mental health diagnosis are substantially more likely to misuse opioids in the past year than individuals without any comorbid mental health diagnosis (SAMSHA, 2019). According to the 2018 National Survey of Drug Use and Health, adults with a 
diagnosis of any mental illness were $9.2 \%$ more likely to have past year opioid misuse and adults with a serious mental illness were $14.6 \%$ more likely to have past year opioid misuse; this is compared to the $2.6 \%$ of the US population over 18 years old with past year opioid misuse and no co-occurring mental health diagnosis (SAMSHA, 2019). Consistent with these findings, fiftyone percent of the studies in this review reported associations between psychiatric diagnoses, SIB, and suicidality among individuals with OUD. Studies consistently reported that depression scores are consistently higher, or history of major depressive episode is present among individuals with OUD for all outcomes of interest (i.e. SIB, suicidal ideation, suicide attempt). Commonly associated co-occurring psychiatric diagnoses reported for patients with SIB, suicidal ideation, and attempt included anxiety, PTSD, and borderline personality disorder. Collectively these findings support greater psychiatric comorbidity with SIB and suicide attempt among those with OUD.

Overall, females were more likely to report a history of SIB, suicidal ideation, and suicide attempt, which is similar to that found in the general population (Bohnert et al., 2017; Hakansson et al., 2010; Kazour et al., 2016; Olfson et al., 2017). Females were also more likely to make multiple attempts (Darke et al., 2012; Darke et al., 2015; Tremeau et al., 2005). Females were also more likely to report recent episodes of SIB (past 12-months) despite older age compared to males (Darke et al.,2012), though age of onset of SIB consistently occurred in adolescents and before the first suicide attempt was made for both males and females (Darke et al., 2012; Maloney et al., 2010). One study reported that females with OUD were 2-3x more likely to die by suicide than males (Bohnert et al., 2017). This is contrary to statistics reported among the general population in the US where men are 3.54 times more likely to die by suicide than women (Kochanek et al., 2017). Specifically, with regards to heroin use, it was noted the 
females were more likely to attempt suicide prior to the onset of heroin use compared to males (Maloney et al., 2007), with one study reporting that females were 18 times more likely to attempt suicide before initiation of heroin use compared to males (Darke \& Ross, 2001). These studies suggest that females with OUD are not only at higher risk of suicide attempt, but at higher risk for making multiple attempts when compared to males irrespective of transition to heroin use. This could in part be due to co-occurring risks for other psychiatric diagnoses; however, given higher reporting rates of SIB among females suicide risk remains a significantly greater threat among females.

Nationally representative samples have found that adults in the US reporting recent suicide attempts are more likely to be female, white, unemployed, and have a family history of suicide attempt irrespective of any other comorbid health diagnoses (Olfson et al., 2017). Additionally, being widowed, divorced, or unmarried was also significantly associated with suicide attempt independent of mental health or SUD diagnosis (Olfson et al., 2017). Findings from several studies reporting on demographic characteristics in combination with suicidality among individuals with OUD are consistent with those reported in the general population. Specifically, being unmarried (Dragisic et al., 2015; Jin et al., 2013; Maremmani et al., 2007), unemployed (Darke et al., 2015; Jin et al., 2013), living alone (Darke et al., 2015; Maremmani et al., 2007) and homeless (Rossow \& Lauritzen, 1999) were all significantly correlated with increased history of suicidal ideation. Likewise, one study included in this review also reported that irrespective of diagnosis of OUD patients with history of suicide attempt were more likely to have a family member with a history of suicide attempt (Maloney et al., 2007) which coincides with reports from research done in general samples. Although these factors were not consistently examined across studies included in the review, several consistencies are present in demographic 
characteristics associated with suicidality independent of OUD diagnosis. This overlap in employment status, marital status, and family history can potentially help identify global characteristics of suicidality that remain constant irrespective of diagnosis of OUD.

An important finding from this review is the role of treatment. Although few studies measured the impact of treatment with respect to the outcomes of interest (i.e. SIB, suicidal ideation, and/or suicide attempt); several studies did provide some key points. Patients with OUD in treatment settings who reported suicide attempts generally tended to be in overall poorer health or have chronic medical conditions (Darke et al., 2015; Hakansson et al., 2010; Roy, 2010). Likewise, previous number of times detoxed was positively associated with number of suicide attempts (Kazour et al., 2016). Similarly, patients with deliberate overdoses were more likely to be in treatment at the time of overdose (Best et al., 2000).

Prior to onset of suicide attempt many individuals with OUD reported significant life experiences. A study by Darke and Ross (2001) found that $82 \%$ of patients with OUD reported that a major life event had preceded their most recent suicide attempt. Research has found that the most commonly reported life events prior to suicide attempt among clients with OUD was the breakdown of a primary are conflict or dissolution of a primary relationship (Darke \& Ross, 2001; Heale et al. 2003), bereavement (Darke \& Ross, 2001; Heale et al. 2003), and impending incarceration (Darke \& Ross, 2001). This highlights the complexity of the social dynamics; particularly, as mentioned earlier, that living alone, and poor social functioning are also associated with increased risk for both suicidal ideation and suicide attempt. Maintaining social relationships and having support is key in attaining positive outcomes.

While a majority of studies utilized participants who primarily used heroin, interestingly, heroin overdose was not a common method of suicide attempt (Vingoe et al., 1999). Likewise, 
even though heroin was most frequently reported in overdoses, it was significantly more likely to be used in an accidental overdose than a deliberate overdose (Maloney et al., 2007; Neale, 2000). This is unclear whether this is due to lack of validated instruments to differentiate intentionality of overdose or if this is a result of assessment bias. However, the most frequently cited methods of suicide attempt were overdose of non-opioid pills (Conner et al., 2007; Maloney et al., 2007), followed by cutting and hanging (Conner et al., 2007). Overall, guns were the least likely to be used to attempt suicide among individuals with OUD contrary to statistics normally reported among suicide attempts in US population. Although a research done among veterans suggests that firearms are the most common method used in suicide attempt, firearms were more commonly used among individuals without any SUD diagnosis (Bohnert et al., 2017). This same study found that the most common methodology for suicide attempt among individuals with any current SUD diagnosis was poisoning (Bohnert et al., 2017). In 2017, firearms were the most common method of death by suicide accounting for $50.6 \%$ of all suicide deaths (Kochanek et al., 2017); whereas poisoning accounted for $13.9 \%$ of suicide deaths in the US (Kochanek et al., 2017).

Overlap in SIB and suicide methodology are of importance because SIB is associated with increased risk of suicide attempt. Previous research has found that cutting is a common form of both SIB and suicide among individuals with OUD. One study found that $25 \%$ of treatment seeking OUD patients primary form of SIB was cutting (de los Cobos et al., 2007). A second study found that $74 \%$ of study participants with OUD engaged in cutting as their predominant form of SIB (Oyefeso et al., 2008). Additionally, three studies found cutting to be in the top three most common forms of suicide attempt among individuals with OUD (Conner et al., 2007; Kazour et al., 2016; Neale, 2000). Although it is unknown if SIB is systematically 
assessed in OUD treatment, this overlap provides insight for means reduction and a potential point for early intervention.

\section{$\underline{\text { Implications }}$}

A total of two studies reported an overall increased risk of suicide specifically associated with OUD. One of these studies found that inpatients with heroin use had a 16-fold increase in standardized mortality ratio due to suicide compared to the general population (Pan et al., 2014). A second, reported 1.76 odds of death by nonviolent suicide among individuals with OUD compared to those without OUD (Ilgent et al., 2009). Two indicators of substance use severity were found to be associated with suicide attempt in this review: age of onset of opioid use and IDU. Several studies reported that an earlier onset of substance use was associated with increased risk of suicide attempt among people with OUD (Dragisic et al., 2015; Havens et al., 2004; Icick et al., 2017; \& Kazour et al., 2016). Likewise, IDUs with OUD also had an increased risk for suicide attempt (Dragisic et al., 2015; Hakansson et al., 2010; Masferrer et al., 2016). Both of these factors overlap with risk of suicide attempt and risk for potential overdose marking increased severity of substance use. Furthermore, as summarized by previous studies those who maintain relationships with only friends in the drug use community are further at risk of suicidal behaviors. The connection between these factors indicates that poor social functioning, psychiatric decline, and substance use not only correlate to suicidality but represent key factors for intervention.

\section{$\underline{\text { Limitations }}$}

Twenty-six studies were cross section, thus temporal conclusions could not be drawn linking onset of OUD with SIB, suicidal ideation, and/or suicide attempt. Five studies included longitudinal data limiting the ability to identify true causal relationships between OUD and SIB 
and/or suicidal behaviors due to lack of temporal information. Furthermore, a majority of this research was conducted among inpatient or outpatient treatment samples which limits generalizability. It is also unknown whether treatment seeking individuals have more functional impairments or experience more symptom severity than non-treatment seeking individuals. Likewise, limited studies are conducted in low-income, rural areas which may have different patterns of substance use and barriers to treatment further contributing to the variability in estimation of SIB and suicide among people with OUD. Only one study in this review examined overdose and SIB indicating a need for further investigation of this relationship in future research, especially given the strong connection between SIB and future risk for suicide attempt.

\subsection{Conclusions}

Overall, the relationship between self-injurious behaviors, suicidal ideation, suicide planning, and suicide attempt has been investigated to varying degrees throughout the published literature. However, there is still lack of prospective longitudinal data providing adequate temporal information between onset of OUD and SIB and/or suicide outcomes. Furthermore, the relationship between SIB, suicidality, and overdose still remains under researched. In general, patients with OUD who are at highest risk of suicide attempt and SIB tend to have more severe psychiatric profiles, increased rates of poly-drug use, tend to be female, experience significant life stressors, and childhood maltreatment. These findings provide crucial points for treatment paradigms including points for intervention and increased services to address the complex nature overlapping SIB, suicidality, and OUD.

\subsection{Acknowledgements}

Funding Source: ANS was supported by the National Institute of General Medical Sciences of the National Institutes of Health (1 T32 GM132494). 
Figure 2.1: Prisma Flow Diagram
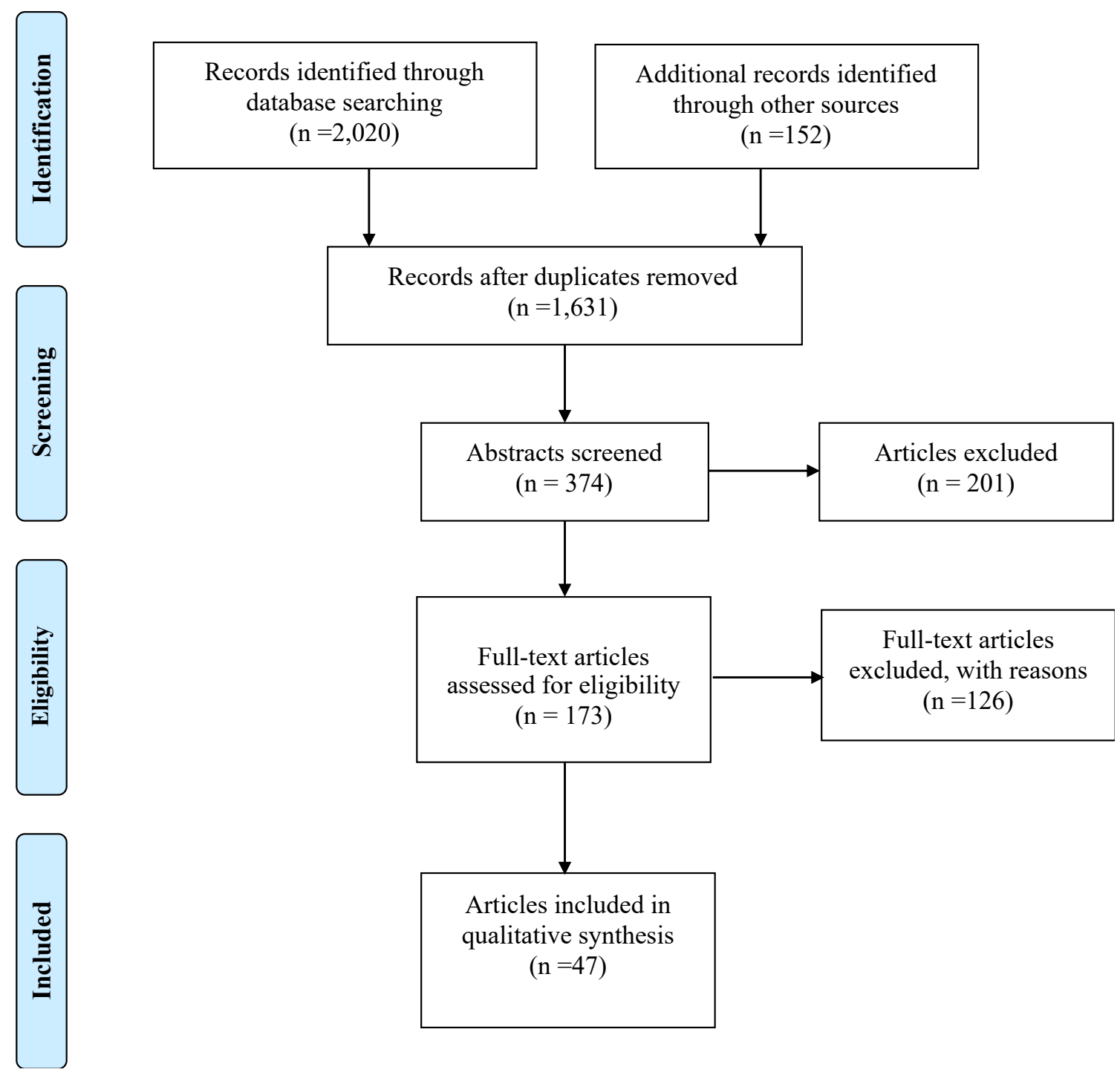


\begin{tabular}{|c|c|c|c|c|c|c|c|}
\hline $\begin{array}{c}\text { Author } \\
\text { (Pub year)/ } \\
\text { Country }\end{array}$ & Study Design & $\begin{array}{l}\text { Overall Sample } \\
\text { (OUD) }\end{array}$ & $\begin{array}{c}\text { Sample } \\
\text { Characteristics }\end{array}$ & $\begin{array}{l}\text { Data Sources \& } \\
\text { Measure(s) }\end{array}$ & $\begin{array}{l}\text { Substances } \\
\text { Examined }\end{array}$ & $\begin{array}{c}\text { Outcomes } \\
\text { (Time frame) }\end{array}$ & Key findings/ Summary \\
\hline $\begin{array}{l}\text { Ashrafioun et al. } \\
(2017) / \text { USA }\end{array}$ & $\begin{array}{l}\text { Cross Sectional } \\
\text { Study }\end{array}$ & $41,053(365)$ & $\begin{array}{l}2014 \text { National } \\
\text { Survey on Drug } \\
\text { Use and Health } \\
\text { (NSDUH). }\end{array}$ & & $\begin{array}{l}\text { Prescription } \\
\text { opioids, alcohol, } \\
\text { hallucinogens, } \\
\text { inhalants, } \\
\text { stimulants, } \\
\text { marijuana, } \\
\text { sedatives, } \\
\text { tranquilizers }\end{array}$ & $\begin{array}{l}\text { Suicide ideation, suicide } \\
\text { planning, SAs (past 12- } \\
\text { months) }\end{array}$ & $\begin{array}{l}\text { - Adjusted models found that } \\
\text { prescription opioid misuse was } \\
\text { significantly associated with SI } \\
\text { for each frequency category. } \\
\text { - Individuals who reported an } \\
\text { average of weekly or more use } \\
\text { had a significant association } \\
\text { with suicidal planning and } \\
\text { attempts. }\end{array}$ \\
\hline $\begin{array}{l}\text { Baykara \& Alban } \\
\text { (2018)/Turkey }\end{array}$ & $\begin{array}{c}\text { Cross Sectional } \\
\text { Study }\end{array}$ & $100(100)$ & $\begin{array}{l}\text { Inpatients between } \\
18-65 \text { yrs old } \\
\text { diagnosed with } \\
\text { OUD. }\end{array}$ & - DSM-V & Opioids & $\begin{array}{l}\text { Self-Injurious Behavior } \\
\text { (SIB), SAs ( } 4-6 \text { weeks } \\
\text { after last use of } \\
\text { substance; lifetime } \\
\text { history) }\end{array}$ & $\begin{array}{l}\text { - Patients with history of OUD \& } \\
\text { SIB had higher subscale scores } \\
\text { for passive aggression, } \\
\text { somatization, and immature } \\
\text { factor. } \\
\text { - Beck depression and Beck } \\
\text { anxiety inventory scores were } \\
\text { higher for patients with both } \\
\text { OUD and history of SA. } \\
\text { - Logistic regression analysis } \\
\text { found that passive aggression } \\
\text { subscale scores and younger } \\
\text { age were related to SIB. } \\
\text { History of SA was associated } \\
\text { with younger age, suppression, } \\
\text { dissociation, somatization, } \\
\text { higher Beck anxiety inventory, } \\
\text { low idealization, projection, } \\
\text { devaluation, splitting, and } \\
\text { rationalization scores. }\end{array}$ \\
\hline $\begin{array}{l}\text { Best et al. } \\
(2000) / \text { UK }\end{array}$ & $\begin{array}{l}\text { Cross Sectional } \\
\text { Study }\end{array}$ & $200(200)$ & $\begin{array}{l}\text { Patients in } \\
\text { methadone } \\
\text { treatment in } \\
\text { Edinburgh and } \\
\text { south London. }\end{array}$ & & Methadone & $\begin{array}{l}\text { Accidental OD; } \\
\text { deliberate OD (lifetime) }\end{array}$ & $\begin{array}{l}\text { - Patients with a deliberate OD } \\
\text { were more likely to currently be } \\
\text { taking diazepam, have higher } \\
\text { depression scores, hopelessness } \\
\text { scores, more lifetime ODs, \& } \\
\text { OD more recently. } \\
\text { - The deliberate OD group was } \\
\text { more likely to be in treatment at } \\
\text { the time of OD and more } \\
\text { frequently reported SI in the } \\
\text { past-month than individuals } \\
\text { who reported accidental OD. }\end{array}$ \\
\hline
\end{tabular}




\begin{tabular}{|c|c|c|c|c|c|c|c|}
\hline $\begin{array}{c}\text { Author } \\
\text { (Pub year)/ } \\
\text { Country }\end{array}$ & Study Design & $\begin{array}{l}\text { Overall Sample } \\
\text { (OUD) }\end{array}$ & $\begin{array}{c}\text { Sample } \\
\text { Characteristics }\end{array}$ & $\begin{array}{l}\text { Data Sources \& } \\
\text { Measure(s) }\end{array}$ & $\begin{array}{l}\text { Substances } \\
\text { Examined }\end{array}$ & $\begin{array}{c}\text { Outcomes } \\
\text { (Time frame) }\end{array}$ & Key findings/ Summary \\
\hline $\begin{array}{l}\text { Bohnert et al. } \\
(2017) / \text { USA }\end{array}$ & $\begin{array}{l}\text { Cross Sectional } \\
\text { Study }\end{array}$ & $\begin{array}{l}4,836,086 \\
(37,908)\end{array}$ & $\begin{array}{l}\text { Veteran Health } \\
\text { Association } \\
\text { (VHA) users from } \\
\text { the } 2005 \text { fiscal } \\
\text { year and alive at } \\
\text { the beginning of } \\
\text { the } 2006 \text { fiscal } \\
\text { year. }\end{array}$ & $\begin{array}{l}\text { - National Death } \\
\text { Index (NDI) \& } \\
\text { ICD-10 (suicide } \\
\text { mortality) } \\
\text { - VHA National } \\
\text { Patient Care } \\
\text { Database (NPCD) } \\
\text { and ICD-9 CM } \\
\text { (SUDs) }\end{array}$ & $\begin{array}{l}\text { Any SUD, AUD, } \\
\text { cocaine use } \\
\text { disorder, } \\
\text { cannabis use } \\
\text { disorder, OUD, } \\
\text { amphetamine } \\
\text { use disorder, } \\
\text { sedative/hypnoti } \\
\text { c use disorder }\end{array}$ & $\begin{array}{l}\text { Suicide Mortality (2006- } \\
\text { 2011) }\end{array}$ & $\begin{array}{l}\text { - } 9,087 \text { individuals died by } \\
\text { suicide (34.7 per } 100,00) \text {. } \\
\text { - For men, OUD was the third } \\
\text { largest risk for suicide } \\
\text { - For women, OUD was the } \\
\text { second largest risk for suicide } \\
\text { - Adjusting for mental health } \\
\text { factors, the only substance } \\
\text { specific diagnoses to remain } \\
\text { statistically significant were } \\
\text { alcohol and OUD for women; } \\
\text { for men, any SUD, and all } \\
\text { specific SUDs, remained } \\
\text { significant risk factors for } \\
\text { suicide. } \\
\text { - Post-hoc analyses found a 2-3x } \\
\text { greater suicide rate for women } \\
\text { than men; especially with } \\
\text { respect to alcohol use disorder, } \\
\text { cocaine use disorder, OUD, or a } \\
\text { general SUD diagnosis. }\end{array}$ \\
\hline $\begin{array}{l}\text { Borges et al. } \\
(2000) / \text { USA }\end{array}$ & $\begin{array}{l}\text { Cross Sectional } \\
\text { Study }\end{array}$ & 8,098 (NR) & $\begin{array}{l}1990-1992 \\
\text { National } \\
\text { Comorbidity } \\
\text { Survey (NCS), } \\
\text { individuals aged } \\
\text { 15-54. }\end{array}$ & $\begin{array}{l}\text { - CIDI } \\
\text { - DSM-IIIR }\end{array}$ & $\begin{array}{l}\text { Alcohol, } \\
\text { analgesics, } \\
\text { sedartives, } \\
\text { tranquilizers, } \\
\text { stimulants, } \\
\text { marijuana, } \\
\text { cocaine, } \\
\text { inhalants, } \\
\text { hallucinogens, } \\
\text { heroin }\end{array}$ & SA (lifetme prevalence) & $\begin{array}{l}\text { - Heroin users were } 3.5 \text { times } \\
\text { more likely to attempt suicide } \\
\text { than non-users. } \\
\text { - Current heroin users had an OR } \\
\text { of } 6.1 \text { associated with first-time } \\
\text { SA }\end{array}$ \\
\hline $\begin{array}{l}\text { Bradvik et al. } \\
(2007) / \text { Sweden }\end{array}$ & $\begin{array}{l}\text { Cross Sectional } \\
\text { Study }\end{array}$ & $\begin{array}{l}149 \\
(149)\end{array}$ & $\begin{array}{l}\text { Heroin users } \\
\text { recruited from the } \\
\text { needle exchange } \\
\text { program and the } \\
\text { Addiction Cent } \\
\text { fall of } 2003 .\end{array}$ & & $\begin{array}{l}\text { Heroin, alcohol, } \\
\text { benzodiazepines, } \\
\text { cannabis, central } \\
\text { stimulants }\end{array}$ & $\begin{array}{l}\text { Intentional OD, } \\
\text { unintentional OD, SAs }\end{array}$ & $\begin{array}{l}\text { - Likelihood of reporting a SA } \\
\text { increased with the number of } \\
\text { heroin ODs. } \\
\text { - Of the } 51 \text { persons where heroin } \\
\text { was not used as a means of SA, } \\
18(29 \%) \text { made multiple } \\
\text { attempts. } \\
\text { - When accounting for depressed } \\
\text { mood and other substances of } \\
\text { use (alcohol, benzodiazepines, } \\
\text { cannabis, and central } \\
\text { stimulants) neither showed a } \\
\text { statistically significant impact } \\
\text { on SA. }\end{array}$ \\
\hline
\end{tabular}




\begin{tabular}{|c|c|c|c|c|c|c|c|}
\hline $\begin{array}{c}\text { Author } \\
\text { (Pub year)/ } \\
\text { Country }\end{array}$ & Study Design & $\begin{array}{c}\text { Overall Sample } \\
\text { (OUD) }\end{array}$ & $\begin{array}{c}\text { Sample } \\
\text { Characteristics }\end{array}$ & $\begin{array}{c}\text { Data Sources \& } \\
\text { Measure(s) }\end{array}$ & $\begin{array}{l}\text { Substances } \\
\text { Examined }\end{array}$ & $\begin{array}{c}\text { Outcomes } \\
\text { (Time frame) }\end{array}$ & Key findings/ Summary \\
\hline $\begin{array}{l}\text { Cheek et al. } \\
(2016) / \text { USA }\end{array}$ & $\begin{array}{c}\text { Cross Sectional } \\
\text { Study }\end{array}$ & $10,203(\mathrm{NR})$ & $\begin{array}{l}\text { Adults } 18 \text { yrs }+ \\
\text { with lifetime } \\
\text { history of major } \\
\text { depression and } \\
\text { IDU. }\end{array}$ & $\begin{array}{l}\text { - } 2008-2013 \\
\text { National Survey on } \\
\text { Drug Use and } \\
\text { Health (NSDUH) } \\
\text { - DSM-IV }\end{array}$ & $\begin{array}{l}\text { Heroin, cocaine, } \\
\text { stimulants }\end{array}$ & $\begin{array}{l}\text { Substance use (past 12- } \\
\text { months); SI, suicide } \\
\text { plan(s), SA(s) (2008- } \\
\text { 2013; } 6 \text { years) }\end{array}$ & $\begin{array}{l}\text { - IDUs are more likely to have } \\
\text { increased depressive symptoms } \\
\text { SI, plan, and attempt(s). } \\
\text { - Compared non-heroin IDUs, } \\
\text { those who inject heroin are less } \\
\text { likely to attempt suicide (OR: } \\
\text { 0.92); however, they are more } \\
\text { likely to have SI and develop a } \\
\text { suicide plan (not statistically } \\
\text { significant). }\end{array}$ \\
\hline $\begin{array}{l}\text { Chen et al. } \\
\text { (2009)/Taiwan }\end{array}$ & $\begin{array}{l}\text { Cross Sectional } \\
\text { Study }\end{array}$ & $488(488)$ & $\begin{array}{l}\text { Heroin-dependent } \\
\text { patients recruited } \\
\text { from a methadone } \\
\text { program enrolled } \\
\text { between Sept. } \\
\text { 2006-Jan.2008. }\end{array}$ & $\begin{array}{l}\text { - Clinical Interview } \\
\text { ("Have you } \\
\text { attempted suicide } \\
\text { in the preceding } \\
\text { one-month? Have } \\
\text { you attempted } \\
\text { suicide in your } \\
\text { lifetime?") }\end{array}$ & Heroin, alcohol & $\begin{array}{l}\text { SA(s) (previous month } \\
\text { and lifetime) }\end{array}$ & $\begin{array}{l}\text { - } 10.9 \% \text { of patients had a recent } \\
\text { SA (past } 30 \text { days) and } 17.8 \% \\
\text { reported a lifetime SA. } \\
\text { - Severity of heroin dependence, } \\
\text { depression score, levels of } \\
\text { family support, and number of } \\
\text { stressful life events, were } \\
\text { associated with recent SA(s). } \\
\text { - Multivariable analyses found } \\
\text { that severity of heroin } \\
\text { dependence and needle sharing } \\
\text { were significantly associated } \\
\text { with recent SA. }\end{array}$ \\
\hline $\begin{array}{l}\text { Conner et al. } \\
(2007) / \text { USA }\end{array}$ & Cohort Study & $131(131)$ & $\begin{array}{l}\text { Patients from a } \\
\text { methadone } \\
\text { program at an } \\
\text { urban university } \\
\text { hospital in upstate } \\
\text { New York. }\end{array}$ & & $\begin{array}{l}\text { Methadone, } \\
\text { history of OUD }\end{array}$ & $\begin{array}{l}\text { SA(s) (lifetime and past } \\
\text { year), unintentional OD } \\
\text { (lifetime and past year) }\end{array}$ & $\begin{array}{l}\text { - } 37.4 \%(\mathrm{n}=49) \text { of patients } \\
\text { reported lifetime history of } \\
\text { SA(s). } \\
\text { - Of those with a lifetime history } \\
\text { of SA, } 33(67.4 \%) \text { had } 2+ \\
\text { attempts and } 7(14.3 \%) \text { made } \\
\text { an attempt in the last year. } \\
\text { - Most common methods of SA } \\
\text { were intentional OD }(29, \\
59.2 \%), \text { cutting }(9,18.4 \%) \text {, and } \\
\text { hanging }(5,10.2 \%) . \\
\text { - Multivariable analysis found } \\
\text { that higher belonging score (i.e } \\
\text { greater perceived belonging) } \\
\text { was associated with decreased } \\
\text { probability of history of SA. }\end{array}$ \\
\hline
\end{tabular}




\begin{tabular}{|c|c|c|c|c|c|c|c|}
\hline $\begin{array}{c}\text { Author } \\
\text { (Pub year)/ } \\
\text { Country }\end{array}$ & Study Design & $\begin{array}{c}\text { Overall Sample } \\
\text { (OUD) }\end{array}$ & $\begin{array}{c}\text { Sample } \\
\text { Characteristics }\end{array}$ & $\begin{array}{c}\text { Data Sources \& } \\
\text { Measure(s) }\end{array}$ & $\begin{array}{l}\text { Substances } \\
\text { Examined }\end{array}$ & $\begin{array}{c}\text { Outcomes } \\
\text { (Time frame) }\end{array}$ & Key findings/ Summary \\
\hline $\begin{array}{l}\text { Darke \& Ross } \\
\text { (2001)/Australia }\end{array}$ & $\begin{array}{c}\text { Cross Sectional } \\
\text { Study }\end{array}$ & $223(223)$ & $\begin{array}{l}\text { Methadone } \\
\text { patients recruited } \\
\text { from February- } \\
\text { July } 2000 .\end{array}$ & & $\begin{array}{l}\text { Heroin, polydrug } \\
\text { use }\end{array}$ & $\begin{array}{l}\text { Non-fatal heroin OD, } \\
\text { SA(s) (lifetime) }\end{array}$ & $\begin{array}{l}\text { - } 40 \% \text { of participants reported at } \\
\text { least one SA in their lifetime. } \\
\text { - Males were significantly more } \\
\text { likely to be using heroin at the } \\
\text { time of their most recent SA } \\
\text { compared to females. } \\
\text { - } 8 \% \text { of participants were } \\
\text { receiving psychiatric or } \\
\text { psychological treatment at the } \\
\text { time of their most recent SA. } \\
\text { - } 31 \% \text { of participants had a } \\
\text { history of both an OD } \\
\text { (intentional or unintentional) } \\
\text { and SA(s). } \\
\text { - Multiple logistic regression } \\
\text { analysis found that being } \\
\text { female, parental absence during } \\
\text { childhood, earlier age of first } \\
\text { intoxication, and higher levels } \\
\text { of polydrug use were associated } \\
\text { with history of SA. }\end{array}$ \\
\hline $\begin{array}{l}\text { Darke et al. } \\
\text { (2004)/Australia }\end{array}$ & $\begin{array}{c}\text { Cross Sectional } \\
\text { Study }\end{array}$ & $1,230(1,230)$ & $\begin{array}{l}\text { Current heroin } \\
\text { users and patients } \\
\text { recruited from } \\
\text { different treatment } \\
\text { modalities. }\end{array}$ & $\begin{array}{l}\text { DSM-IV (heroin } \\
\text { dependence) }\end{array}$ & $\begin{array}{l}\text { Heroin, alcohol, } \\
\text { benzodiazepines, } \\
\text { total number of } \\
\text { drug classes used }\end{array}$ & $\begin{array}{l}\text { Lifetime and recent } \\
\text { attempted suicide }\end{array}$ & $\begin{array}{l}\text { - } 34 \% \text { of patients had a history of } \\
\text { SA; females are significantly } \\
\text { more likely than males to report } \\
\text { an attempt (44\% vs } 28 \% \text { ). } \\
\text { - Recurrent SI was reported by } \\
23 \% \text { of patients and } 15 \% \\
\text { reported having a current plan. } \\
\text { - Residential rehabilitation } \\
\text { patients had a significantly } \\
\text { higher level of recurrent SI } \\
\text { compared to other groups. } \\
\text { - Recent SA was associated with } \\
\text { lifetime and recent polydrug } \\
\text { use, recent heroin OD, and } \\
\text { benzodiazepine use. } \\
\text { - Recent history of SA was also } \\
\text { associated with current major } \\
\text { depression, current SI, } \\
\text { diagnosis of borderline } \\
\text { personality disorder, and } \\
\text { diagnosis of PTSD. }\end{array}$ \\
\hline Author & Study Design & $\begin{array}{c}\text { Overall Sample } \\
\text { (OUD) }\end{array}$ & $\begin{array}{c}\text { Sample } \\
\text { Characteristics }\end{array}$ & $\begin{array}{c}\text { Data Sources \& } \\
\text { Measure(s) }\end{array}$ & $\begin{array}{l}\text { Substances } \\
\text { Examined }\end{array}$ & $\begin{array}{c}\text { Outcomes } \\
\text { (Time frame) }\end{array}$ & Key findings/ Summary \\
\hline
\end{tabular}




\begin{tabular}{|c|c|c|c|c|c|c|c|}
\hline $\begin{array}{c}\text { (Pub year)/ } \\
\text { Country }\end{array}$ & & & & & & & \\
\hline $\begin{array}{l}\text { Darke et al. } \\
\text { (2010)/Australia }\end{array}$ & $\begin{array}{l}\text { Cross Sectional } \\
\text { Study }\end{array}$ & $400(300)$ & $\begin{array}{l}\text { Treatment and } \\
\text { non-treatment } \\
\text { seeking drug } \\
\text { users. }\end{array}$ & & $\begin{array}{l}\text { Alcohol, opioids, } \\
\text { psychostimulants } \\
\text {, cannabis }\end{array}$ & $\begin{array}{l}\text { SA(s), SIB, (lifetime, } \\
\text { past 12-months) }\end{array}$ & $\begin{array}{l}\text { - One third of the sample } \\
\text { reported a lifetime history of } \\
\text { SIB, } 20 \% \text { had engaged in SIB } \\
\text { on more than one occasion, and } \\
10 \% \text { had SIB in the past 12- } \\
\text { months. } \\
\text { - Individuals with alcohol and } \\
\text { opioid use were significantly } \\
\text { more likely to have a history of } \\
\text { SIB. } \\
\text { - Females, PTSD diagnosis, } \\
\text { younger age of first } \\
\text { intoxication, and history of SA } \\
\text { were significantly associated } \\
\text { with SIB. } \\
\text { - About } 33 \% \text { of the sample } \\
\text { reported a history of SA, } 17 \% \\
\text { reported multiple SAs, and } 15 \% \\
\text { made an attempt in the past } 12- \\
\text { months. } \\
\text { - } 23 \% \text { of opioid users reported a } \\
\text { history of SA. }\end{array}$ \\
\hline $\begin{array}{l}\text { Darke et al. } \\
\text { (2012)/Australia }\end{array}$ & $\begin{array}{c}\text { Cross Sectional } \\
\text { Study }\end{array}$ & $191(50)$ & $\begin{array}{l}\text { Patients receiving } \\
\text { treatment services } \\
\text { between January } \\
\text { 2009-Januray } \\
2010 \text {. }\end{array}$ & $\begin{array}{l}\text { - Opiate Treatment } \\
\text { Index }\end{array}$ & $\begin{array}{l}\text { Alcohol, opioids, } \\
\text { psychostimulants } \\
\text {, cannabis }\end{array}$ & $\begin{array}{l}\text { SA(s), SIB, (lifetime, } \\
\text { past 12-months) }\end{array}$ & $\begin{array}{l}\text { - Females were more likely than } \\
\text { males to have engaged in SIB } \\
\text { in the preceding year, attempted } \\
\text { suicide, to have multiple } \\
\text { attempts, have an attempt in the } \\
\text { 12-months, and be younger at } \\
\text { their first SA. } \\
\text { - } 43 \% \text { of patients had either } \\
\text { engaged in SIB and/or } \\
\text { attempted suicide, } 18 \% \\
\text { reported both. } \\
\text { - Individuals who experienced } \\
\text { physical assault before age } 16 \\
\text { were significantly more likely } \\
\text { to have engaged in SIB and } \\
\text { have a history of SA compared } \\
\text { to those who experienced an } \\
\text { assault after age sixteen. } \\
\text { - There were no differences in } \\
\text { weekly psychostimulant users, } \\
\text { weekly heroin users, or } \\
\text { combination users in either } \\
\text { lifetime or } 12 \text {-month histories } \\
\text { of self-harm. }\end{array}$ \\
\hline
\end{tabular}




\begin{tabular}{|c|c|c|c|c|c|c|c|}
\hline $\begin{array}{c}\text { Author } \\
\text { (Pub year)/ } \\
\text { Country }\end{array}$ & Study Design & $\begin{array}{c}\text { Overall Sample } \\
\text { (OUD) }\end{array}$ & $\begin{array}{c}\text { Sample } \\
\text { Characteristics }\end{array}$ & $\begin{array}{c}\text { Data Sources \& } \\
\text { Measure(s) }\end{array}$ & $\begin{array}{l}\text { Substances } \\
\text { Examined }\end{array}$ & $\begin{array}{c}\text { Outcomes } \\
\text { (Time frame) }\end{array}$ & Key findings/ Summary \\
\hline $\begin{array}{l}\text { Darke et al. } \\
\text { (2015)/Australia }\end{array}$ & Cohort Study & $615(413)$ & $\begin{array}{l}\text { Australian } \\
\text { Treatment } \\
\text { Outcomes Study } \\
\text { (ATOS), 11-year } \\
\text { follow-up study. }\end{array}$ & $\begin{array}{l}\text { - Composite } \\
\text { International } \\
\text { Diagnostic } \\
\text { Interview } 2.1 \\
\text { (CIDI) } \\
\text { - Opiate Treatment } \\
\text { Index }\end{array}$ & $\begin{array}{l}\text { Heroin, nicotine, } \\
\text { alcohol, } \\
\text { cannabis, } \\
\text { benzodiazepines, } \\
\text { prescription } \\
\text { opioids }\end{array}$ & $\begin{array}{l}\text { SA(s) (lifetime, 12- } \\
\text { months, 24-months, 36- } \\
\text { months), current SI, } \\
\text { suicidal plan(s) }\end{array}$ & $\begin{array}{l}\text { - } 34.7 \% \text { of participants used } \\
\text { heroin in the } 12 \text {-months prior to } \\
\text { follow-up, } 24.8 \% \text { used in the } \\
\text { month prior, and } 9.7 \% \text { reported } \\
\text { daily heroin use. } \\
\text { - } 42.2 \% \text { of ATOS participants } \\
\text { reported ever having made a } \\
\text { SA and } 18.6 \% \text { reported making } \\
\text { multiple attempts at } 11 \text {-year } \\
\text { follow-up. } \\
\text { - Participants that reported a } \\
\text { recent SA (12 months prior to } \\
\text { follow-up), having a current } \\
\text { plan, and/or SI were more } \\
\text { likely to live alone, be } \\
\text { unemployed, meet criteria for } \\
\text { Major Depression and PTSD, } \\
\text { be in poorer health, and report } \\
\text { more extensive polydrug use. } \\
\text { - Diagnosis of Major Depression } \\
\text { increased the odds of SA by } \\
1.68 \text { times, each extra drug } \\
\text { class used increased odds by } \\
1.39 \text { times }\end{array}$ \\
\hline $\begin{array}{l}\text { de los Cobos et al. } \\
(2007) / \text { Spain }\end{array}$ & $\begin{array}{c}\text { Cross Sectional } \\
\text { Study }\end{array}$ & $\begin{array}{l}164 \\
(164)\end{array}$ & $\begin{array}{l}\text { Opioid-abstinent } \\
\text { or hospitalized } \\
\text { patients with } \\
\text { OUD. }\end{array}$ & $\begin{array}{l}\text { - Clinical evaluation } \\
\text { conducted by } \\
\text { psychiatrist }\end{array}$ & Heroin & $\begin{array}{l}\text { SIBs and ODs (during } \\
\text { course of patients } \\
\text { reported heroin } \\
\text { dependence); history of } \\
\text { bulimia, SA(s) (lifetime) }\end{array}$ & $\begin{array}{l}\text { - } 87.8 \% \text { of the heroin-dependent } \\
\text { patients interviewed had a } \\
\text { history of SIB. } \\
\text { - Most common SIB: hitting } \\
\text { extremities ( } 77.4 \%) \text {, picking } \\
\text { scabs }(52.4 \%) \text {, head-butting } \\
(43.3 \%) \text {, cutting }(25 \%) \text {, biting } \\
(20.7 \%) \text {, carving signs }(15.2 \%) \text {, } \\
\text { scratching (14.6\%), burns } \\
(14 \%) \text { and pulling out hair } \\
(9.1 \%) \text {. } \\
25.6 \% \text { had a history of SA(s), } \\
59.1 \% \text { had an accidental OD(s) }\end{array}$ \\
\hline
\end{tabular}




\begin{tabular}{|c|c|c|c|c|c|c|c|}
\hline $\begin{array}{c}\text { Author } \\
\text { (Pub year)/ } \\
\text { Country }\end{array}$ & Study Design & $\begin{array}{c}\text { Overall Sample } \\
\text { (OUD) }\end{array}$ & $\begin{array}{c}\text { Sample } \\
\text { Characteristics }\end{array}$ & $\begin{array}{c}\text { Data Sources \& } \\
\text { Measure(s) }\end{array}$ & $\begin{array}{c}\text { Substances } \\
\text { Examined }\end{array}$ & $\begin{array}{l}\text { Outcomes } \\
\text { (Time frame) }\end{array}$ & Key findings/ Summary \\
\hline $\begin{array}{l}\text { Dragisic et al. } \\
\text { (2015)/Bosnia and } \\
\text { Herzegovina }\end{array}$ & $\begin{array}{l}\text { Case-Control } \\
\text { Study }\end{array}$ & $200(200)$ & $\begin{array}{l}\text { Individuals } \\
\text { recovering from } \\
\text { OUD with and } \\
\text { without history of } \\
\text { SA. }\end{array}$ & - ICD-10 & Opioids & SA(s) (lifetime) & $\begin{array}{l}\text { - Individuals with OUD who } \\
\text { attempted suicide were more } \\
\text { likely to be unmarried or } \\
\text { divorced compared to those } \\
\text { with OUD who have not } \\
\text { attempted suicide. } \\
\text { - Clients with history of SA more } \\
\text { commonly had psychotic } \\
\text { disorders, drug addiction, and } \\
\text { family history of SAs. } \\
\text { Persons with OUD and } \\
\text { attempted suicide also have a } \\
\text { significantly longer history of } \\
\text { addiction duration (i.e. } \\
\text { substance use) and IDU. }\end{array}$ \\
\hline $\begin{array}{l}\text { Ghaffari-Nejad et } \\
\text { al. (2011)/Iran }\end{array}$ & $\begin{array}{l}\text { Case-Control } \\
\text { Study }\end{array}$ & $70(70)$ & $\begin{array}{l}\text { Hospitalized } \\
\text { patients for SUD. }\end{array}$ & $\begin{array}{l}\text { - DSM-V } \\
\text { - California Suicide } \\
\text { Risk Assessment } \\
\text { Questionnaire }\end{array}$ & Opioids & Suicide Risk & $\begin{array}{l}\text { - Cases were patients with OUD; } \\
\text { controls were peers without } \\
\text { OUD } \\
\text { - Suicide risk wasn't statistically } \\
\text { significant between the case } \\
\text { and control group. } \\
\text { - Individuals with OUD had a } \\
\text { significantly higher depression } \\
\text { scores than controls }\end{array}$ \\
\hline $\begin{array}{l}\text { Gilmore et al. } \\
(2018) / \text { USA }\end{array}$ & $\begin{array}{c}\text { Cross Sectional } \\
\text { Study }\end{array}$ & $60(24)$ & $\begin{array}{l}\text { Patients who } \\
\text { received sexual } \\
\text { assault treatment } \\
\text { in the ED. }\end{array}$ & $\begin{array}{l}\text { - Self-report } \\
\text { - Patient Health } \\
\text { Questionnaire-9 } \\
\text { (SI) }\end{array}$ & Opioids & SI (past 2 weeks) & $\begin{array}{l}\text { - Linear regression analysis } \\
\text { found a significant association } \\
\text { between prescription opioid use } \\
\text { and SI. } \\
\text { - Those with a prior sexual } \\
\text { assault and OUD reported more } \\
\text { severe SI }\end{array}$ \\
\hline $\begin{array}{l}\text { Hakansson et al. } \\
\text { (2010)/Sweden }\end{array}$ & $\begin{array}{c}\text { Cross Sectional } \\
\text { Study }\end{array}$ & $6,836(2,346)$ & $\begin{array}{l}\text { Entrants in the } \\
\text { Swedish criminal } \\
\text { justice system } \\
\text { databased between } \\
2001 \text { and August } \\
2006 \text {. }\end{array}$ & $\begin{array}{l}\text { - Addiction Severity } \\
\text { Index }\end{array}$ & $\begin{array}{l}\text { Alcohol, heroin, } \\
\text { methadone, } \\
\text { opioids, } \\
\text { tranquilizers, } \\
\text { amphetamines, } \\
\text { cocaine, } \\
\text { cannabis, } \\
\text { solvents, } \\
\text { hallucinogens }\end{array}$ & SA(s) (lifetime) & $\begin{array}{l}\text { - } 21 \%(\mathrm{n}=1,453) \text { of persons } \\
\text { reported a SA. } \\
\text { - Logistic regression found: older } \\
\text { age, female gender, history of } \\
\text { binge drinking, IDU, delirium } \\
\text { tremens, OD, chronic medical } \\
\text { problems, maternal alcohol or } \\
\text { psychiatric problems, paternal } \\
\text { alcohol problems, history of } \\
\text { emotional, physical, and sexual } \\
\text { abuse, history of depression, } \\
\text { anxiety, hallucinations, eating } \\
\text { disorders, and difficulty } \\
\text { controlling violent behavior }\end{array}$ \\
\hline
\end{tabular}




\begin{tabular}{|c|c|c|c|c|c|c|c|}
\hline & & & & & & & $\begin{array}{l}\text { were independently associated } \\
\text { with history of SA. }\end{array}$ \\
\hline $\begin{array}{c}\text { Author } \\
\text { (Pub year)/ } \\
\text { Country }\end{array}$ & Study Design & $\begin{array}{c}\text { Overall Sample } \\
\text { (OUD) }\end{array}$ & $\begin{array}{c}\text { Sample } \\
\text { Characteristics }\end{array}$ & $\begin{array}{l}\text { Data Sources \& } \\
\text { Measure(s) }\end{array}$ & $\begin{array}{l}\text { Substances } \\
\text { Examined }\end{array}$ & $\begin{array}{c}\text { Outcomes } \\
\text { (Time frame) }\end{array}$ & Key findings/ Summary \\
\hline $\begin{array}{l}\text { Harned et al. } \\
(2006) / \text { USA }\end{array}$ & $\begin{array}{l}\text { Cross Sectional } \\
\text { Study }\end{array}$ & $65(9)$ & $\begin{array}{l}\text { A sample of } \\
\text { outpatient women } \\
\text { with PTSD and } \\
\text { SUD. }\end{array}$ & $\begin{array}{l}\text { DSM-IV } \\
\text { - Traumatic History } \\
\text { Questionnaire } \\
\text { - Suicidal Behaviors } \\
\text { Questionnaire, 13- } \\
\text { item revised } \\
\text { version }\end{array}$ & $\begin{array}{l}\text { Alcohol, } \\
\text { cocaine, } \\
\text { cannabis, } \\
\text { opioids, } \\
\text { sedative- } \\
\text { hypnotics, } \\
\text { amphetamines, } \\
\text { hallucinogens }\end{array}$ & $\begin{array}{l}\text { Self-harm ideation, self- } \\
\text { harm, SI, SA(s) (past } \\
\text { three months) }\end{array}$ & $\begin{array}{l}\text { - } 13.8 \% \text { engaged in SIB, } 10.8 \% \\
\text { attempted suicide, and } 7.7 \% \\
\text { had both SIB and attempted } \\
\text { suicide ( } \mathrm{n}=21 \text { total). } \\
\text { - Of these } 21 \text { women, } 61.9 \% \\
\text { reported drinking alcohol or } \\
\text { using drugs immediately before } \\
\text { or during the SA/self-harm } \\
\text { episode. } \\
\text { - Recent history of SIB and/or } \\
\text { attempted suicide were more } \\
\text { likely to have diagnoses of } \\
\text { AUD and polysubstance } \\
\text { dependence and less likely to } \\
\text { meet criteria OUD. }\end{array}$ \\
\hline $\begin{array}{l}\text { Havens et al. } \\
(2004) / \text { USA }\end{array}$ & $\begin{array}{l}\text { Cross Sectional } \\
\text { Study }\end{array}$ & 2,219 (NR) & $\begin{array}{l}\text { IDUs } 15-30 \text { yrs } \\
\text { old recruited from } \\
1997 \text { to } 1999 .\end{array}$ & & $\begin{array}{l}\text { Heroin, cocaine, } \\
\text { marijuana, } \\
\text { alcohol }\end{array}$ & $\begin{array}{l}\text { SI, SA(s), and suicide } \\
\text { plan(s)(6-months) }\end{array}$ & $\begin{array}{l}\text { - } 68.9 \% \text { of participants were } \\
\text { using heroin daily. } \\
\text { - In the past 6-months, receiving } \\
\text { money for sex was associated } \\
\text { with recent SA(s) } \\
\text { - Daily heroin, speedball, } \\
\text { marijuana, or IDU was not } \\
\text { associated with recent SA(s) } \\
\text { - Polydrug use was significantly } \\
\text { associate with recent SA }\end{array}$ \\
\hline $\begin{array}{l}\text { Heale et al. } \\
(2003) / \text { Australia }\end{array}$ & $\begin{array}{l}\text { Cross Sectional } \\
\text { Study }\end{array}$ & $256(256)$ & $\begin{array}{l}\text { Heroin OD } \\
\text { survivors } \\
\text { resuscitated by } \\
\text { paramedics } \\
\text { between July } \\
\text { 1999-May } 2001 \text {. }\end{array}$ & & $\begin{array}{l}\text { Heroin, } \\
\text { benzodiazepines, } \\
\text { alcohol }\end{array}$ & $\begin{array}{l}\text { SA(s), intentional OD } \\
\text { (lifetime, past 6-months) }\end{array}$ & $\begin{array}{l}\text { - } 17 \% \text { reported an intentional OD } \\
(\mathrm{n}=42) ; 67 \% \text { reporting at least } \\
\text { one in the past 6-months. } \\
\text { - Primary reasons for intentional } \\
\text { OD included: relationship } \\
\text { breakdown }(3 / 9) \text {, family } \\
\text { problems }(2 / 9) \text {, and treatment } \\
\text { discharge } \\
\text { - Survivors of recent intentional } \\
\text { ODs reported using a } \\
\text { statistically significantly higher } \\
\text { mean amount of heroin }\end{array}$ \\
\hline
\end{tabular}




\begin{tabular}{|c|c|c|c|c|c|c|c|}
\hline $\begin{array}{c}\text { Author } \\
\text { (Pub year)/ } \\
\text { Country }\end{array}$ & Study Design & $\begin{array}{c}\text { Overall Sample } \\
\text { (OUD) }\end{array}$ & $\begin{array}{c}\text { Sample } \\
\text { Characteristics }\end{array}$ & $\begin{array}{c}\text { Data Sources \& } \\
\text { Measure(s) }\end{array}$ & $\begin{array}{l}\text { Substances } \\
\text { Examined }\end{array}$ & $\begin{array}{c}\text { Outcomes } \\
\text { (Time frame) }\end{array}$ & Key findings/ Summary \\
\hline $\begin{array}{l}\text { Icick et al. } \\
\text { (2017)/France }\end{array}$ & $\begin{array}{c}\text { Cross Sectional } \\
\text { Study }\end{array}$ & $433(217)$ & $\begin{array}{l}\text { Treatment seeking } \\
\text { outpatients } \\
\text { recruited between } \\
\text { April } 2008 \text { \& } \\
\text { April } 2016 .\end{array}$ & $\begin{array}{l}\text { DSM-IV-TR } \\
\text { - Diagnostic } \\
\text { Interview for } \\
\text { Genetic Studies } \\
\text { v4.0, "suicide } \\
\text { section" (DIGS } \\
4.0)\end{array}$ & $\begin{array}{l}\text { Opioids, } \\
\text { nicotine, } \\
\text { cocaine, alcohol, } \\
\text { sedatives, } \\
\text { cannabis, } \\
\text { multiple SUDs }\end{array}$ & SA(s) (lifetiome) & $\begin{array}{l}\text { - Patients reporting serious and } \\
\text { non-serious SAs had earlier } \\
\text { onset of OUD and tobacco } \\
\text { smoking. } \\
\text { - Women were less likely to have } \\
\text { a serious SA when they } \\
\text { reported any history of MOUD. } \\
\text { - Women with current mood } \\
\text { disorder and OUD were less } \\
\text { likely to present with a serious } \\
\text { SA. }\end{array}$ \\
\hline $\begin{array}{l}\text { Ilgen et al. } \\
(2009) / \text { USA }\end{array}$ & $\begin{array}{l}\text { Cross Sectional } \\
\text { Study }\end{array}$ & 488 (488) & $\begin{array}{l}\text { Individuals, 18-63 } \\
\text { yrs old receiving } \\
\text { SUD treatment. }\end{array}$ & $\begin{array}{l}\text { - National Death } \\
\text { Index (NDI) } \\
\text { - ICD-10 } \\
\text { - ICD-9-CM }\end{array}$ & $\begin{array}{l}\text { Alcohol, } \\
\text { cocaine, } \\
\text { cannabis, } \\
\text { opioids, } \\
\text { amphetamine, } \\
\text { barbiturate, } \\
\text { multiple } \\
\text { substances }\end{array}$ & $\begin{array}{l}\text { Violent suicide, } \\
\text { nonviolent suicide (FY } \\
\text { 2002-2006) }\end{array}$ & $\begin{array}{l}\text { - OUD and polysubstance use } \\
\text { were associated with greater } \\
\text { likelihood of nonviolent } \\
\text { suicide. } \\
\text { - Logistic regression analyses } \\
\text { found that among those who } \\
\text { died by suicide, cocaine, OUD, } \\
\text { and multiple SUDs were all } \\
\text { associated with greater risk of } \\
\text { nonviolent compared to violent } \\
\text { suicide. } \\
\text { - Patients with OUD who died by } \\
\text { suicide have an odds ratio of } \\
0.76 \text { of using violent means } \\
\text { compared to an odds ratio of } \\
1.67 \text { for nonviolent means. }\end{array}$ \\
\hline
\end{tabular}




\begin{tabular}{|c|c|c|c|c|c|c|c|}
\hline $\begin{array}{c}\text { Author } \\
\text { (Pub year)/ } \\
\text { Country }\end{array}$ & Study Design & $\begin{array}{c}\text { Overall Sample } \\
\text { (OUD) }\end{array}$ & $\begin{array}{c}\text { Sample } \\
\text { Characteristics }\end{array}$ & $\begin{array}{c}\text { Data Sources \& } \\
\text { Measure(s) }\end{array}$ & $\begin{array}{l}\text { Substances } \\
\text { Examined }\end{array}$ & $\begin{array}{c}\text { Outcomes } \\
\text { (Time frame) }\end{array}$ & Key findings/ Summary \\
\hline $\begin{array}{l}\text { Jin et al. } \\
(2013) / \text { China }\end{array}$ & $\begin{array}{l}\text { Case-Control } \\
\text { Study }\end{array}$ & $607(406)$ & $\begin{array}{l}\mathrm{HIV}+(\mathrm{n}=204) \text { and } \\
\mathrm{HIV}-(\mathrm{n}=202) \\
\text { patients with } \\
\text { history of heroin } \\
\text { IDU in methadone } \\
\text { treatment. }\end{array}$ & $\begin{array}{l}\text { - Chinese version of } \\
\text { the Composite } \\
\text { International } \\
\text { Diagnostic } \\
\text { Interview (CIDI } \\
\text { v3.0) } \\
\text { - Item } 9 \text { of the Beck } \\
\text { Depression } \\
\text { Inventory-II }\end{array}$ & Heroin, alcohol & SI (past 2 weeks) & $\begin{array}{l}\text { - Factors significantly associated } \\
\text { with current SI included being } \\
\text { unmarried or unemployed, } \\
\text { having a lifetime (past) history } \\
\text { of major depression, or lifetime } \\
\text { alcohol use disorder or heroin } \\
\text { use disorder. } \\
\text { - Among treated IDUs ( } \mathrm{n}=407 \text { ), } \\
\text { past history of major } \\
\text { depression, or past AUD, and } \\
\text { higher BDI-II total score were } \\
\text { associated with current } \\
\text { suicidality } \\
\text { - Longer duration of IDU and } \\
\text { poor social support were also } \\
\text { significantly associated with } \\
\text { current suicidality } \\
\text { - Multivariable predictors of } \\
\text { suicidality were HIV-related } \\
\text { stress, social support, and prior } \\
\text { history of major depression. } \\
\text { - Suicidality among HIV- treated } \\
\text { IDUs, was best predicted by } \\
\text { prior history of major } \\
\text { depressive disorder and prior } \\
\text { history of AUD. } \\
\text { - Prior major depression } \\
\text { increased risk by 6-fold and } \\
\text { past AUD increased risk by 3- } \\
\text { fold. }\end{array}$ \\
\hline $\begin{array}{l}\text { Kalyoncu et al. } \\
\text { (2007)/Turkey }\end{array}$ & $\begin{array}{l}\text { Cross Sectional } \\
\text { Study }\end{array}$ & $108(108)$ & $\begin{array}{l}\text { Patients } 18-24 \text { yrs } \\
\text { old receiving } \\
\text { inpatient treatment } \\
\text { for OUD. }\end{array}$ & - DSM-IV & $\begin{array}{l}\text { Alcohol, heroin, } \\
\text { overall drug use }\end{array}$ & SA(s) (past month) & $\begin{array}{l}\text { - } 37.0 \%(\mathrm{n}=40) \text { had a history of } \\
\text { SA. } \\
\text { - Childhood trauma } \\
\text { questionnaire scores were } \\
\text { significantly higher among } \\
\text { patients with a history of SA } \\
\text { than patients without a history } \\
\text { of SA. } \\
\text { - Patients with a SA also had a } \\
\text { greater likelihood of meeting } \\
\text { DSM-IV criteria for another } \\
\text { current psychiatric disorder, } \\
\text { specifically antisocial } \\
\text { personality disorder. }\end{array}$ \\
\hline
\end{tabular}




\begin{tabular}{|c|c|c|c|c|c|c|c|}
\hline $\begin{array}{c}\text { Author } \\
\text { (Pub year)/ } \\
\text { Country }\end{array}$ & Study Design & $\begin{array}{l}\text { Overall Sample } \\
\text { (OUD) }\end{array}$ & $\begin{array}{c}\text { Sample } \\
\text { Characteristics }\end{array}$ & $\begin{array}{c}\text { Data Sources \& } \\
\text { Measure(s) }\end{array}$ & $\begin{array}{l}\text { Substances } \\
\text { Examined }\end{array}$ & $\begin{array}{c}\text { Outcomes } \\
\text { (Time frame) }\end{array}$ & Key findings/ Summary \\
\hline $\begin{array}{l}\text { Kazour et al. } \\
(2016) / \text { Lebanon }\end{array}$ & $\begin{array}{l}\text { Case-Control } \\
\text { Study }\end{array}$ & $122(61)$ & $\begin{array}{l}\text { Patients with } \\
\text { OUD. }\end{array}$ & $\begin{array}{ll}- & \text { DSM-IV } \\
- & \text { Beck SI Scale } \\
& (\text { BSI })\end{array}$ & $\begin{array}{l}\text { Opioids, } \\
\text { cocaine, } \\
\text { cannabis, ecstasy } \\
\text { (MDMA), LSD, } \\
\text { amphetamines, } \\
\text { benzodiazepines, } \\
\text { alcohol }\end{array}$ & $\begin{array}{l}\text { SA(s) (lifetime), SI } \\
\text { (current) }\end{array}$ & $\begin{array}{l}\text { - } 37.7 \%(\mathrm{n}=23) \text { of the heroin } \\
\text { dependent group had a personal } \\
\text { history of SA; the most } \\
\text { frequent method was } \\
\text { intentional heroin OD, oral } \\
\text { medication OD and cutting. } \\
\text { - History of SA was associated } \\
\text { with females, had younger age } \\
\text { of first substance use, and } \\
\text { OUD. } \\
\text { - Number of SAs was } \\
\text { significantly associated with } \\
\text { number of previous detox } \\
\text { admissions and number SAs } \\
\text { among family. } \\
\text { - Subjects with OUD, } 9 \text { had } \\
\text { current SI. } \\
\text { - Patients with current SI did not } \\
\text { have higher rates of OD, but } \\
\text { they did have significantly } \\
\text { higher rates of intentional OD. }\end{array}$ \\
\hline $\begin{array}{l}\text { Kuramoto et al. } \\
(2012) / \text { USA }\end{array}$ & $\begin{array}{l}\text { Cross Sectional } \\
\text { Study }\end{array}$ & $37,933(7,468)$ & $\begin{array}{l}2009 \text { National } \\
\text { Survey on Drug } \\
\text { Use and Health } \\
\text { (NSDUH). }\end{array}$ & - DSM-IV & $\begin{array}{l}\text { Non-medical } \\
\text { prescription } \\
\text { opioids }\end{array}$ & $\begin{array}{l}\text { SI, SA(s) (past 12- } \\
\text { months) }\end{array}$ & $\begin{array}{l}\text { - Approximately } 7 \% \text { of the } \\
\text { former nonmedical prescription } \\
\text { opioid users ( } \mathrm{n}=310), 11 \% \text { of } \\
\text { the persistent users }(\mathrm{n}=287) \text {, } \\
\text { and } 9 \% \text { of the recent-onset } \\
\text { users ( }=178 \text { ) reported SI } \\
\text { compared to the } 3 \% \text { of never } \\
\text { users who reported ideation. } \\
\text { - A greater proportion of } \\
\text { persistent (17\%) and recent- } \\
\text { onset ( } 19 \%) \text { users with ideation } \\
\text { also reported SA as compared } \\
\text { with former and never users. } \\
\text { 23\% of nonmedical users with } \\
\text { past-year prescription opioid } \\
\text { disorder reported SI } \\
\text { - Individuals with OUD had } \\
\text { more than threefold increased } \\
\text { odds for reporting SI. }\end{array}$ \\
\hline
\end{tabular}




\begin{tabular}{|c|c|c|c|c|c|c|c|}
\hline $\begin{array}{c}\text { Author } \\
\text { (Pub year)/ } \\
\text { Country }\end{array}$ & Study Design & $\begin{array}{c}\text { Overall Sample } \\
\text { (OUD) }\end{array}$ & $\begin{array}{c}\text { Sample } \\
\text { Characteristics }\end{array}$ & $\begin{array}{c}\text { Data Sources \& } \\
\text { Measure(s) }\end{array}$ & $\begin{array}{l}\text { Substances } \\
\text { Examined }\end{array}$ & $\begin{array}{c}\text { Outcomes } \\
\text { (Time frame) }\end{array}$ & Key findings/ Summary \\
\hline $\begin{array}{l}\text { Lavania et al. } \\
\text { (2012)/India }\end{array}$ & $\begin{array}{c}\text { Cross Sectional } \\
\text { Study }\end{array}$ & $60(27)$ & $\begin{array}{l}\text { Nondepressed, } \\
\text { male inpatients } \\
18-60 \text { yrs old with } \\
\text { SUD. }\end{array}$ & $\begin{array}{ll}\text { - } & \text { Addiction } \\
\text { Severity Index } \\
\text { (ASI) } \\
\text { - } \text { ICD-10 }\end{array}$ & $\begin{array}{l}\text { Opioids, alcohol, } \\
\text { unspecified } \\
\text { others }\end{array}$ & $\begin{array}{l}\text { Self-injurious behavior } \\
\text { (SIB) (lifetime) }\end{array}$ & $\begin{array}{l}\text { - Higher rates of OUD were } \\
\text { found in people who reported } \\
\text { SIB. } \\
\text { - Individuals with SIB had } \\
\text { significantly higher rates of } \\
\text { OUD, risk of isolation, the } \\
\text { number of life events, anger } \\
\text { trait and anger expression, } \\
\text { personality disorder, number of } \\
\text { substance use problems, and } \\
\text { lower social function. }\end{array}$ \\
\hline $\begin{array}{l}\text { Maloney et al. } \\
\text { (2009)/Australia }\end{array}$ & $\begin{array}{l}\text { Case-Control } \\
\text { Study }\end{array}$ & $1,125(726)$ & $\begin{array}{l}\text { Individuals } \\
\text { receiving MOUD } \\
\text { treatment. }\end{array}$ & • DSM-IV & $\begin{array}{l}\text { Opioids, } \\
\text { cannabis, } \\
\text { sedatives, } \\
\text { stimulants, } \\
\text { cocaine, alcohol, } \\
\text { tobacco }\end{array}$ & $\begin{array}{l}\text { SI (lifetime), persistent } \\
\text { SI (lifetime), suicide plan } \\
\text { (lifetime), SA(s) } \\
\text { (lifetime, past 12- } \\
\text { months) }\end{array}$ & $\begin{array}{l}\text { - Cases were recruited from } \\
\text { opioid maintenance treatment. } \\
\text { - Controls were recruited from } \\
\text { the community in the same } \\
\text { geographic area as the } \\
\text { treatment centers. } \\
\text { - Female cases were significantly } \\
\text { more likely to attempt suicide } \\
\text { prior to the onset of heroin use } \\
\text { compared to male cases ( } 68 \% \\
\text { vs } 46 \% \text { ). } \\
\text { - Cases who reported a lifetime } \\
\text { SA were significantly more } \\
\text { likely to meet DSM-IV criteria } \\
\text { for dependence for each drug } \\
\text { class examined and psychiatric } \\
\text { disorders assessed. } \\
\text { OUD was not a significant risk } \\
\text { factor for SAs over and above } \\
\text { the other identified risk factors. }\end{array}$ \\
\hline
\end{tabular}




\begin{tabular}{|c|c|c|c|c|c|c|c|}
\hline $\begin{array}{c}\text { Author } \\
\text { (Pub year)/ } \\
\text { Country }\end{array}$ & Study Design & $\begin{array}{l}\text { Overall Sample } \\
\text { (OUD) }\end{array}$ & $\begin{array}{c}\text { Sample } \\
\text { Characteristics }\end{array}$ & $\begin{array}{c}\text { Data Sources \& } \\
\text { Measure(s) }\end{array}$ & $\begin{array}{l}\text { Substances } \\
\text { Examined }\end{array}$ & $\begin{array}{l}\text { Outcomes } \\
\text { (Time frame) }\end{array}$ & Key findings/ Summary \\
\hline $\begin{array}{l}\text { Maloney et al. } \\
\text { (2010)/Australia }\end{array}$ & $\begin{array}{l}\text { Case-Control } \\
\text { Study }\end{array}$ & $966(686)$ & $\begin{array}{l}\text { Individuals with } \\
\text { and without OUD. }\end{array}$ & - DSM-IV & $\begin{array}{l}\text { Cannabis, } \\
\text { sedatives, } \\
\text { opioids, } \\
\text { stimulants, } \\
\text { cocaine, } \\
\text { nicotine, alcohol }\end{array}$ & $\begin{array}{l}\text { SA(s), Self-injurious } \\
\text { behavior (SIB), Both SIB } \\
\& \text { SA(s) (lifetime) }\end{array}$ & $\begin{array}{l}\text { - Female cases were significantly } \\
\text { more likely to report a history } \\
\text { of SIB compared to male cases. } \\
\text { - The SIB group were } \\
\text { significantly more likely to be } \\
\text { female, younger, completed } \\
\text { more than } 10 \text { years of } \\
\text { schooling, report every } \\
\text { childhood maltreatment } \\
\text { subtype, meet criteria for } \\
\text { alcohol dependence, and } \\
\text { criteria for dependence on more } \\
\text { lifetime drug classes. } \\
\text { - The SIB and SA were more } \\
\text { likely to report multiple } \\
\text { lifetime SAs compared to those } \\
\text { in the SA only group and had a } \\
\text { significantly increased risk for } \\
\text { screening positively for BPD. }\end{array}$ \\
\hline $\begin{array}{l}\text { Marchand et al. } \\
\text { (2017)/Canada }\end{array}$ & $\begin{array}{c}\text { Cross Sectional } \\
\text { Study }\end{array}$ & $176(176)$ & $\begin{array}{l}\text { Long-term opioid } \\
\text { dependent } \\
\text { individuals } \\
\text { recruited between } \\
\text { December } 2011 \\
\text { and June } 2013 \text {. }\end{array}$ & $\begin{array}{l}\text { - Composite } \\
\text { International } \\
\text { Diagnostic } \\
\text { Interview-Major } \\
\text { Depression } \\
\text { Module }\end{array}$ & $\begin{array}{l}\text { Opioids, illicit } \\
\text { stimulants }\end{array}$ & $\begin{array}{l}\text { SI, suicide plan(s), SA(s) } \\
\text { (lifetime) }\end{array}$ & $\begin{array}{l}\text { - Of those with lifetime } \mathrm{SI}(\mathrm{n}= \\
\text { 77), plans for suicide were } \\
\text { reported by } 62.3 \%(\mathrm{n}=48) \text {, and } \\
75.3 \% \text { (n=58) reported SAs. } \\
\text { - Each unit increase in childhood } \\
\text { emotional neglect score and } \\
\text { number of potentially traumatic } \\
\text { life events, the adjusted odds of } \\
\text { lifetime SI increased by } 9 \% \text { and } \\
13 \% \text {, respectively. } \\
\text { - History of depression, anxiety, } \\
\text { childhood emotional neglect, } \\
\text { and the number of lifetime } \\
\text { traumatic events were } \\
\text { significantly associated with } \\
\text { higher odds of SI. } \\
\text { - For women histories of } \\
\text { depression and anxiety } \\
\text { remained independently } \\
\text { associated with lifetime SI } \\
\text { among women } \\
\text { - For men, childhood emotional } \\
\text { neglect and number of lifetime } \\
\text { traumatic events were } \\
\text { independently associated with } \\
\text { lifetime SI. }\end{array}$ \\
\hline
\end{tabular}




\begin{tabular}{|c|c|c|c|c|c|c|c|}
\hline $\begin{array}{c}\text { Author } \\
\text { (Pub year)/ } \\
\text { Country }\end{array}$ & Study Design & $\begin{array}{c}\text { Overall Sample } \\
\text { (OUD) }\end{array}$ & $\begin{array}{c}\text { Sample } \\
\text { Characteristics }\end{array}$ & $\begin{array}{c}\text { Data Sources \& } \\
\text { Measure(s) }\end{array}$ & $\begin{array}{l}\text { Substances } \\
\text { Examined }\end{array}$ & $\begin{array}{c}\text { Outcomes } \\
\text { (Time frame) }\end{array}$ & Key findings/ Summary \\
\hline $\begin{array}{l}\text { Maremmani et al. } \\
(2007) / \text { Italy }\end{array}$ & $\begin{array}{c}\text { Cross Sectional } \\
\text { Study }\end{array}$ & $616(616)$ & $\begin{array}{l}\text { Individuals with } \\
\text { OUD requesting } \\
\text { outpatient } \\
\text { treatment between } \\
1995 \text { and } 2003 .\end{array}$ & $\begin{array}{l}\text { - } \text { DSM-IV } \\
\text { - Drug Addiction } \\
\text { History Rating } \\
\text { Scale (DAH-RS) } \\
\text { - SCL-90-item 15 } \\
\text { (SI) }\end{array}$ & $\begin{array}{l}\text { Alcohol, opioids, } \\
\text { CNS } \\
\text { depressants, } \\
\text { CNS stimulants, } \\
\text { hallucinogens, } \\
\text { phencyclidine, } \\
\text { cannabis, } \\
\text { inhalants, poly- } \\
\text { substance use }\end{array}$ & SI (past week) & $\begin{array}{l}\text { - Suicidal thoughts during the } \\
\text { past week were reported by } 199 \\
\text { patients ( } 29.1 \%) \text {. } \\
\text { - SI did not correlate with } \\
\text { patterns of heroin use (presence } \\
\text { of periodic self-detoxification } \\
\text { vs. uninterrupted use), } \\
\text { frequency of intake (daily vs. } \\
\text { sporadic), age of first substance } \\
\text { use or age at first treatment. } \\
\text { - A significant association was } \\
\text { present between duration of } \\
\text { addiction and SI. } \\
\text { - Odds of SI were higher for } \\
\text { patients receiving welfare } \\
\text { benefits }(\mathrm{OR}=1.69) \text {, with } \\
\text { bipolar spectrum disorder (OR } \\
=1.42), \text { the unemployed (OR }= \\
1.37) \text {, those with early onset of } \\
\text { OUD (OR = 1.36), those living } \\
\text { alone (OR = 1.33), and those } \\
\text { with problems in organizing. }\end{array}$ \\
\hline $\begin{array}{l}\text { Masferrer et al. } \\
\text { (2016)/Spain }\end{array}$ & $\begin{array}{c}\text { Cross Sectional } \\
\text { Study }\end{array}$ & $196(36)$ & $\begin{array}{l}\text { Bereaved SUD } \\
\text { patients, abstinent } \\
\text { for at least 30- } \\
\text { days. }\end{array}$ & $\begin{array}{l}\text { - DSM-IV-TR } \\
\text { - Spanish version of } \\
\text { the Risk of Suicide } \\
\text { (RS) (Plutchick et } \\
\text { al., 1989) (suicide } \\
\text { risk) }\end{array}$ & $\begin{array}{l}\text { Alcohol, } \\
\text { cocaine, heroin }\end{array}$ & Suicide Risk & $\begin{array}{l}\text { - There was no relationship } \\
\text { between main SUD diagnosis } \\
\text { and risk of suicide. } \\
\text { - Subjects who injected or } \\
\text { nasally administered drugs had } \\
\text { a higher risk of suicide than } \\
\text { those who took drugs orally or } \\
\text { smoked. }\end{array}$ \\
\hline
\end{tabular}




\begin{tabular}{|c|c|c|c|c|c|c|c|}
\hline $\begin{array}{c}\text { Author } \\
\text { (Pub year)/ } \\
\text { Country }\end{array}$ & Study Design & $\begin{array}{l}\text { Overall Sample } \\
\text { (OUD) }\end{array}$ & $\begin{array}{c}\text { Sample } \\
\text { Characteristics }\end{array}$ & $\begin{array}{l}\text { Data Sources \& } \\
\text { Measure(s) }\end{array}$ & $\begin{array}{l}\text { Substances } \\
\text { Examined }\end{array}$ & $\begin{array}{l}\text { Outcomes } \\
\text { (Time frame) }\end{array}$ & Key findings/ Summary \\
\hline $\begin{array}{l}\text { Neale }(2000) / \\
\text { Scotland }\end{array}$ & Mixed-Methods & $77(62)$ & $\begin{array}{l}\text { Individuals with } \\
\text { history of nonfatal } \\
\text { OD. }\end{array}$ & $\begin{array}{l}\text { - Self- } \\
\text { report/Qualitative } \\
\text { interviews }\end{array}$ & $\begin{array}{l}\text { Heroin, alcohol, } \\
\text { temazepam, } \\
\text { diazepam, } \\
\text { methadone }\end{array}$ & $\begin{array}{l}\text { Intentional OD, } \\
\text { accidental OD (lifetime) }\end{array}$ & $\begin{array}{l}\text { - } 49 \% \text { of the } 77 \text { participants } \\
\text { reported suicidal thoughts or } \\
\text { feelings (suicidal intent) prior } \\
\text { to their current OD. } \\
\text { - A significant association was } \\
\text { found between intentional } \\
\text { overdosing and self-reported } \\
\text { history of mental health } \\
\text { problems (p, } 0.001 \text { ). } \\
\text { - Mean number of types of drug } \\
\text { taken prior to OD was } 2.3 \text { for } \\
\text { individuals with an intentional } \\
\text { OD and } 2.1 \text { for accidental ODs. } \\
\text { - Most commonly used drugs } \\
\text { prior to OD were heroin (46 } \\
\text { respondents); alcohol ( } 23 \\
\text { respondents); temazepam (18 } \\
\text { respondents); diazepam (17 } \\
\text { respondents); and methadone } \\
\text { (16 respondents). } \\
\text { - Heroin was the most frequently } \\
\text { used drug by both intentional } \\
\text { (n=16) and accidental ODs } \\
\text { (n=30) but was significantly } \\
\text { more likely to be used in the } \\
\text { accidental than in the } \\
\text { intentional incidents. }\end{array}$ \\
\hline $\begin{array}{l}\text { Olsson et al. } \\
\text { (2016)/Sweden }\end{array}$ & $\begin{array}{l}\text { Prospective } \\
\text { Cohort Study }\end{array}$ & 6,744 (NR) & $\begin{array}{l}\text { Criminal justice } \\
\text { clients with SUDs. }\end{array}$ & $\begin{array}{l}\text { - Addiction Severity } \\
\text { Index } \\
\text { - National Causes of } \\
\text { Death Registry } \\
\text { (Mortality data) } \\
\text { - ICD-10 } \\
\text { (classification of } \\
\text { injury/death) }\end{array}$ & $\begin{array}{l}\text { Alcohol(binge- } \\
\text { drinking), } \\
\text { heroin, other } \\
\text { opioids, } \\
\text { sedatives/tranqui } \\
\text { lizers, cocaine, } \\
\text { amphetamines, } \\
\text { cannabis }\end{array}$ & $\begin{array}{l}\text { SA(s), suicide risk, fatal } \\
\text { accidental intoxication, } \\
\text { injury/intoxication of } \\
\text { undetermined intent }\end{array}$ & $\begin{array}{l}\text { - Cox regression analyses found } \\
\text { death from a fatal accidental } \\
\text { OD was associated with use of } \\
\text { heroin and cannabis; male } \\
\text { gender was marginally } \\
\text { significant. } \\
\text { - Cox regression analysis found } \\
\text { that death from } \\
\text { injury/intoxication of } \\
\text { undetermined intent was } \\
\text { positively associated with use } \\
\text { of heroin, binge drinking } \\
\text { alcohol, and previous } \\
\text { psychiatric hospitalization. } \\
\text { - Sensitivity analysis found that } \\
\text { death from intoxication of } \\
\text { undetermined intent was } \\
\text { positively associated with use } \\
\text { of heroin and binge drinking }\end{array}$ \\
\hline
\end{tabular}




\begin{tabular}{|c|c|c|c|c|c|c|c|}
\hline & & & & & & & $\begin{array}{l}\text { alcohol; negatively associated } \\
\text { with depression. } \\
\text { Death from suicide was } \\
\text { positively associated with } \\
\text { previous SA(s) and use of } \\
\text { sedatives/tranquilizers was } \\
\text { marginally significant. }\end{array}$ \\
\hline $\begin{array}{c}\text { Author } \\
\text { (Pub year)/ } \\
\text { Country }\end{array}$ & Study Design & $\begin{array}{l}\text { Overall Sample } \\
\text { (OUD) }\end{array}$ & $\begin{array}{c}\text { Sample } \\
\text { Characteristics }\end{array}$ & $\begin{array}{c}\text { Data Sources \& } \\
\text { Measure(s) }\end{array}$ & $\begin{array}{l}\text { Substances } \\
\text { Examined }\end{array}$ & $\begin{array}{c}\text { Outcomes } \\
\text { (Time frame) }\end{array}$ & Key findings/ Summary \\
\hline $\begin{array}{l}\text { Oyefeso et al. } \\
\text { (2008)/England }\end{array}$ & Cohort Study & $80(80)$ & $\begin{array}{l}\text { OUD patients } \\
\text { receiving inpatient } \\
\text { and outpatient } \\
\text { treatment. }\end{array}$ & $\begin{array}{l}\text { - ICD-10 } \\
\text { - } 2 \text { items from } \\
\text { Nonadaptive and } \\
\text { Adaptive } \\
\text { Personality (SIB) }\end{array}$ & Opioids & $\begin{array}{l}\text { Patterns and prevalence } \\
\text { of self-injurious } \\
\text { behavior; predictors of } \\
\text { self-injurious behavior } \\
\text { (SIB) (lifetime, past 12- } \\
\text { months) }\end{array}$ & $\begin{array}{l}\text { - } 69 \% \text { of patients reported using } \\
\text { heroin in the past } 30 \text {-days } \\
\text { - } 39 \text { patients endorsed SIB; } \\
\text { lifetime SIB prevalence of } 49 \text {. } \\
\text { - } 10 \text { patients endorsed the } \\
\text { question "Have you hurt } \\
\text { yourself on purpose in the last } \\
12 \text { months?" prior year } \\
\text { prevalence of } 13 \% \text {. } \\
\text { - Reasons for SIB among opiate } \\
\text { addicts was affect-regulation } \\
\text { followed by self-punishment. } \\
\text { - Most frequently reported SIB } \\
\text { types were cutting ( } 74 \% \text { ), nail } \\
\text { biting (51\%), chewing inside } \\
\text { mouth ( } 44 \% \text { ), and self-beating } \\
\text { (36\%). } \\
\text { The most frequently cited } \\
\text { reasons for SIB were to cope } \\
\text { with emotional pain ( } 84 \%), \text { to } \\
\text { express hatred for self ( } 68 \% \text { ), } \\
\text { to express feelings of } \\
\text { helplessness and powerlessness } \\
\text { (63\%) and to stop feelings of } \\
\text { emptiness, numbness and } \\
\text { depression (58\%). } \\
\text { - } 76 \% \text { of patients reported } \\
\text { persistent drug and/or alcohol } \\
\text { problems as a trigged for SIB. }\end{array}$ \\
\hline
\end{tabular}




\begin{tabular}{|c|c|c|c|c|c|c|c|}
\hline $\begin{array}{c}\text { Author } \\
\text { (Pub year)/ } \\
\text { Country }\end{array}$ & Study Design & $\begin{array}{l}\text { Overall Sample } \\
\text { (OUD) }\end{array}$ & $\begin{array}{c}\text { Sample } \\
\text { Characteristics }\end{array}$ & $\begin{array}{c}\text { Data Sources \& } \\
\text { Measure(s) }\end{array}$ & $\begin{array}{l}\text { Substances } \\
\text { Examined }\end{array}$ & $\begin{array}{c}\text { Outcomes } \\
\text { (Time frame) }\end{array}$ & Key findings/ Summary \\
\hline $\begin{array}{l}\text { Pan et al. } \\
\text { (2014)/Taiwan }\end{array}$ & $\begin{array}{l}\text { Cohort Study } \\
\text { (with Nested } \\
\text { Case-Control) }\end{array}$ & $2,750(2,750)$ & $\begin{array}{l}\text { OUD inpatients } \\
\text { admitted between } \\
1990 \text { and } 2010 .\end{array}$ & $\begin{array}{l}\text { - DSM-III \& DSM- } \\
\text { IV (heroin } \\
\text { dependence) } \\
\text { - ICD-9 \& ICD-10 } \\
\text { (suicide mortality) }\end{array}$ & Heroin & $\begin{array}{l}\text { Suicide mortality } \\
\text { (January } 1,1990- \\
\text { December } 31,2010 \text { ) }\end{array}$ & $\begin{array}{l}\text { - Suicides among inpatients with } \\
\text { heroin dependence, had a 16- } \\
\text { fold greater standardized } \\
\text { mortality ratio (SMR=16.2) } \\
\text { compared to the general } \\
\text { population and the highest } \\
\text { SMR among all specified cause } \\
\text { of death categories. } \\
\text { - Mean interval from the index } \\
\text { admission to suicide was } 5.8 \\
\text { years }(\mathrm{SD}=4.2) \text {. } \\
\text { - Methods include: hanging }(\mathrm{n}= \\
\text { 27), charcoal burning/gases } \\
\text { other than in domestic use }(\mathrm{n}= \\
\text { 17),drugs/poisons }(\mathrm{n}=14), \\
\text { jumping from a high place }(\mathrm{n}= \\
\text { 3), drowning ( }=2) \text {, cutting (n } \\
=2) \text {, gases in domestic use (n= }= \\
\text { 1), and others ( }=3 \text { ); no one } \\
\text { used firearms. } \\
\text { - Multivariable analysis found } \\
\text { that SA at index admission and } \\
\text { lifetime depressive syndrome } \\
\text { were significantly associated } \\
\text { with suicide risk. }\end{array}$ \\
\hline $\begin{array}{l}\text { Penney et al. } \\
\text { (2012)/Canada }\end{array}$ & $\begin{array}{l}\text { Case-Control } \\
\text { Study }\end{array}$ & $5,990(\mathrm{NR})$ & $\begin{array}{l}\text { Individuals } \\
\text { seeking addiction } \\
\text { treatment between } \\
2001-2008 \text { with } \\
\text { and without past } \\
\text { year SA. }\end{array}$ & & $\begin{array}{l}\text { Alcohol, } \\
\text { amphetamines, } \\
\text { stimulants, } \\
\text { barbiturates, } \\
\text { benzodiazepines, } \\
\text { cannabis, } \\
\text { cocaine/crack, } \\
\text { ecstasy, glue, } \\
\text { inhalants, } \\
\text { hallucinogens, } \\
\text { opioids }\end{array}$ & $\begin{array}{l}\text { Hospitalized SA(s) (past } \\
12 \text {-months) }\end{array}$ & $\begin{array}{l}\text { - No relationship between } \\
\text { opioids and hospitalization for } \\
\text { SA. }\end{array}$ \\
\hline
\end{tabular}




\begin{tabular}{|c|c|c|c|c|c|c|c|}
\hline $\begin{array}{c}\text { Author } \\
\text { (Pub year)/ } \\
\text { Country }\end{array}$ & Study Design & $\begin{array}{c}\text { Overall Sample } \\
\text { (OUD) }\end{array}$ & $\begin{array}{c}\text { Sample } \\
\text { Characteristics }\end{array}$ & $\begin{array}{c}\text { Data Sources \& } \\
\text { Measure(s) }\end{array}$ & $\begin{array}{l}\text { Substances } \\
\text { Examined }\end{array}$ & $\begin{array}{c}\text { Outcomes } \\
\text { (Time frame) }\end{array}$ & Key findings/ Summary \\
\hline $\begin{array}{l}\text { Ravndal \& } \\
\text { Vaglum } \\
\text { (1999)/Norway }\end{array}$ & $\begin{array}{c}\text { Prospective } \\
\text { Cohort Study }\end{array}$ & $200(112)$ & $\begin{array}{l}\text { Individuals } \\
\text { receiving } \\
\text { treatment for } \\
\text { SUDs between } \\
1986-1988 .\end{array}$ & & $\begin{array}{l}\text { Alcohol, } \\
\text { cannabis, } \\
\text { psychoactive } \\
\text { drugs, opioids, } \\
\text { amphetamines }\end{array}$ & $\begin{array}{l}\text { Prevalence of lifetime } \\
\text { non-fatal ODs and SAs } \\
\text { (baseline, 5-year follow- } \\
\text { up) }\end{array}$ & $\begin{array}{l}\text { - } 43 \%(\mathrm{n}=85) \text { reported being } \\
\text { hospitalized once or several } \\
\text { times because of ODs. } \\
\text { - There was a weak relationship } \\
\text { between OD and SA(s). } \\
\text { - Daily use of opiates and } \\
\text { number of months in inpatient } \\
\text { treatment were significicant } \\
\text { correlates of OD. } \\
\text { - Clients who had spent up to } 1 \\
\text { year altogether in inpatient } \\
\text { treatment had } 4.17 \text { times } \\
\text { greater chance of an OD than } \\
\text { clients who had not been in } \\
\text { treatment } \\
\text { - Clients who had spent } 1 \text { year or } \\
\text { more in inpatient treatment had } \\
8.74 \text { times greater chance of } \\
\text { OD than clients without } \\
\text { inpatient treatment. } \\
\text { - } 47 \% \text { (n=94) of clients reported } \\
\text { having one or more SAs. } \\
\text { - Individuals with SA reported } \\
\text { greater consumption of alcohol } \\
\text { (17 vs. } 13 \text { liters pure alcohol) } \\
\text { and a more frequent use of } \\
\text { opioids ( } 43 \% \text { daily use vs. } \\
32 \% \text { ) in the last } 6 \text {-months. } \\
\text { - Individuals with a SA had more } \\
\text { frequent use of } \\
\text { benzodiazepines, reported more } \\
\text { ODs, had more in- and } \\
\text { outpatient treatment and spent } \\
\text { less time in prison. }\end{array}$ \\
\hline
\end{tabular}




\begin{tabular}{|c|c|c|c|c|c|c|c|}
\hline $\begin{array}{c}\text { Author } \\
\text { (Pub year)/ } \\
\text { Country }\end{array}$ & Study Design & $\begin{array}{c}\text { Overall Sample } \\
\text { (OUD) }\end{array}$ & $\begin{array}{c}\text { Sample } \\
\text { Characteristics }\end{array}$ & $\begin{array}{c}\text { Data Sources \& } \\
\text { Measure(s) }\end{array}$ & $\begin{array}{c}\text { Substances } \\
\text { Examined }\end{array}$ & $\begin{array}{c}\text { Outcomes } \\
\text { (Time frame) }\end{array}$ & Key findings/ Summary \\
\hline $\begin{array}{l}\text { Ross et al. } \\
\text { (2005)/Australia }\end{array}$ & $\begin{array}{l}\text { Prospective } \\
\text { Cohort Study }\end{array}$ & $825(825)$ & $\begin{array}{l}\text { Current heroin } \\
\text { users entering } \\
\text { different treatment } \\
\text { modalities } \\
\text { between February } \\
2001 \text { through } \\
\text { August } 2002 \text {. }\end{array}$ & - DSM-IV & Heroin & $\begin{array}{l}\text { SA(s) (lifetime); OD(s) } \\
\text { (past 12-months; } \\
\text { lifetime) }\end{array}$ & $\begin{array}{l}\text { - } 58 \% \text { of participants had } \\
\text { experienced a heroin OD (OD), } \\
\text { with } 28 \% \text { occurring in the } \\
\text { preceding } 12 \text {-months. } \\
\text { - In the } 12 \text {-months prior to } \\
\text { interview, the RR group was } \\
\text { more likely to have ODd in } \\
\text { than the MT, DTX and NT } \\
\text { groups. } \\
\text { - } 37 \% \text { of the sample reported a } \\
\text { SA, } 14 \% \text { having made an } \\
\text { attempt in the past } 12 \text {-months } \\
\text { and } 6 \% \text { in the preceding month. } \\
\text { - Prevalence of attempted suicide } \\
\text { in the past } 12 \text {-mos did not } \\
\text { differ by treatment modality. } \\
\text { Females were significantly } \\
\text { more likely to have a SA in the } \\
\text { past year. }\end{array}$ \\
\hline $\begin{array}{l}\text { Rossow \& } \\
\text { Lauritzen } \\
\text { (1999)/Norway }\end{array}$ & $\begin{array}{l}\text { Cross Sectional } \\
\text { Study }\end{array}$ & $2,051(1,367)$ & $\begin{array}{l}\text { National sample } \\
\text { of individuals with } \\
\text { SUD seeking } \\
\text { treatment from } \\
\text { 1992-1993. }\end{array}$ & & $\begin{array}{l}\text { Alcohol, } \\
\text { cannabis, } \\
\text { opioids, } \\
\text { amphetamines, } \\
\text { inhalants, } \\
\text { tranquilizers, } \\
\text { other substances }\end{array}$ & $\begin{array}{l}\text { Prevalence of non-fatal } \\
\text { ODs and SA(s); } \\
\text { predictors of covariation } \\
\text { between OD and SA(s) } \\
\text { (lifetime) }\end{array}$ & $\begin{array}{l}\text { - } 45.5 \% \text { reported having } \\
\text { experienced one or more ODs. } \\
\text { - About } 70 \% \text { who experienced an } \\
\text { ODs had two or more } \\
\text { - } 16 \% \text { reported having } 9+\text { ODs } \\
\text { - } 32.7 \% \text { reported having made } \\
\text { one or more SAs, } \\
\text { - Among those who reported } \\
\text { SAs, 56.1\%reported 2+ } \\
\text { attempts. } \\
\text { - Odds of having attempted } \\
\text { suicide were six times higher } \\
\text { among those who had } \\
\text { experienced ODs (OR=6.3 } \\
\text { - The number of SAs was also } \\
\text { positively and significantly } \\
\text { associated with the number of } \\
\text { ODs. } \\
\text { - Individuals with both SAs and } \\
\text { ODs were characterized as } \\
\text { older; more often homeless; } \\
\text { displayed poor social } \\
\text { functioning and mostly } \\
\text { maintaining social relations in } \\
\text { the drug scene; daily use of } \\
\text { opiates, alcohol and } \\
\text { tranquilizers; HIV risk-taking }\end{array}$ \\
\hline
\end{tabular}




\begin{tabular}{|c|c|c|c|c|c|c|c|}
\hline & & & & & & & $\begin{array}{l}\text { behavior, having more } \\
\text { psychiatric problems } \\
\text { (depression and anxiety) and } \\
\text { more severe SI. }\end{array}$ \\
\hline $\begin{array}{c}\text { Author } \\
\text { (Pub year)/ } \\
\text { Country } \\
\end{array}$ & Study Design & $\begin{array}{c}\text { Overall Sample } \\
\text { (OUD) }\end{array}$ & $\begin{array}{c}\text { Sample } \\
\text { Characteristics }\end{array}$ & $\begin{array}{c}\text { Data Sources \& } \\
\text { Measure(s) }\end{array}$ & $\begin{array}{l}\text { Substances } \\
\text { Examined }\end{array}$ & $\begin{array}{c}\text { Outcomes } \\
\text { (Time frame) }\end{array}$ & Key findings/ Summary \\
\hline Roy $(2002) /$ USA & $\begin{array}{l}\text { Case-Control } \\
\text { Study }\end{array}$ & $246(246)$ & $\begin{array}{l}\text { OUD patients with } \\
\text { and without SA. }\end{array}$ & - DSM-IV & $\begin{array}{l}\text { Opioids, alcohol, } \\
\text { cocaine }\end{array}$ & $\begin{array}{l}\text { SA(s), first- and second- } \\
\text { degree relative history of } \\
\text { suicide (lifetime) }\end{array}$ & $\begin{array}{l}\text { - } 105 \text { of the } 246 \text { patients with } \\
\text { OUD reported a lifetime SA. } \\
\text { - } 21 \text { patients attempted suicide } \\
\text { by deliberate heroin OD with } \\
\text { intent to die, and } 12 \text { of those } \\
\text { made at least one additional } \\
\text { attempt using other methods. } \\
\text { - OUD patients with history of } \\
\text { SA had significantly higher } \\
\text { childhood trauma scores for } \\
\text { emotional abuse, physical } \\
\text { abuse, sexual abuse, emotional } \\
\text { neglect, and physical neglect } \\
\text { than compared to OUD patients } \\
\text { without history of suicide. } \\
\text { Significantly more patients with } \\
\text { SA had a history of cocaine } \\
\text { dependence and alcohol } \\
\text { dependence. } \\
\text { - Significantly more people with } \\
\text { SA had history of major } \\
\text { depressive disorder and higher } \\
\text { ASI composite scores. } \\
\text { - Of the } 105 \text { patients with a SA, } \\
31 \text { had a family history of } \\
\text { suicidal behavior. } \\
\text { - Multivariable analysis also } \\
\text { found that neuroticism with } \\
\text { significantly and independently } \\
\text { associated with SA and had } \\
\text { higher hostility scores. }\end{array}$ \\
\hline
\end{tabular}




\begin{tabular}{|c|c|c|c|c|c|c|c|}
\hline $\begin{array}{c}\text { Author } \\
\text { (Pub year)/ } \\
\text { Country }\end{array}$ & Study Design & $\begin{array}{l}\text { Overall Sample } \\
\text { (OUD) }\end{array}$ & $\begin{array}{c}\text { Sample } \\
\text { Characteristics }\end{array}$ & $\begin{array}{c}\text { Data Sources \& } \\
\text { Measure(s) }\end{array}$ & $\begin{array}{l}\text { Substances } \\
\text { Examined }\end{array}$ & $\begin{array}{c}\text { Outcomes } \\
\text { (Time frame) }\end{array}$ & Key findings/ Summary \\
\hline Roy $(2010) /$ USA & $\begin{array}{l}\text { Case-Control } \\
\text { Study }\end{array}$ & $527(527)$ & $\begin{array}{l}\text { Abstinent OUD } \\
\text { patients. }\end{array}$ & & $\begin{array}{l}\text { Opioids, alcohol, } \\
\text { cocaine }\end{array}$ & $\begin{array}{l}\text { SA(s) \& familial history } \\
\text { of suicide (lifetime) }\end{array}$ & $\begin{array}{l}\text { - } 207 \text { of the } 527 \text { OUD patients } \\
(39.3 \%) \text { attempted suicide. } \\
\text { - Patients who had attempted } \\
\text { were younger than those who } \\
\text { had no history of SA and a } \\
\text { majority were female. } \\
\text { - Significant predictors of SA } \\
\text { were family history of suicidal } \\
\text { behavior, alcohol or cocaine } \\
\text { dependence, and receiving } \\
\text { antidepressant medication. }\end{array}$ \\
\hline $\begin{array}{l}\text { Tremeau et al. } \\
(2005) / \text { France }\end{array}$ & $\begin{array}{l}\text { Case-Control } \\
\text { Study }\end{array}$ & $160(160)$ & $\begin{array}{l}\text { OUD patients } \\
\text { receiving } \\
\text { methadone or } \\
\text { buprenorphine } \\
\text { treatment. }\end{array}$ & $\begin{array}{l}\text { - DSM-IV } \\
\text { - Urine toxicology } \\
\text { (confirm opioid } \\
\text { use at intake) }\end{array}$ & Opioids & $\begin{array}{l}\text { Characteristics of SA(s), } \\
\text { familial history of SA }\end{array}$ & $\begin{array}{l}\text { - } 27 \% \text { of patients ( } 10 \text { men and } \\
11 \text { women) made their first SA } \\
\text { before they experienced } \\
\text { heroin. } \\
\text { - Women made more attempts } \\
\text { and they resorted to non- } \\
\text { heroin drug ODs more } \\
\text { frequently. }\end{array}$ \\
\hline $\begin{array}{l}\text { Vingoe et al. } \\
\text { (1999)/England }\end{array}$ & $\begin{array}{l}\text { Cross Sectional } \\
\text { Study }\end{array}$ & $48(48)$ & $\begin{array}{l}\text { Outpatients } \\
\text { receiving } \\
\text { treatment for } \\
\text { SUDs, with a } \\
\text { history of opioid } \\
\text { IDU. }\end{array}$ & & Heroin & $\begin{array}{l}\text { Non-intentional OD(s); } \\
\text { intentional OD(s) or } \\
\text { SA(s) (lifetime, past 12- } \\
\text { months) }\end{array}$ & $\begin{array}{l}\text { - } 54 \%(\mathrm{n}=26) \text { of patients had } \\
\text { experienced a heroin OD in the } \\
\text { past, } 10 \text { had only overdosed } \\
\text { once; } 16 \text { had multiple ODs. } \\
\text { - } 60 \%(\mathrm{n}=29) \text { patients had a } \\
\text { history of suicidal thoughts } \\
\text { - } 26 \text { had a suicide plan; plans } \\
\text { include: } 6 \text { had thoughts about } \\
\text { heroin OD, } 14 \text { had considered } \\
\text { OD of other drugs, } 3 \text { had } \\
\text { thought of cutting, } 2 \text { of } \\
\text { shooting and } 1 \text { of jumping. } \\
\text { - } 35 \% \text { (n=17) patients had made } \\
\text { SAs in the past. Methods of } \\
\text { attempt include: } 2 \text { took a heroin } \\
\text { OD, } 12 \text { took an OD of other } \\
\text { drugs, } 2 \text { by cutting, and } 1 \text { by } \\
\text { jumping. } \\
\text { - History of heroin OD, half } \\
\text { (13/26) made a SA at some } \\
\text { time in their lives. } \\
\text { - OD of heroin was not a } \\
\text { common method for attempting } \\
\text { suicide: of the } 13 \text { subjects who } \\
\text { had a history of both heroin OD } \\
\text { and of a SA, } 2 \text { used intentional } \\
\text { heroin OD. }\end{array}$ \\
\hline
\end{tabular}




\begin{tabular}{|c|c|c|c|c|c|c|c|}
\hline $\begin{array}{c}\text { Author } \\
\text { (Pub year)/ } \\
\text { Country }\end{array}$ & Study Design & $\begin{array}{c}\text { Overall Sample } \\
\text { (OUD) }\end{array}$ & $\begin{array}{c}\text { Sample } \\
\text { Characteristics }\end{array}$ & $\begin{array}{c}\text { Data Sources \& } \\
\text { Measure(s) }\end{array}$ & $\begin{array}{l}\text { Substances } \\
\text { Examined }\end{array}$ & $\begin{array}{c}\text { Outcomes } \\
\text { (Time frame) }\end{array}$ & Key findings/ Summary \\
\hline $\begin{array}{l}\text { Wines et al. } \\
(2004) / \text { USA }\end{array}$ & $\begin{array}{l}\text { Cohort Study } \\
\text { (Prospective) }\end{array}$ & $470(470)$ & $\begin{array}{l}\text { Adults admitted to } \\
\text { an inpatient } \\
\text { treatment. }\end{array}$ & $\begin{array}{l}\text { - 2-questions from } \\
\text { ASI (suicidal } \\
\text { behavior) }\end{array}$ & $\begin{array}{l}\text { Alcohol, heroin, } \\
\text { benzodiazepines, } \\
\text { cocaine, } \\
\text { sedatives, } \\
\text { stimulants }\end{array}$ & $\begin{array}{l}\text { SI, SA(s) }(6,12,18, \& \\
24 \text {-months, lifetime) }\end{array}$ & $\begin{array}{l}\text { - No significant associations } \\
\text { between frequency of recent } \\
\text { alcohol, heroin, } \\
\text { benzodiazepines or cocaine use } \\
\text { and SAs, whether or not } \\
\text { depressive symptoms were } \\
\text { present } \\
\text { - Alcohol, heroin, } \\
\text { benzodiazepines or cocaine } \\
\text { were not significantly } \\
\text { associated with either SI or SA. }\end{array}$ \\
\hline
\end{tabular}




\subsection{A). Evaluation of Case-Control Studies}

\begin{tabular}{|c|c|c|c|c|c|c|c|c|c|c|c|c|c|c|}
\hline $\begin{array}{c}\text { Author } \\
\text { (Pub year) }\end{array}$ & Study Design & CL1 & CL2 & CL3 & CL4 & CL5 & CL6 & CL7 & CL8 & CL9 & CL10 & CL11 & CL12 & $\begin{array}{c}\text { Quality } \\
\text { Rating }\end{array}$ \\
\hline $\begin{array}{l}\text { Dragisic } \\
(2015)\end{array}$ & Case-Control & $\mathrm{Y}$ & $\mathrm{N}$ & $\mathrm{N}$ & $\mathrm{Y}$ & $\mathrm{N}$ & $\mathrm{Y}$ & NA & $\mathrm{Y}$ & $\mathrm{Y}$ & $\mathrm{Y}$ & $\mathrm{N}$ & $\mathrm{N}$ & Poor \\
\hline $\begin{array}{l}\text { Ghaffari- } \\
\text { Nejad } \\
(2011)\end{array}$ & Case-Control & $\mathrm{Y}$ & $\mathrm{N}$ & $\mathrm{Y}$ & $\mathrm{Y}$ & $\mathrm{N}$ & $\mathrm{N}$ & $\mathrm{N}$ & $\mathrm{Y}$ & $\mathrm{Y}$ & $\mathrm{Y}$ & NR & $\mathrm{Y}$ & Poor \\
\hline $\begin{array}{l}\text { Jin } \\
(2013) \\
\end{array}$ & Case-Control & $\mathrm{Y}$ & $\mathrm{Y}$ & $\mathrm{N}$ & $\mathrm{Y}$ & $\bar{Y}$ & $\bar{Y}$ & $\mathrm{NR}$ & $\mathrm{N}$ & $\bar{Y}$ & $\bar{Y}$ & NR & $\mathrm{Y}$ & Good \\
\hline $\begin{array}{l}\text { Ilgen } \\
(2009)\end{array}$ & Case-Control & $\mathrm{Y}$ & $\bar{Y}$ & $\mathrm{~N}$ & $\bar{Y}$ & $\bar{Y}$ & $\bar{Y}$ & $\bar{Y}$ & $\mathrm{~N}$ & $\bar{Y}$ & $\bar{Y}$ & NR & $\bar{Y}$ & Good \\
\hline $\begin{array}{l}\text { Kazour } \\
(2016)\end{array}$ & Case-Control & $\mathrm{Y}$ & $\mathrm{Y}$ & $\mathrm{N}$ & $\mathrm{Y}$ & $\begin{array}{l}\mathrm{Y} \\
\end{array}$ & Y & NR & $\mathrm{N}$ & $\mathrm{Y}$ & $\mathrm{Y}$ & NR & $\mathrm{Y}$ & Good \\
\hline $\begin{array}{l}\text { Maloney } \\
(2009)\end{array}$ & Case-Control & $\mathrm{Y}$ & $\mathrm{Y}$ & $\mathrm{Y}$ & $\mathrm{Y}$ & $\mathrm{Y}$ & $\mathrm{Y}$ & NR & $\mathrm{N}$ & $\mathrm{Y}$ & $\mathrm{Y}$ & $\mathrm{Y}$ & $\mathrm{Y}$ & Good \\
\hline $\begin{array}{l}\text { Maloney } \\
(2010)\end{array}$ & Case-Control & $\mathrm{Y}$ & $\mathrm{Y}$ & $\mathrm{Y}$ & $\mathrm{Y}$ & $\mathrm{Y}$ & $\mathrm{Y}$ & $\mathrm{NR}$ & $\mathrm{N}$ & $\mathrm{Y}$ & $\mathrm{Y}$ & NR & $\mathrm{Y}$ & Good \\
\hline $\begin{array}{l}\text { Penney } \\
(2012)\end{array}$ & Case-Control & $\mathrm{Y}$ & $\mathrm{Y}$ & $\mathrm{N}$ & $\mathrm{Y}$ & $\mathrm{Y}$ & $\mathrm{Y}$ & NA & NA & NA & $\mathrm{Y}$ & $\mathrm{N}$ & $\mathrm{Y}$ & Fair \\
\hline $\begin{array}{l}\text { Roy } \\
(2002)\end{array}$ & Case-Control & $\mathrm{Y}$ & $\bar{Y}$ & $\mathrm{~N}$ & $\bar{Y}$ & $\bar{Y}$ & $\mathrm{~N}$ & NA & $\mathrm{N}$ & NA & $\bar{Y}$ & NR & $\bar{Y}$ & Fair \\
\hline $\begin{array}{l}\text { Roy } \\
(2010)\end{array}$ & Case-Control & $\mathrm{Y}$ & $\mathrm{Y}$ & $\mathrm{N}$ & $\mathrm{Y}$ & $\mathrm{Y}$ & $\mathrm{Y}$ & $\mathrm{NR}$ & NR & $\mathrm{N}$ & $\mathrm{Y}$ & NR & $\mathrm{Y}$ & Good \\
\hline $\begin{array}{l}\text { Tremeau } \\
(2005)\end{array}$ & Case-Control & $\mathrm{Y}$ & $\bar{Y}$ & $\mathrm{~N}$ & $\mathrm{Y}$ & $\bar{Y}$ & $\mathrm{~N}$ & NR & NR & $\mathrm{Y}$ & $\mathrm{Y}$ & $\mathrm{N}$ & $\bar{Y}$ & Good \\
\hline
\end{tabular}

Abbreviation definitions: $\mathrm{Y}=\mathrm{Yes} ; \mathrm{N}=\mathrm{No} ; \mathrm{NR}=$ Not Reported; $\mathrm{NA}=$ Not Applicable; $\mathrm{CD}=$ Cannot Determine; $\mathrm{CL}=\mathrm{CheCLlist}$ Item Key for Case-Control Studies:

CL1. Was the research question or objective in this paper clearly stated?

CL 2. Was the study population clearly specified and defined?

CL 3. Was the participation rate of eligible persons at least $50 \%$ ?

CL 4. Were all the subjects selected or recruited from the same or similar populations (including the same time period)? Were inclusion and exclusion criteria for being in the study prespecified and applied

uniformly to all

participants?

CL 5. Was a sample size justification, power description, or variance and effect estimates provided?

CL 6. For the analyses in this paper, were the exposure(s) of interest measured prior to the outcome(s) being measured?

CL 7. Was the timeframe sufficient so that one could reasonably expect to see an association between exposure and outcome if it existed?

CL 8. For exposures that can vary in amount or level, did the study examine different levels of the exposure as related to the outcome (e.g., categories of exposure, or exposure measured as continuous variable)?

CL 9. Were the exposure measures (independent variables) clearly defined, valid, reliable, and implemented consistently across all study participants?

CL 10. Was the exposure(s) assessed more than once over time?

CL 11. Were the outcome measures (dependent variables) clearly defined, valid, reliable, and implemented consistently across all study participants?

CL 12. Were the outcome assessors blinded to the exposure status of participants? 


\subsection{B). Evaluation of Cohort and Cross-Sectional Studies}

\begin{tabular}{|c|c|c|c|c|c|c|c|c|c|c|c|c|c|c|c|c|}
\hline $\begin{array}{c}\text { Author } \\
\text { (Pub year) }\end{array}$ & Study Design & CL1 & CL2 & CL3 & CL4 & CL5 & CL6 & CL7 & CL8 & CL9 & CL10 & CL11 & CL12 & CL13 & CL14 & $\begin{array}{l}\text { Quality } \\
\text { Rating }\end{array}$ \\
\hline $\begin{array}{l}\text { Ashrafioun } \\
\text { (2017) }\end{array}$ & Cross Sectional & $\mathrm{Y}$ & $\mathrm{Y}$ & $\mathrm{Y}$ & $\mathrm{Y}$ & $\mathrm{Y}$ & $\mathrm{N}$ & $\mathrm{N}$ & $\mathrm{Y}$ & $\mathrm{Y}$ & $\mathrm{N}$ & $\mathrm{Y}$ & NA & NA & $\mathrm{Y}$ & Good \\
\hline $\begin{array}{l}\text { Baykara } \\
(2018)\end{array}$ & Cross Sectional & $\mathrm{Y}$ & $\mathrm{Y}$ & NR & $\bar{Y}$ & $\mathrm{~N}$ & $\mathrm{~N}$ & $\mathrm{~N}$ & $\mathrm{~N}$ & $\mathrm{Y}$ & $\mathrm{N}$ & $\mathrm{Y}$ & $\mathrm{N}$ & NA & $\mathrm{N}$ & Poor \\
\hline $\begin{array}{l}\text { Best } \\
(2000)\end{array}$ & Cross Sectional & $\bar{Y}$ & $\mathrm{Y}$ & NA & $\mathrm{Y}$ & $\mathrm{N}$ & $\mathrm{N}$ & $\mathrm{N}$ & $\mathrm{Y}$ & $\mathrm{Y}$ & $\mathrm{N}$ & $\bar{Y}$ & $\mathrm{Y}$ & NA & $\mathrm{N}$ & Fair \\
\hline $\begin{array}{l}\text { Bohnert } \\
\text { (2017) }\end{array}$ & $\begin{array}{l}\text { Cohort Study } \\
\text { (Retrospective) }\end{array}$ & $\mathrm{Y}$ & $\mathrm{Y}$ & $\mathrm{Y}$ & $\mathrm{Y}$ & $\mathrm{Y}$ & $\mathrm{Y}$ & $\mathrm{Y}$ & $\mathrm{N}$ & $\mathrm{Y}$ & NA & $\bar{Y}$ & $\mathrm{~N}$ & NA & $\mathrm{Y}$ & Good \\
\hline $\begin{array}{l}\text { Borges } \\
(2000)\end{array}$ & Cross Sectional & $\bar{Y}$ & $\mathrm{Y}$ & $\mathrm{Y}$ & $\mathrm{Y}$ & $\mathrm{Y}$ & $\mathrm{N}$ & $\mathrm{N}$ & $\mathrm{N}$ & $\mathrm{Y}$ & $\mathrm{N}$ & $\mathrm{Y}$ & $\mathrm{N}$ & NA & $\mathrm{Y}$ & Fair \\
\hline $\begin{array}{l}\text { Bradvik } \\
(2007)\end{array}$ & Cross Sectional & $\bar{Y}$ & $\mathrm{Y}$ & $\bar{Y}$ & $\mathrm{~N}$ & $\mathrm{~N}$ & $\mathrm{~N}$ & $\mathrm{~N}$ & $\mathrm{~N}$ & $\mathrm{Y}$ & $\mathrm{N}$ & $\mathrm{Y}$ & $\mathrm{N}$ & NA & $\mathrm{N}$ & Poor \\
\hline $\begin{array}{l}\text { Cheek } \\
(2016)\end{array}$ & Cross Sectional & $\mathrm{Y}$ & $\mathrm{Y}$ & $\bar{Y}$ & $\mathrm{Y}$ & $\mathrm{Y}$ & $\mathrm{N}$ & $\mathrm{N}$ & $\mathrm{N}$ & $\mathrm{Y}$ & $\mathrm{N}$ & $\mathrm{Y}$ & $\mathrm{N}$ & NA & $\mathrm{Y}$ & Good \\
\hline $\begin{array}{l}\text { Chen } \\
\text { (2009) }\end{array}$ & Cross Sectional & $\mathrm{Y}$ & $\mathrm{Y}$ & $\mathrm{CD}$ & $\mathrm{Y}$ & $\mathrm{N}$ & $\mathrm{N}$ & $\mathrm{N}$ & $\mathrm{N}$ & $\mathrm{Y}$ & $\mathrm{Y}$ & $\mathrm{Y}$ & $\mathrm{N}$ & NA & $\mathrm{Y}$ & Fair \\
\hline $\begin{array}{l}\text { Conner } \\
(2007)\end{array}$ & $\begin{array}{l}\text { Cohort } \\
\text { (Prospective) }\end{array}$ & $\mathrm{Y}$ & $\mathrm{Y}$ & $\mathrm{CD}$ & $\mathrm{Y}$ & $\mathrm{N}$ & $\mathrm{N}$ & $\mathrm{N}$ & $\mathrm{N}$ & $\mathrm{Y}$ & $\mathrm{N}$ & $\mathrm{Y}$ & $\mathrm{N}$ & $\mathrm{N}$ & $\mathrm{Y}$ & Fair \\
\hline $\begin{array}{l}\text { Darke } \\
(2001)\end{array}$ & Cross Sectional & $\bar{Y}$ & $\bar{Y}$ & $\mathrm{CD}$ & $\mathrm{Y}$ & $\mathrm{N}$ & $\mathrm{N}$ & $\mathrm{N}$ & $\mathrm{Y}$ & $\mathrm{Y}$ & $\mathrm{N}$ & $\mathrm{Y}$ & $\mathrm{N}$ & NA & $\bar{Y}$ & Fair \\
\hline $\begin{array}{l}\text { Darke } \\
(2004)\end{array}$ & Cross Sectional & $\mathrm{Y}$ & $\mathrm{Y}$ & $\bar{Y}$ & $\bar{Y}$ & $\mathrm{~N}$ & $\mathrm{~N}$ & $\mathrm{~N}$ & $\mathrm{Y}$ & $\mathrm{Y}$ & $\bar{Y}$ & $\mathrm{Y}$ & $\mathrm{N}$ & NA & $\bar{Y}$ & Good \\
\hline $\begin{array}{l}\text { Darke } \\
(2010)\end{array}$ & Cross Sectional & $\bar{Y}$ & $\mathrm{Y}$ & $\mathrm{CD}$ & $\mathrm{Y}$ & $\mathrm{N}$ & $\mathrm{N}$ & $\mathrm{N}$ & $\mathrm{Y}$ & $\mathrm{N}$ & $\mathrm{Y}$ & $\mathrm{Y}$ & $\mathrm{N}$ & NA & $\mathrm{Y}$ & Good \\
\hline $\begin{array}{l}\text { Darke } \\
(2012)\end{array}$ & Cross Sectional & $\mathrm{Y}$ & $\mathrm{Y}$ & $\mathrm{CD}$ & $\mathrm{Y}$ & $\mathrm{N}$ & $\mathrm{N}$ & $\mathrm{N}$ & $\mathrm{N}$ & $\mathrm{Y}$ & $\mathrm{N}$ & $\mathrm{Y}$ & $\mathrm{N}$ & NA & $\mathrm{Y}$ & Fair \\
\hline $\begin{array}{l}\text { Darke } \\
(2015)\end{array}$ & $\begin{array}{l}\text { Cohort } \\
\text { (Prospective) }\end{array}$ & $\mathrm{Y}$ & $\mathrm{Y}$ & $\mathrm{Y}$ & $\mathrm{Y}$ & $\mathrm{N}$ & $\mathrm{Y}$ & $\mathrm{Y}$ & $\mathrm{Y}$ & $\mathrm{Y}$ & $\mathrm{Y}$ & $\mathrm{Y}$ & $\mathrm{N}$ & $\mathrm{N}$ & $\mathrm{Y}$ & Good \\
\hline
\end{tabular}

2.2B). Evaluation of Cohort and Cross-Sectional Studies (Continued)

\begin{tabular}{|c|c|c|c|c|c|c|c|c|c|c|c|c|c|c|c|c|}
\hline $\begin{array}{l}\text { Author } \\
\text { (Pub year) }\end{array}$ & Study Design & CL1 & CL2 & CL3 & CL4 & CL5 & CL6 & CL7 & CL8 & CL9 & CL10 & CL11 & CL12 & CL13 & CL14 & $\begin{array}{l}\text { Quality } \\
\text { Rating }\end{array}$ \\
\hline $\begin{array}{l}\text { de los } \\
\text { Cobos } \\
(2007)\end{array}$ & Cross Sectional & $\bar{Y}$ & $\mathrm{~N}$ & $\mathrm{CD}$ & $\bar{Y}$ & $\mathrm{~N}$ & $\mathrm{~N}$ & $\mathrm{~N}$ & $\mathrm{~N}$ & $\bar{Y}$ & $\mathrm{~N}$ & $\bar{Y}$ & $\bar{Y}$ & NA & $\bar{Y}$ & Fair \\
\hline $\begin{array}{l}\text { Gilmore } \\
(2018)\end{array}$ & Cross Sectional & $\bar{Y}$ & $\bar{Y}$ & NR & $\mathrm{Y}$ & $\mathrm{N}$ & $\mathrm{N}$ & $\mathrm{N}$ & $\mathrm{N}$ & $\bar{Y}$ & $\mathrm{~N}$ & $\mathrm{Y}$ & $\mathrm{N}$ & NA & $\bar{Y}$ & Good \\
\hline $\begin{array}{l}\text { Hakansson } \\
(2010)\end{array}$ & Cross Sectional & $\mathrm{Y}$ & $\mathrm{Y}$ & $\mathrm{N}$ & $\mathrm{Y}$ & $\mathrm{Y}$ & $\mathrm{N}$ & $\mathrm{N}$ & NA & $\mathrm{N}$ & $\mathrm{N}$ & $\mathrm{Y}$ & $\mathrm{N}$ & $\mathrm{Y}$ & $\mathrm{N}$ & Good \\
\hline $\begin{array}{l}\begin{array}{l}\text { Harned } \\
(2006)\end{array} \\
\end{array}$ & Cross Sectional & $\mathrm{Y}$ & $\mathrm{Y}$ & NR & $\mathrm{Y}$ & $\mathrm{N}$ & $\mathrm{N}$ & $\mathrm{N}$ & $\mathrm{N}$ & $\mathrm{Y}$ & $\mathrm{N}$ & $\mathrm{Y}$ & NR & NA & $\mathrm{N}$ & Poor \\
\hline
\end{tabular}


2.2B). Evaluation of Cohort and Cross-Sectional Studies (Continued)

\begin{tabular}{|c|c|c|c|c|c|c|c|c|c|c|c|c|c|c|c|c|}
\hline $\begin{array}{l}\text { Author } \\
\text { (Pub year) }\end{array}$ & Study Design & CL1 & CL2 & CL3 & CL4 & CL5 & CL6 & CL7 & CL8 & CL9 & CL10 & CL11 & CL12 & CL13 & CL14 & $\begin{array}{l}\text { Quality } \\
\text { Rating }\end{array}$ \\
\hline $\begin{array}{l}\text { Havens } \\
(2004)\end{array}$ & Cross Sectional & $\mathrm{Y}$ & $\mathrm{Y}$ & $\mathrm{CD}$ & $\mathrm{Y}$ & $\mathrm{N}$ & $\mathrm{N}$ & $\mathrm{N}$ & $\mathrm{Y}$ & $\mathrm{Y}$ & $\mathrm{N}$ & $\mathrm{N}$ & $\mathrm{N}$ & NA & $\mathrm{Y}$ & Good \\
\hline $\begin{array}{l}\text { Icick } \\
(2017)\end{array}$ & Cross Sectional & $\mathrm{Y}$ & $\mathrm{Y}$ & $\mathrm{CD}$ & $\mathrm{Y}$ & $\mathrm{N}$ & $\mathrm{Y}$ & $\mathrm{Y}$ & $\mathrm{Y}$ & $\mathrm{Y}$ & $\mathrm{N}$ & $\mathrm{Y}$ & $\mathrm{CD}$ & NA & $\mathrm{Y}$ & Good \\
\hline $\begin{array}{l}\text { Kalyoncu } \\
(2007)\end{array}$ & Cross Sectional & $\mathrm{Y}$ & $\mathrm{N}$ & $\mathrm{CD}$ & $\mathrm{CD}$ & $\mathrm{N}$ & $\mathrm{N}$ & $\mathrm{N}$ & $\mathrm{N}$ & $\mathrm{Y}$ & $\mathrm{N}$ & $\mathrm{Y}$ & NR & NA & $\mathrm{Y}$ & Poor \\
\hline $\begin{array}{l}\text { Kuramoto } \\
(2012)\end{array}$ & Cross Sectional & $\bar{Y}$ & $\bar{Y}$ & $\bar{Y}$ & $\mathrm{Y}$ & NA & $\mathrm{N}$ & $\mathrm{N}$ & $\mathrm{Y}$ & $\bar{Y}$ & $\mathrm{~N}$ & $\mathrm{Y}$ & $\mathrm{N}$ & NA & $\mathrm{Y}$ & Good \\
\hline $\begin{array}{l}\text { Lavania } \\
(2012)\end{array}$ & Cross Sectional & $\mathrm{Y}$ & $\mathrm{Y}$ & $\mathrm{CD}$ & $\mathrm{Y}$ & $\mathrm{Y}$ & $\mathrm{N}$ & $\mathrm{N}$ & $\mathrm{N}$ & $\mathrm{Y}$ & $\mathrm{N}$ & $\mathrm{Y}$ & $\mathrm{CD}$ & NA & $\bar{Y}$ & Good \\
\hline $\begin{array}{l}\text { Marchand } \\
(2017)\end{array}$ & Cross Sectional & $\bar{Y}$ & $\bar{Y}$ & $\mathrm{CD}$ & $\mathrm{Y}$ & $\mathrm{N}$ & $\mathrm{N}$ & $\mathrm{N}$ & $\mathrm{N}$ & $\bar{Y}$ & $\mathrm{~N}$ & $\bar{Y}$ & $\mathrm{CD}$ & NA & $\bar{Y}$ & Fair \\
\hline $\begin{array}{l}\text { Maremman } \\
\text { i (2007) }\end{array}$ & Cross Sectional & $\mathrm{Y}$ & $\mathrm{Y}$ & NR & NR & $\mathrm{N}$ & $\mathrm{N}$ & $\mathrm{N}$ & $\mathrm{N}$ & $\mathrm{Y}$ & $\mathrm{N}$ & $\mathrm{Y}$ & NR & NA & $\mathrm{CD}$ & Poor \\
\hline $\begin{array}{l}\text { Masferrer } \\
(2016)\end{array}$ & Cross Sectional & $\bar{Y}$ & $\bar{Y}$ & $\mathrm{Y}$ & $\mathrm{Y}$ & $\bar{Y}$ & $\mathrm{~N}$ & $\mathrm{~N}$ & $\mathrm{~N}$ & $\bar{Y}$ & $\mathrm{~N}$ & $\bar{Y}$ & NR & NA & $\bar{Y}$ & Good \\
\hline $\begin{array}{l}\text { Olsson } \\
(2016)\end{array}$ & Cohort & Y & $\mathrm{Y}$ & $\mathrm{Y}$ & $\mathrm{Y}$ & NA & $\mathrm{Y}$ & $\mathrm{Y}$ & NA & $\mathrm{Y}$ & $\mathrm{N}$ & Y & $\mathrm{N}$ & $\mathrm{Y}$ & $\mathrm{Y}$ & Good \\
\hline $\begin{array}{l}\text { Oyefeso } \\
(2008)\end{array}$ & Cohort & $\mathrm{Y}$ & $\mathrm{Y}$ & $\mathrm{Y}$ & $\mathrm{Y}$ & NR & $\mathrm{Y}$ & $\mathrm{Y}$ & $\mathrm{N}$ & $\mathrm{Y}$ & $\mathrm{N}$ & $\mathrm{Y}$ & NR & NA & $\mathrm{CD}$ & Fair \\
\hline $\begin{array}{l}\text { Pan } \\
(2014)\end{array}$ & $\begin{array}{l}\text { Cohort (Nested } \\
\text { Case-Control) }\end{array}$ & $\mathrm{Y}$ & $\mathrm{Y}$ & $\mathrm{CD}$ & $\mathrm{Y}$ & NA & $\mathrm{Y}$ & $\mathrm{Y}$ & $\mathrm{N}$ & $\mathrm{Y}$ & $\mathrm{N}$ & $\mathrm{Y}$ & $\mathrm{N}$ & NA & $\mathrm{Y}$ & Good \\
\hline $\begin{array}{l}\text { Ravndal } \\
(1999)\end{array}$ & Cohort & $\mathrm{Y}$ & $\mathrm{Y}$ & NR & $\mathrm{Y}$ & $\mathrm{N}$ & $\mathrm{Y}$ & $\mathrm{Y}$ & $\mathrm{N}$ & $\mathrm{Y}$ & $\mathrm{N}$ & $\mathrm{Y}$ & NR & $\mathrm{N}$ & $\mathrm{CD}$ & Fair \\
\hline $\begin{array}{l}\text { Ross } \\
(2005)\end{array}$ & Cohort Study & $\mathrm{Y}$ & $\mathrm{Y}$ & NR & $\mathrm{Y}$ & $\mathrm{N}$ & $\bar{Y}$ & $\mathrm{Y}$ & $\mathrm{Y}$ & $\bar{Y}$ & $\bar{Y}$ & $\bar{Y}$ & $\mathrm{CD}$ & NR & NR & Good \\
\hline $\begin{array}{l}\text { Rossow } \\
(1999)\end{array}$ & Cross Sectional & $\mathrm{Y}$ & $\mathrm{Y}$ & $\mathrm{N}$ & $\mathrm{Y}$ & $\mathrm{CD}$ & $\mathrm{N}$ & $\mathrm{N}$ & $\mathrm{N}$ & $\mathrm{Y}$ & $\mathrm{Y}$ & $\mathrm{N}$ & $\mathrm{Y}$ & NR & NA & Fair \\
\hline $\begin{array}{l}\text { Vingoe } \\
(1999)\end{array}$ & Cross Sectional & $\bar{Y}$ & $\mathrm{~N}$ & NR & $\mathrm{CD}$ & $\mathrm{N}$ & $\mathrm{N}$ & $\mathrm{N}$ & $\mathrm{CD}$ & $\bar{Y}$ & $\mathrm{~N}$ & $\bar{Y}$ & NR & NA & $\mathrm{N}$ & Poor \\
\hline
\end{tabular}


2.2B). Evaluation of Cohort and Cross-Sectional Studies (Continued)

\begin{tabular}{|c|c|c|c|c|c|c|c|c|c|c|c|c|c|c|c|c|}
\hline $\begin{array}{l}\text { Author } \\
\text { (Pub year) }\end{array}$ & Study Design & CL1 & CL2 & CL3 & CL4 & CL5 & CL6 & CL7 & CL8 & CL9 & CL10 & CL11 & CL12 & CL13 & CL14 & $\begin{array}{l}\text { Quality } \\
\text { Rating }\end{array}$ \\
\hline $\begin{array}{l}\text { Wines } \\
(2004)\end{array}$ & Cohort & $\mathrm{Y}$ & $\mathrm{Y}$ & NR & $\mathrm{Y}$ & $\mathrm{N}$ & $\mathrm{Y}$ & $\mathrm{Y}$ & $\mathrm{N}$ & $\mathrm{Y}$ & $\mathrm{N}$ & $\mathrm{Y}$ & $\mathrm{N}$ & NR & $\mathrm{Y}$ & Fair \\
\hline $\begin{array}{l}\text { Neale } \\
(2000)\end{array}$ & Mixed Methods & - & - & - & - & - & & - & - & - & - & - & - & - & - & N/A \\
\hline
\end{tabular}

Abbreviation definitions: $\mathrm{Y}=\mathrm{Yes} ; \mathrm{N}=\mathrm{No} ; \mathrm{NR}=$ Not Reported; $\mathrm{NA}=$ Not Applicable; $\mathrm{CD}=$ Cannot Determine; $\mathrm{CL}=\mathrm{CheCLlist}$ Item

\section{Key for Cross Sectional/Cohort Studies:}

CL1. Was the research question or objective in this paper clearly stated?

CL 2. Was the study population clearly specified and defined?

CL 3. Was the participation rate of eligible persons at least $50 \%$ ?

CL 4. Were all the subjects selected or recruited from the same or similar populations (including the same time period)? Were inclusion and exclusion criteria for being in the study prespecified and applied uniformly to all

participants?

CL 5. Was a sample size justification, power description, or variance and effect estimates provided?

CL 6. For the analyses in this paper, were the exposure(s) of interest measured prior to the outcome(s) being measured?

CL 7. Was the timeframe sufficient so that one could reasonably expect to see an association between exposure and outcome if it existed?

CL 8. For exposures that can vary in amount or level, did the study examine different levels of the exposure as related to the outcome (e.g., categories of exposure, or exposure measured as continuous variable)?

CL 9. Were the exposure measures (independent variables) clearly defined, valid, reliable, and implemented consistently across all study participants?

CL 10. Was the exposure(s) assessed more than once over time?

CL 11. Were the outcome measures (dependent variables) clearly defined, valid, reliable, and implemented consistently across all study participants?

CL 12. Were the outcome assessors blinded to the exposure status of participants?

CL 13. Was loss to follow-up after baseline $20 \%$ or less?

CL 14. Were key potential confounding variables measured and adjusted statistically for their impact on the relationship between exposure(s) and outcome(s)? 


\section{Chapter 3}

\section{Feasibility and Acceptability of Safety Screening Among Patients Receiving Addiction Treatment}

\subsection{Abstract}

Background: Premature mortality associated with opioid-related overdose and suicide is a significant public health problem in the United States (U.S.). Approximately $20-30 \%$ of individuals with opioid use disorder have a history of both suicide attempt and unintentional opioid overdose. Objective: The objective of this study is to evaluate the feasibility of a standardized screen for suicide and overdose among patients receiving addiction treatment. Design: A cross-sectional study was conducted using a convenience sample of patients $(\mathrm{n}=113)$ recruited from two inpatient treatment programs. Methods: A modified version of the Patient Safety Screener (mPSS) was used to screen for suicidal ideation, suicide attempt, and overdose. The screen was administered in-person during treatment, and results were linked to administrative clinical data. Subjects $(n=108)$ and members of their clinical care team $(n=20)$ completed a screening acceptability survey. A positive mPSS was recorded if a patient reported suicidal ideation in the past two weeks, a suicide attempt, and/or an overdose in the past six months. Results: Fifty-eight percent of subjects had a positive mPSS screen, and 30.3\% reported suicidal ideation, suicide attempt, and overdose. Subjects and clinical staff reported that it was acceptable to screen for suicide attempt(s) and overdose(s). About a third of the clinical staff reported concerns about administration time $(\mathrm{n}=7)$ and impact on workflow $(\mathrm{n}=6)$. Conclusions: Both suicide and overdose are important patient safety factors that should be incorporated into treatment and discharge planning. The findings of this study support simultaneously screening 
for suicide and overdose in OUD inpatient treatment settings. Future research needs to determine whether screening improves provision of services and reduces self-injurious behavior. 


\subsection{Introduction}

Premature mortality associated with opioid-related overdose and suicide are significant public health problems in the United States (U.S.) (Bohnert and Ilgen, 2019; Cunningham et al., 2018; Oquendo and Volkow, Rockett et al., 2016; 2018; VanHouten et al., 2019). In 2018, there were 46,802 opioid-related overdoses $(14.3$ per 100,000) and 48,344 (14.2 per 100,000) suicides reported in the U.S. (Wilson, 2020; Xu et al., 2020). Previous research has estimated that 20 $30 \%$ of individuals with opioid use disorder (OUD) have reported a history of both a suicide attempt(s) and unintentional opioid overdose(s) (Darke et al., 2015; Maloney et al., 2010). People with OUD have elevated mortality rates, with suicide being one major contributor to premature mortality (Darke et al., 2011; Degenhardt et al., 2011; Darke et al., 2007; Wilcox et al., 2004). Additionally, overdose also plays a significant role in morbidity and mortality among individuals with OUD (Bohnert et al., 2010; Darke et al, 2015; Ravndal \& Vaglum, 1999). While previous reports have estimated that $25 \%$ of opioid overdose deaths are intentional (Oquendo \& Volkow, 2018), this is likely an underestimate given that suicide rates due to poisoning are underreported by approximately $30 \%$ (Donaldson et al., 2006). Furthermore, with the true proportion of non-fatal ODs identified as intentional being unknown, there exists a relationship between suicide and overdose that is difficult to ascertain.

Several studies have examined individuals with a history of both unintentional overdose(s) and suicide attempt(s) (Maloney et al., 2010; Rossow and Lauritzen, 1999). Subjects with this joint history manifest worse psychological and social functioning, as well as more severe drugrelated problems than those without a history of both unintentional overdose(s) and suicide attempt(s) (Bohnert et al., 2010). Ravndal and Vaglum found that $47 \%$ of clients in addiction treatment reported at least one suicide attempt, and $43 \%$ had at least one overdose-related 
hospitalization (1999). Patients with a history of suicide attempt(s) were more likely to report polydrug use, non-fatal overdose, and higher healthcare utilization (Ravndal \& Vaglum, 1999). The higher clinical severity observed among patients with a history of suicide and overdose reflects that these individuals need a broader range of services and intensive case management (Bohnert et al., 2010). Despite evidence of the overlap between overdose and attempted suicide, clinical screening and intervention have not been integrated.

Accurate identification of patients with a history or at risk of suicide and overdose is critical for treatment planning. In higher acuity treatment settings, there may be regulatory requirements to conduct universal suicide screening; however, there are no regulations to screen for a history of non-fatal overdose, and it is unknown whether overdose history is routinely assessed. The Joint Commission has recognized the importance of identifying suicide risk as part of routine clinical care and emphasizes the documentation of suicide assessments (both positive and negative findings), particularly in behavioral health care settings (The Joint Commission, 2016). However, suicide documentation in electronic medical records (EMRs) is often inconsistent or incomplete (Tanguturi et al., 2017). An estimated $83 \%$ of suicide decedents utilized healthcare in the year prior to their death, and $24 \%$ had a documented mental health diagnosis within 4 -weeks prior to death (Ahmedani et al., 2014). Missing or incomplete suicide documentation in the EMR may have led to missed opportunities to deliver prevention services. The mental health and addiction treatment systems are not integrated, and at a regulatory level, this may discourage simultaneous screening for overdose and suicide. Some behavioral health programs may be unable to treat patients with active suicidal ideation because their physical environment does not meet the requirements to ensure safety. Individuals with substance use disorders (SUDs) frequently have co-occurring mental health problems (Swendsen et al., 2010; Harris \& 
Barraclough, 1997), which can be very difficult to distinguish in the early phase of treatment, especially as withdrawal causes dysphoria. As many as $40 \%$ of patients seeking SUD treatment have a history of suicide attempt(s) (Roy \& Janal, 2007; Roy, 2009; Roy, 2010). Patients frequently present with depressive symptoms and significant functional impairments that may not only drive them to seek treatment, but also put them at higher risk for suicidal behavior (Yuodelis-Flores et al., 2015).

The feasibility of suicide screening has been demonstrated in different healthcare settings, including emergency departments (EDs) and primary care settings (Boudreaux et al., 2013; Boudreaux et al., 2016; King et al., 2009; Lish et al., 1996). Standardized suicide screening improves identification of at-risk individuals previously not identified (King et al., 2009; Boudreaux et al., 2016). The Emergency Department-Safety Assessment and Follow-up Evaluation (ED-SAFE), a quasi-experimental multisite clinical trial, found that standardized suicide screening resulted in a nearly two-fold increase in patient endorsement of any past or present intentional self-harm ideation or behavior (i.e. suicide risk detection) (Boudreaux et al., 2016). While presumably suicide screening is occurring in addiction treatment programs, there are no psychometrically validated overdose screening assessments, and it is unlikely that overdose history documentation specifies intentionality. Given the magnitude of the opioid and suicide epidemics (Rockett et al., 2019; Hedegaard et al., 2020), and due to evidence that prevention interventions can reduce the risk of suicide (Zalsman et al., 2016) and overdose (Irvine et al., 2019), integrated screening and risk evaluation are warranted. The objective of this study is to evaluate the feasibility of an integrated overdose and suicide standardized screen, in the context of inpatient treatment for individuals with OUD. This feasibility study includes assessment of screen acceptability for patients and clinical staff. 


\subsection{Methods}

$\underline{\text { Study Design }}$

A cross-sectional study was conducted, and patient data was collected using a structured in-person interview, a web-based survey (screening acceptability), and extraction of data from administrative clinical records. Clinician data was collected using a web-based survey on screen acceptability. Both participating patients and clinical staff provided written informed consent.

\section{$\underline{\text { Study Setting }}$}

Patients with an OUD were recruited from two treatment programs associated with a university-based health care system. One site was an acute inpatient dual-diagnosis unit that treats individuals who: 1) need medically managed detoxification and 2) are a danger to themselves or others (i.e. suicidal ideation/suicide attempt and/or homicidal ideation or actions). The second site was an inpatient treatment program that offered detoxification services and a 28day residential program; subjects were only recruited from the residential unit.

\section{$\underline{\text { Data Collection }}$}

\section{Study Sample.}

A convenience sample of patients were recruited from the acute inpatient unit starting in February 2019, and recruitment at the residential unit began in August 2019. Recruitment concluded at both sites in October 2019. Patients were included in the study if they were currently being treated at a recruitment site, had a confirmed diagnosis of OUD (communicated the attending physician or electronic medical record (EMR) documentation of OUD), and were willing to provide written informed consent. Patients were excluded if they were: 1) unable to read and write in English, 2) deemed not psychiatrically stable (i.e. no medical decision making capacity, active hallucinations, or homicidal ideation as determined by the attending physician), 
3) met the definition of incarceration (i.e. known to have a current warrant for their arrest, pending criminal charges, currently wearing an electronic ankle monitoring device, and/or mandated to treatment by law enforcement), 4) under the age of 18 years, or 5) were pregnant. Participation was completely voluntary, and compensation was not provided for study participation.

Clinical staff, who were actively employed during the study period, were recruited from the two sites. Staff recruitment occurred from December 2019 through February 2020. Staff were eligible for study participation if they: 1) provided treatment (i.e. conducted therapy groups, distributed medications, conducted therapeutic interventions, or were a treatment provider) to patients enrolled in the study, 2) attended treatment team meetings, and 3) were willing and able to provide written informed consent. Administrative staff without patient contact were ineligible to participate.

\section{Measures}

Administrative Clinical Database.

Administrative clinical data was extracted from a patient reported outcomes monitoring system used at both recruitment sites, details of which are provided elsewhere (Winstanley et al. 2020). Variables extracted included sex, race (white/non-white), ethnicity, education (less than high school, high school graduate/GED, or some college+), employment (employed full/parttime or unemployed), number of times arrested (lifetime), substance use history, and the adverse childhood experiences (ACEs) instrument. Substance use history included lifetime use (yes/no), age of first use, and route of administration for tobacco, alcohol, cocaine, marijuana, heroin, prescription opioids (illicit use), buprenorphine (illicit use), methamphetamines, and benzodiazepines. Other clinical characteristics extracted included lifetime injection drug use 
(yes/no), number of overdoses (lifetime), and age of first overdose. The 17 ACE items were categorized as mental health problems ( 2 items); alcohol or drug use ( 2 items); emotional, physical or sexual abuse ( 6 items); neglect (3 items); physical or emotional abuse by a significant other (1 item), and family dissolution (3 items). These categories were summed to generate a total score.

\section{Modified Patient Safety Screener.}

Subjects were administered a modified version of the Patient Safety Screener (PSS), developed by Boudreaux and colleagues $(2013,2015)$. A positive PSS is defined as suicidal ideation in the past two weeks or a suicide attempt in the past six months. The modified PSS (mPSS) included a sixth (new) question on previous drug overdose. The first question assesses depressed mood, and this item is not included in screen scoring. The second question was "over the past two-weeks, have you had thoughts of killing yourself?" and the response options are 1) yes, 2) no, 3) patient unable to complete, or 4) patient refused. The third question asked, "In your lifetime, have you ever attempted to kill yourself?" Subject responses included: 1) yes, 2) no, 3) patient unable to complete, or 4) patient refused. If the subject responded in the affirmative, a follow-up question asked, "when did this happen?" There were six possible responses: 1) within the past 24 hours (including today), 2) within the past month (not including today), 3) between one and six months ago, 4) more than six months ago, 5) patient was unable to complete, or 6) patient refused. The overdose question, added for this study, was modeled to match the suicide question just described. However, the words "attempted to kill yourself" were replaced with "overdose." The mPSS took approximately five minutes to administer, and a positive mPSS was defined as suicidal ideation in the past two weeks, a suicide attempt in the past six months, and/or overdose in the past six months. The 'in the past 24-hours and 'past month' response 
options were combined because subjects were not interviewed within the first 24 hours of their admission. For a subject with a positive mPSS screen, the attending physician and the subject's primary therapist were notified of the result via encrypted e-mail with a PDF of the subject's mPSS attached. If subject told a study team member that they were an immediate danger to themselves or others, the unit lead (i.e. charge nurse or lead therapist) was notified immediately and the attending physician was paged.

Patient Acceptability Survey.

Subjects who completed the mPSS were asked to complete a web-based patient acceptability survey. Subjects were asked to rate the extent to which they agreed or disagreed with six statements about comprehension, time burden, relevance of the screen to treatment, invasiveness of the screening questions, willingness to complete the screen questions in the future, and usefulness of the screening. The responses used a five-point Likert scale ranging from strongly disagree to strongly agree. Due to the distribution of responses, they were recoded as: 1) disagree, 2) neither disagree nor agree, or 3) agree. The survey took approximately ten minutes to complete.

\section{Staff Acceptability Survey.}

The staff acceptability survey comprised ten closed-ended and five open-ended questions. Twenty-four staff were eligible to participate in the study. Four were unable to be contacted because they were no longer employed at either site and their contact information was inaccessible. A web-based survey was sent via e-mail to 20 staff who had provided written informed consent. Staff who were unresponsive to the email request to complete the online survey, were provided a hard copy of the survey. The staff survey comprised questions on respondent age, job title, number of years working in current position, and number of years 
working in addiction treatment. Staff responded to four statements using a five-point Likert scale ranging from strongly disagree to strongly agree: 1) information provided by the safety screen is useful in clinical practice, 2) information provided on the safety screen changed how I treated the patient, 3) the safety screen placed an unnecessary burden on the clinical staff, and 4) the safety screen placed an unnecessary burden on the patient. Responses were recoded as agree, neither agree nor disagree, or disagree. The survey included a question on which patients should receive the mPSS, also referred to as the "safety screen" (all patients/high risk patients only/done at physician discretion/none). The open-ended questions included whether the screening information changed how patients were treated, improvements that would make the screen more useful, aspects of the screen that were most useful, potential barriers to implementing the screen into routine clinical practice and whether the screen should be integrated into clinical practice. $\underline{\text { Statistical Analysis }}$

Descriptive statistics were used to summarize the sociodemographic and clinical characteristics of the sample, as well as the acceptability surveys. Medians were reported for variables that were not normally distributed, with the exception of age. Statistics were reported for the overall sample, as well as by recruitment site to determine whether important clinical differences were associated with recruitment site.-Recruitment site differences were determined using Pearson's chi-square tests for categorical variables, t-test for means, and the MannWhitney U (Wilcoxon Ranksum) test to compare medians. Quantitative data analysis was conducted using Stata/SE 15.0 analytical software (StataCorp, 2017). Statistical significance was defined as $\mathrm{p} \leq 0.05$.

The open-ended questions in the staff acceptability survey were analyzed using a thematic analysis approach (Biggerstaff \& Thompson, 2008; Vaismoradi et al., 2013; Wagstaff 
\& Williams, 2014). The open-ended responses were coded and then combined into two overarching themes of advantages and disadvantages of the screen. These themes were used to subcategorize responses from clinical staff and were based on procedures outlined for previous qualitative methods (Vaismoradi et al., 2013). The qualitative analysis was hand coded by two members of the study team (ANS and ELW), and the study protocol was approved by the West Virginia University Institutional Review Board (IRB).

\subsection{Results}

Two hundred and eight patients were pre-screened for study eligibility. Ninety-five patients were excluded, and 113 provided written informed consent. The primary reasons for exclusion included inability to provide informed consent for mental health reasons $(29.5 \%, \mathrm{n}=28)$, patient discharge or leaving treatment before screening $(25.3 \%, n=24)$, pregnancy among female clients $(15.9 \%, n=15)$, declining to participate $(13.7 \%, n=13)$, legal issues/current arrest warrant $(8.4 \%$, $\mathrm{n}=8)$, failure to comply with study procedures $(4.2 \%, \mathrm{n}=4)$, and complications due to detoxification $(3.2 \%, n=3)$. Seventy-two patients $(63.7 \%)$ were recruited from the acute inpatient unit and $41(36.3 \%)$ were recruited from the residential treatment facility (Figure 3.1).

The overall sample was predominantly non-Hispanic (95.6\%), white $(95.6 \%)$ and male (64.3\%). The majority of the sample had at least a high school diploma or GED (46.9\%), and $23.9 \%$ of participants were employed in the past 30 days (Table 3.1). Clinical characteristics of the sample are reported in Table 3.1. There were no statistically significant differences in demographic or clinical characteristics by recruitment site, except subjects at the acute inpatient program were, on average, 3.3 years older than those recruited from the residential program (Table 3.1). 
Overall, $58.4 \%$ of the subjects screened positive on the mPSS, $38.1 \%$ had suicidal ideation in the past two weeks, $46.3 \%$ had a suicide attempt(s) in the past six months and $39.7 \%$ had overdosed in the past six months. Subjects recruited from the acute inpatient program were significantly more likely to report suicidal ideation in the past two weeks $(50.0 \%$ versus $17.1 \%$; $\mathrm{p}<0.01)$ and a suicide attempt in the past six months $(29.7 \%$ vs $5.9 \%$ or $11.8 \%, \mathrm{p}=0.02)$ than subjects recruited from the residential program.

The patient acceptability survey had a $95.6 \%$ response rate $(n=108)$. Overall, $18.5 \%$ of respondents found the screen time consuming. Additionally, $4.6 \%$ found it difficult to understand and $6.4 \%$ would not want to complete another screening. Furthermore, $5.6 \%$ were uncomfortable with the questions on the mPSS. Finally, $4.6 \%$ of subjects did not find the mPSS useful and $6.5 \%$ did not understand how the screening related to their treatment. Across all of the items in acceptability survey, $14-23 \%$ endorsed 'neither agree nor disagree.' Screen acceptability did not vary by recruitment site (see Table 3.3).

Twenty staff completed the acceptability survey, with a response rate of $83.3 \%$. The mean age of staff respondents was 35.0 years $(\mathrm{SD}=10.1)$. A quarter of the staff was physicians, another quarter drug and alcohol technicians, $20.0 \%$ were clinical therapists or counselors, $20.0 \%$ were social workers, $5.0 \%$ were nurses, and $5.0 \%$ were clinical coordinators. Ninety-five percent of staff agreed the mPSS provided useful information, $45.0 \%$ found it provided information that changed how they treated the patient, $70.0 \%$ reported it did not place an unnecessary burden on the patients, $85.0 \%$ thought all patients should receive the mPSS, $100 \%$ of staff thought the assessment was useful in clinical practice, and $95.0 \%$ thought the mPSS should be integrated into standard clinical practice. 
Open-ended items in the staff acceptability survey were categorized as follows: 1) treatment change, 2) improvements to increase usefulness in clinical practice, 3) aspects of the safety screen found most useful, 4) extant barriers to clinical implementation, and 5) integration into clinical practice. Overall analysis of the open-ended response data found that respondents reported 12 advantages and 9 disadvantages. Most respondents found the results of the mPSS to be informative $(n=17)$ and stated that "It allows for clinicians to make more educated, evaluated decisions," and "I think it is a good tool to measure where the patient is mentally when they arrive to the facility."

Other advantages of the mPSS included the ability to convey risk $(n=8)$, assess the patient $(n=7)$, evaluate safety $(n=7)$, the completeness of the assessment $(n=5)$, its usefulness $(n=4)$ in clinical practice, and informing patient history ( $n=3$; Figure 3.2). For instance, the clinical therapists and social workers conveyed:

"This helps better understand this person's past coping mechanisms."

"The safety of the guest is the utmost importance. This is another tool to ensure that the most is being done to keep them safe."

"It provides more detail about the patients history so we can tailor treatment to the individual and reduce risk."

Other advantages mentioned by respondents included reporting comorbidities $(n=2)$, clinical management $(n=1)$, efficiency $(n=1)$, individual care $(n=1)$, and relatability $(n=1)$.

The most frequently reported disadvantage of the mPSS was time to complete $(n=7)$. This problem was compounded by other issues, such as workflow $(n=6)$, communication $(n=4)$, patient resistance $(n=2)$, and concerns about patient re-traumatization $(n=2)$. For example: "While some of these questions may be asked/answered in the admissions process, it would be 
nice to have them definitely in their chart and always in one spot." While, other staff members provided the following feedback: "Takes a long time to administer, was sometimes triggering to the patients." A complete list of themes is depicted in Figure 3.2.

\subsection{Discussion}

This study found that nearly $60 \%$ of subjects receiving inpatient OUD treatment had suicidal ideation, suicide attempts and/or overdose. A fifth of the subjects had a history of only overdose(s), whereas a third had current suicidal ideation and a history of overdose(s) and suicide attempt(s). Lifetime suicide attempt(s) (46.3\%) in this study was consistent with existing research (Roy, 2010; Roy, 2002; Ravndal \& Vaglum 1999); however, the prevalence of a lifetime overdose $(60.2 \%)$ was still higher than reported in previous research of patients seeking addiction treatment (Ross et al., 2005). This difference speaks to the point that, while suicide history is important to address in addiction treatment, screening for overdose plays an equally important role in understanding patient history as a whole.

The most positive feedback received was for comprehension of screening questions. Given that more than half of subjects had a high school education or less, it is important that $83.3 \%$ of subjects reported that the mPSS questions were easy to understand. Furthermore, although patients in both clinical settings were required to be psychiatrically cleared by the attending physician, some patients were receiving various pharmaceutical interventions to treat their addiction or psychiatric conditions. Particularly for patients in the acute inpatient settings, some patients may have experienced the effects of detoxification and possibly were still adjusting to their new environment (Rapeli et al., 2006). Thus, it is essential to have a screening tool with easily comprehended questions. A minority of respondents (18.5\%) reported that the screen was 
time consuming; while the survey question was in reference to administration time for the mPSS only, subjects might interpret it as the time to complete the entire research interview.

Despite clinical concerns that patients may be uncomfortable completing a standardized screen on suicide and overdose, $94.4 \%$ of patients were comfortable with the screen. A previous literature review found most studies reported that only a small minority of patients, who participated in trauma-focused research, experienced distress (Legerski \& Bunnell, 2010). Moreover, most studies reported that negative feelings dissipate quickly, and that the great preponderance of patients participating in research feel rewarded (Legerski \& Bunnell, 2010). A study by Biddle and colleagues measured the emotional state of adult and adolescent participants, with a history of self-harm or suicidality, before and after interviews on their personal experiences (Biddle et al., 2013). They reported that over 50\% of participants found talking about their experiences beneficial. Affirming previous findings, $73.2 \%$ of patients in our study reported the mPSS was useful and $78.7 \%$ understood how the questions pertained to their current treatment. Nevertheless, it is important to consider that the screening questions on potentially traumatic events may cause patient distress (LeFevre, 2014). In these instances, it is important to address patient concerns, provide them with the appropriate resources, and allow them time to process their emotions before ending the interview (Espisto, 2019; LeFevre, 2014)

All staff reported that the mPSS was useful in clinical practice and was a minimal burden to patients. All but one agreed that the instrument should be integrated into standard clinical procedures. However, the clinical staff did not administer the screen, and it was not administered upon treatment entry. If clinicians had to administer the screen, overall acceptability may have been lower. Conversely, if the screen was administered at intake and integrated into the standard workflow, then it may have had higher clinical utility. There was disagreement on whether the 
screening results changed how the patient was treated, and this variation may be partially explained by the respondent's clinical role and involvement in treatment planning.

The staff acceptability survey also included six open-ended response questions. Several disadvantages were frequently reported by the staff. Seven reported administration time as a disadvantage. This involvement may actually reflect disruption to clinical workflow and indicate a barrier in terms of the division of labor regarding which staff members would be tasked with administering the mPSS in the future. Furthermore, workflow could be impacted by several features of the screen, such as when the mPSS is administered and how the results are communicated. For the current study, mPSS was administered during intake or the patient's unstructured free time; however, the results were often not communicated to the therapist prior to interview. Although results were provided to the clinical staff, results were not recorded in the EMR or communicated at the same point in treatment. This knowledge gap resulted in potential duplication of work and redundancy. It bolsters the case for having the mPSS incorporated directly into the patient's EMR and readily accessible for care team review. Conversely, another common theme was that staff treat all patients uniformly irrespective of background. This could partially be due to the large number of staff members completing the survey, who did not have one-on-one patient contact, but rather interacted with patients in group settings. One such group was drug and alcohol technicians, who comprised $25.0 \%$ of survey respondents. Regarding barriers to implementation, time was the biggest disadvantage reported by staff members. Overall, the mPSS was thought to be efficient in providing a concise reporting tool for conveying safety risk. However, patients who screened positive would ultimately require longer assessments. This means a longer time commitment for the patient and for the staff member administering the detailed suicidal ideation, suicide attempt, and/or overdose assessment- 
depending on the initial mPSS result. However, despite these barriers and concerns, $95.0 \%$ of staff reported the mPSS should be integrated into standard clinical practice.

\section{$\underline{\text { Limitations }}$}

As the study sample was recruited from an inpatient population, the results may not generalize to other treatment modalities. Likewise, the majority of the subjects recruited for this study was predominantly white and non-Hispanic, and results might not generalize to more diverse patient populations. In order to integrate this instrument into clinical practice, members of the clinical team would need to administer the screening to patients or have the screen selfadministered by patients. Integration may require more time from the staff to debrief with patients that become distressed. Although it is ideal to administer the mPSS at intake to help inform treatment planning, the accuracy of the results may be compromised if the patient arrives intoxicated or has not established rapport with the treatment team.

\section{$\underline{\text { Future Directions }}$}

Overall, additional research is needed to determine the optimal timing of mPSS administration and to establish the test-retest validity of the instrument. Furthermore, additional research needs to be conducted to establish the prospective validity of the tool and if the tool changes clinical practice and/or the provision of prevention services. Stricter criteria for when to administer the mPSS should be established to obtain consistent results, and/or criteria set for rescreening at various points throughout the inpatient stay to address fluctuations in suicidality. The next step would be to test the mPSS among patients enrolled in outpatient treatment programs, so as to assess overdose and suicide in patients at varying stages of recovery. 


\subsection{Conclusions}

Based on the current findings from this study, concurrent screening for overdose and screening is acceptable and warranted. Through a dual screening procedure, this may increase the clinical awareness of the overlap between overdose and suicide among patients with OUD. For this reason, suicidal behaviors warrant more clinical and research attention in the addiction field (Yuodelis-Flores et al., 2015), and support the need to for all clients receiving addiction treatment services to also be screened and monitored for suicidality and overdose. This finding indicates screening for both suicide and overdose is an important clinical contributor to overall patient safety assessment and provides crucial information for treatment and discharge planning. Both patients and staff found the mPSS informative and appropriate for assessment and approved of the application of instrument to the clinical setting. In general, the greater the burden of risk factors, the higher the likelihood for suicide attempt or death by suicide. The information gathered in this study shows that the mPSS is an appropriate screening tool to address these issues. Additional research is necessary to determine whether screening improves provision of services and reduces self-injurious behavior.

\subsection{Acknowledgements}

Funding Source: ANS received funding from the National Institute of General Medical Sciences of the National Institutes of Health (1 T32 GM132494). 


\begin{tabular}{|c|c|c|c|c|}
\hline Characteristics & $\begin{array}{c}\text { Overall Sample } \\
(\mathrm{n}=113)\end{array}$ & $\begin{array}{c}\text { Acute Inpatient } \\
(\mathrm{n}=72)\end{array}$ & $\begin{array}{c}\text { Residential Program } \\
(\mathrm{n}=41)\end{array}$ & p-value \\
\hline Mean Age (years), $(\mathrm{SD})^{*}$ & $35.2(9.2)$ & $36.4(9.0)$ & $33.1(9.3)$ & 0.02 \\
\hline Sex (\% Male) & $72(63.7)$ & $47(65.3)$ & $25(61.0)$ & 0.65 \\
\hline White (\%) & $108(95.6)$ & $68(94.4)$ & $40(97.6)$ & 0.65 \\
\hline Non-Hispanic (\%) & $108(95.6)$ & $68(94.4)$ & $40(97.6)$ & 0.65 \\
\hline Employed (\%) & $27(23.9)$ & $21(29.2)$ & $6(14.6)$ & 0.08 \\
\hline Education $(\%)$ & & & & 0.42 \\
\hline Less than High School & $18(15.9)$ & $9(12.5)$ & $9(22.0)$ & \\
\hline High School Grad/GED & $53(46.9)$ & $35(48.6)$ & $18(43.9)$ & \\
\hline Some College/College Grad & $42(37.2)$ & $28(38.9)$ & $14(34.1)$ & \\
\hline Lifetime Arrests (Median) & 5.0 & 5.0 & 4.0 & 0.23 \\
\hline \multicolumn{5}{|l|}{ Ever Used, Lifetime (\% Yes) } \\
\hline Tobacco & $112(99.1)$ & $71(98.6)$ & $41(100.0)$ & 1.00 \\
\hline Alcohol & $112(99.1)$ & $71(98.6)$ & $41(100.0)$ & 1.00 \\
\hline Cocaine & $102(90.3)$ & $63(87.5)$ & $39(95.1)$ & 0.32 \\
\hline Marijuana & $111(98.2)$ & $70(97.2)$ & $41(100.0)$ & 0.53 \\
\hline Heroin & $100(88.5)$ & $62(86.1)$ & $38(92.7)$ & 0.37 \\
\hline Prescription Opioids & $104(92.0)$ & $68(94.4)$ & $36(87.8)$ & 0.28 \\
\hline Illicit/Non-Medical Buprenorphine Use & $49(43.4)$ & $29(40.3)$ & $20(48.8)$ & 0.38 \\
\hline Methamphetamines/Amphetamines & $98(86.7)$ & $61(84.7)$ & $37(90.2)$ & 0.57 \\
\hline Benzodiazepines & $74(65.5)$ & $61(84.7)$ & $37(90.2)$ & 0.64 \\
\hline \multicolumn{5}{|l|}{ Mean age of First Use (Years), (SD) } \\
\hline Tobacco* & $13.1(5.0)$ & $12.9(5.2)$ & $13.6(4.7)$ & 0.62 \\
\hline Alcohol $^{*}$ & $13.8(6.9)$ & $13.9(8.4)$ & $13.7(3.1)$ & 0.71 \\
\hline Cocaine $^{*}$ & $19.0(4.9)$ & $18.8(5.0)$ & $19.4(4.7)$ & 0.69 \\
\hline Marijuana $^{*}$ & $14.3(3.9)$ & $14.7(4.5)$ & $13.6(2.4)$ & 0.18 \\
\hline Heroin* & $24.8(9.3)$ & $24.4(9.1)$ & $25.4(9.6)$ & 0.61 \\
\hline Prescription Opioids ${ }^{*}$ & $18.3(6.4)$ & $18.6(7.0)$ & $17.6(5.3)$ & 0.56 \\
\hline Illicit/Non-Medical Buprenorphine Use $^{*}$ & $25.6(9.1)$ & $25.9(10.2)$ & $25.2(7.5)$ & 0.71 \\
\hline Methamphetamines/Amphetamines $^{*}$ & $27.4(8.6)$ & $27.5(8.3)$ & $27.3(9.2)$ & 0.71 \\
\hline Benzodiazepines $^{*}$ & $20.4(8.2)$ & $21.4(8.6)$ & $18.9(7.3)$ & 0.27 \\
\hline Ever Inject Drugs (\%Yes, Lifetime) & $88(77.9)$ & $58(80.6)$ & $30(73.2)$ & 0.36 \\
\hline Mean Age First Injection Drug Use (SD) ${ }^{*}$ & $25.1(8.4)$ & $25.6(8.9)$ & $24.1(7.4)$ & 0.68 \\
\hline Median Number of Lifetime Overdoses & 1.0 & 1.0 & 1.0 & - \\
\hline $\begin{array}{l}\text { Median Number of Overdoses (Past Six- } \\
\text { Months) }\end{array}$ & 3.0 & 3.0 & 2.5 & 0.64 \\
\hline Mean Age First Overdose (Years) (SD) ${ }^{*}$ & $26.5(10.3)$ & $27.1(9.9)$ & $25.4(11.2)$ & 0.33 \\
\hline ACE Total Domain Score & $4.8(1.8)$ & $4.8(1.7)$ & $4.8(1.9)$ & 0.91 \\
\hline \multicolumn{5}{|c|}{$\begin{array}{l}\text { Table 3.1: For ease of comparison ages were reported as means. However, variables designated with an "*” did not follow a normal } \\
\text { distribution therefore medians were compared using Wilcoxon rank-sum tests. Unless otherwise noted variables were compared using chi- } \\
\text { squared, Fisher's exact, or t-tests where appropriate. Additional details are described in the methods section. Statistical significance was set at } \\
p \leq 0.05 .\end{array}$} \\
\hline
\end{tabular}




\begin{tabular}{|c|c|c|c|c|c|c|c|}
\hline \multirow[t]{2}{*}{$\begin{array}{l}\text { 2A) Modified Patient Safety Screener (mPSS) } \\
\text { Items }\end{array}$} & \multicolumn{2}{|c|}{$\begin{array}{c}\text { Overall Sample } \\
(\mathrm{n}=113)\end{array}$} & \multicolumn{2}{|c|}{$\begin{array}{l}\text { Acute Inpatient } \\
\qquad(\mathrm{n}=72)\end{array}$} & \multicolumn{2}{|c|}{$\begin{array}{c}\text { Residential Program } \\
(\mathrm{n}=41)\end{array}$} & \multirow[t]{2}{*}{ p-Value } \\
\hline & n & $\%$ & $\mathbf{n}$ & $\%$ & $\mathbf{n}$ & $\%$ & \\
\hline Depression in past two-weeks (Yes) & 101 & 89.4 & 64 & 88.9 & 37 & 90.2 & 1.00 \\
\hline Suicidal Ideation in past-two weeks (Yes) & 43 & 38.1 & 36 & 50.0 & 7 & 17.1 & $<0.01$ \\
\hline Lifetime Suicide Attempt (Yes) & 54 & 47.8 & 37 & 51.4 & 17 & 41.5 & 0.31 \\
\hline Most Recent Suicide Attempt & & & & & & & 0.02 \\
\hline Within the last month & 12 & 22.2 & 11 & 29.7 & 1 & 5.9 & \\
\hline Between 1-6 months ago & 13 & 24.1 & 11 & 29.7 & 2 & 11.8 & \\
\hline More than 6 months ago & 29 & 53.7 & 15 & 40.6 & 14 & 82.4 & \\
\hline Ever Overdose (Yes) & 68 & 60.2 & 43 & 59.7 & 25 & 61.0 & 1.00 \\
\hline Most Recent Overdose & & & & & & & 0.15 \\
\hline Within the last month & 14.0 & 20.6 & 12 & 27.9 & 2 & 8.0 & \\
\hline Between 1-6 months ago & 13.0 & 19.1 & 8 & 18.6 & 5 & 20.0 & \\
\hline More than 6 months ago & 41.0 & 60.3 & 23 & 53.5 & 18 & 72.0 & \\
\hline 2B) Positive mPSS Response Combinations & \multicolumn{2}{|c|}{$\begin{array}{c}\text { Overall Sample } \\
(\mathrm{n}=66)\end{array}$} & \multicolumn{2}{|c|}{$\begin{array}{l}\text { Acute Inpatient } \\
(\mathrm{n}=52)\end{array}$} & \multicolumn{2}{|c|}{$\begin{array}{c}\text { Residential Program } \\
(\mathrm{n}=14)\end{array}$} & p-Value \\
\hline Suicidal Ideation Only & 5 & 7.58 & 5 & 9.62 & 0 & 0.0 & 0.57 \\
\hline Suicide Attempt Only & 4 & 6.1 & 3 & 5.8 & 1 & 7.1 & 1.00 \\
\hline Overdose Only & 13 & 19.7 & 8 & 15.4 & 6 & 35.7 & 0.09 \\
\hline Suicidal Ideation + Suicide Attempt & 11 & 16.7 & 10 & 19.2 & 1 & 7.1 & 0.43 \\
\hline Suicidal Ideation + Overdose & 7 & 10.6 & 6 & 11.5 & 1 & 7.1 & 1.00 \\
\hline Suicide Attempt + Overdose & 6 & 9.1 & 5 & 9.6 & 1 & 7.1 & 1.00 \\
\hline Suicidal Ideation + Suicide Attempt + Overdose & 20 & 30.3 & 15 & 28.9 & 5 & 35.7 & 0.62 \\
\hline
\end{tabular}




\begin{tabular}{|c|c|c|c|c|}
\hline Patient Acceptability Questions (Frequency), (\%) & $\begin{array}{l}\text { Overall Sample } \\
\quad(\mathrm{n}=108)^{\dagger}\end{array}$ & $\begin{array}{c}\text { Acute } \\
\text { Inpatient } \\
(\mathrm{n}=69)\end{array}$ & $\begin{array}{c}\text { Residential } \\
\text { Program } \\
(\mathrm{n}=39)\end{array}$ & p-Value \\
\hline The self-harm screen was time consuming. & & & & 0.99 \\
\hline Disagree & $63(58.3)$ & $40(58.0)$ & $23(59.0)$ & \\
\hline Neither disagree nor agree & $25(23.2)$ & $16(23.2)$ & $9(23.1)$ & \\
\hline Agree & $20(18.5)$ & $13(18.8)$ & $7(18.0)$ & \\
\hline The questions in the screening questionnaire were hard to understand. & & & & 0.78 \\
\hline Disagree & $90(83.3)$ & $56(81.2)$ & $34(87.2)$ & \\
\hline Neither disagree nor agree & $13(12.0)$ & $9(13.0)$ & $4(10.3)$ & \\
\hline Agree & $5(4.6)$ & $4(5.8)$ & $1(2.5)$ & \\
\hline In the future I would not want to complete another self-harm screen. & & & & 0.29 \\
\hline Disagree & $83(76.9)$ & $56(81.2)$ & $27(69.2)$ & \\
\hline Neither disagree nor agree & $18(16.7)$ & $9(13.0)$ & $9(23.1)$ & \\
\hline Agree & $7(6.4)$ & $4(5.8)$ & $3(7.7)$ & \\
\hline The questions on the self-harm screen made me uncomfortable. & & & & 0.35 \\
\hline Disagree & $85(78.7)$ & $51(73.9)$ & $34(87.2)$ & \\
\hline Neither disagree nor agree & $17(15.7)$ & $13(18.8)$ & $4(10.3)$ & \\
\hline Agree & $6(5.6)$ & $5(7.3)$ & $1(2.5)$ & \\
\hline The questions asked in the self-harm screen were not useful. & & & & 0.71 \\
\hline Disagree & $79(73.2)$ & $49(71.0)$ & $30(76.9)$ & \\
\hline Neither disagree nor agree & $24(22.2)$ & $17(24.6)$ & $7(18.0)$ & \\
\hline Agree & $5(4.6)$ & $3(4.4)$ & $2(5.1)$ & \\
\hline I do not understand how the self-harm screen relates to my treatment. & & & & 0.57 \\
\hline Disagree & $85(78.7)$ & $53(76.8)$ & $32(82.1)$ & \\
\hline Neither disagree nor agree & $16(14.8)$ & $10(14.5)$ & $6(15.4)$ & \\
\hline Agree & $7(6.5)$ & $6(8.7)$ & $1(2.5)$ & \\
\hline \multicolumn{5}{|c|}{$\begin{array}{l}\text { Table 3.3: Comparisons were made using chi-squared or Fisher's exact tests where appropriate. Statistical significance was set at } p \leq 0.05 \text {. Additiona } \\
\text { details are described in the methods section. } \\
{ }^{\dagger} \text { Five study participants did not complete the survey, two interviews were discontinued before the patient acceptability survey by the interviewer for } \\
\text { safety reasons and three patients could not complete the patients acceptability survey due to time constraints. }\end{array}$} \\
\hline
\end{tabular}




\begin{tabular}{|c|c|}
\hline \multicolumn{2}{|l|}{ 3.4A) Staff Characteristics $(n=20)$} \\
\hline Age (Mean (years)), (SD) & $35.0(10.1)$ \\
\hline \multicolumn{2}{|l|}{ Job Title $(\%),(n)$} \\
\hline Drug \& Alcohol Technician & $25.0(5.0)$ \\
\hline Physician & $25.0(5.0)$ \\
\hline Social Worker & $20.0(4.0)$ \\
\hline Clinical Therapist/Counselor & $20.0(4.0)$ \\
\hline Nurse & $5.0(1.0)$ \\
\hline Clinical Coordinator & $5.0(1.0)$ \\
\hline Years Worked in Current Position (mean), (SD) & $3.4(4.8)$ \\
\hline Total Years in AOD* Field (mean), (SD) & $9.2(8.0)$ \\
\hline \multicolumn{2}{|l|}{ 3.4B) Acceptability Questionnaire Responses (Frequency) (\%) } \\
\hline \multicolumn{2}{|l|}{ The information provided by the safety screen is useful to clinical practice. } \\
\hline Disagree & $1(5.0)$ \\
\hline Neither disagree nor agree & $0(0.0)$ \\
\hline Agree & $19(95.0)$ \\
\hline \multicolumn{2}{|l|}{ Information provided on the safety screen changed how I treated the patient. } \\
\hline Disagree & $3(15.0)$ \\
\hline Neither disagree nor agree & $8(40.0)$ \\
\hline Agree & $9(45.0)$ \\
\hline \multicolumn{2}{|l|}{ The safety screen places unnecessary burden on the clinical staff. } \\
\hline Disagree & $16(80.0)$ \\
\hline Neither disagree nor agree & $4(20.0)$ \\
\hline Agree & $0(0.0)$ \\
\hline \multicolumn{2}{|l|}{ The safety screen places unnecessary burden on the patient. } \\
\hline Disagree & $14(70.0)$ \\
\hline Neither disagree nor agree & $6(30.0)$ \\
\hline Agree & $0(0.0)$ \\
\hline \multicolumn{2}{|l|}{ Which patients should receive safety screening? } \\
\hline All patients & $17(85.0)$ \\
\hline High risk patients only & $1(5.0)$ \\
\hline Done at the discretion of the physician & $2(10.0)$ \\
\hline None & $0(0.0)$ \\
\hline Is the self-harm assessment useful for clinical practices (\% Yes) & $20(100.0)$ \\
\hline Should the patient safety screen become part of clinical practice? (\% Yes) & $19(95.0)$ \\
\hline \multicolumn{2}{|l|}{ 4C) Staff Acceptability Open-Ended Questions (Frequency) (\%) } \\
\hline Please explain how the information did or did not change how you treated the patient. & $10(50.0)$ \\
\hline What improvements could be made to the safety screen to make it more useful in clinical practice? & $7(35.0)$ \\
\hline What aspects of the safety screen did you find the most useful? & $13(65.0)$ \\
\hline What barriers might prevent the patient safety screen from being implemented into routine clinical practice? & $11(55.0)$ \\
\hline Should the patient safety screen become part of clinical practice? & $12(60.0)$ \\
\hline \multicolumn{2}{|c|}{$\begin{array}{l}\text { Table 4: Comparisons were not made between treatment facilities due to small sample size recruited for this portion of the study. } \\
\text { Open-ended question responses are reported in Figure 2. Additional details are provided in the methods section. } \\
\text { *AOD=Alcohol or Drug }\end{array}$} \\
\hline
\end{tabular}


Figure 3.1: Recruitment Diagram

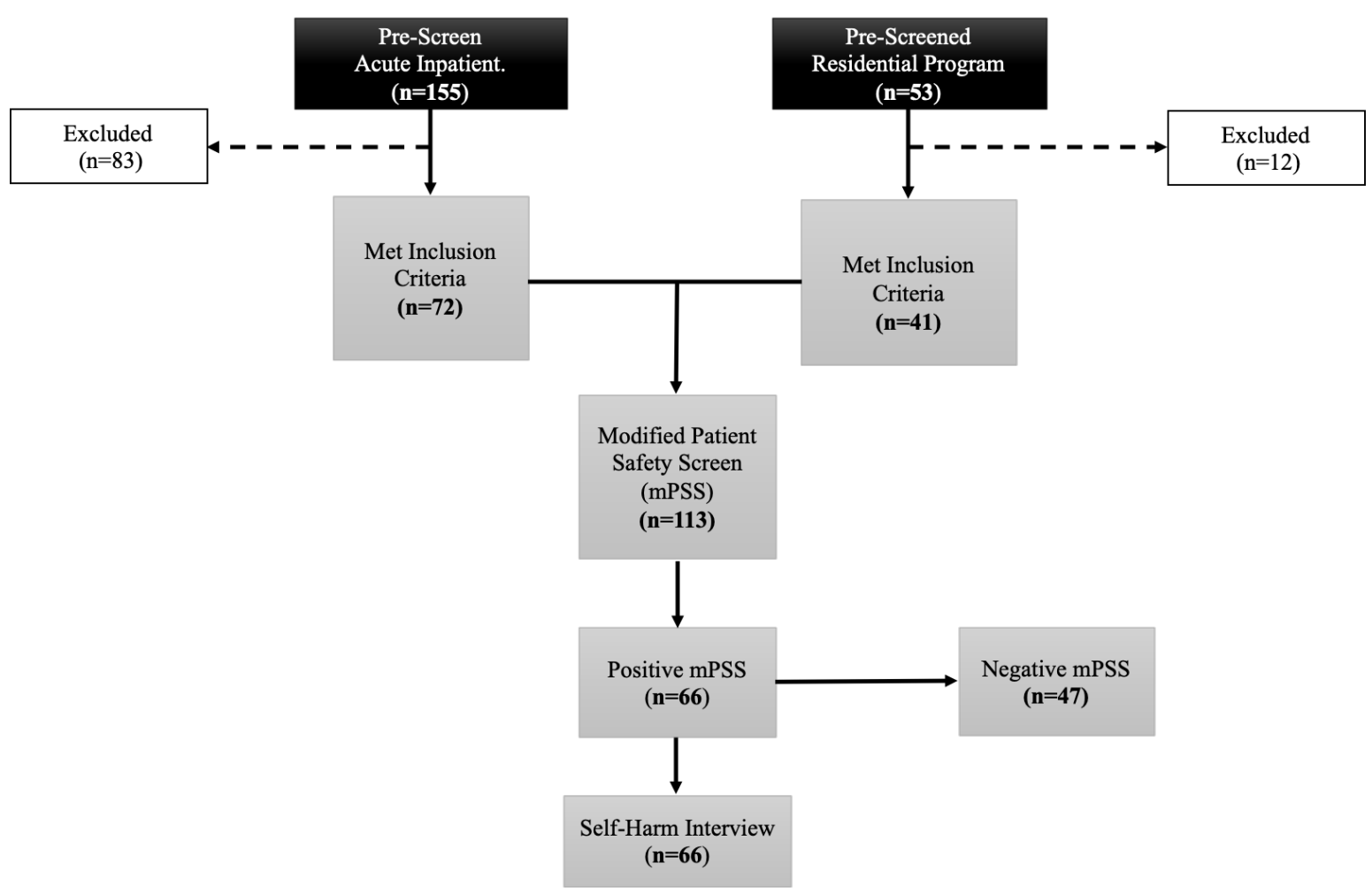


Figure 3.2: Open-ended Response Summary from Staff Acceptability Questionnaire

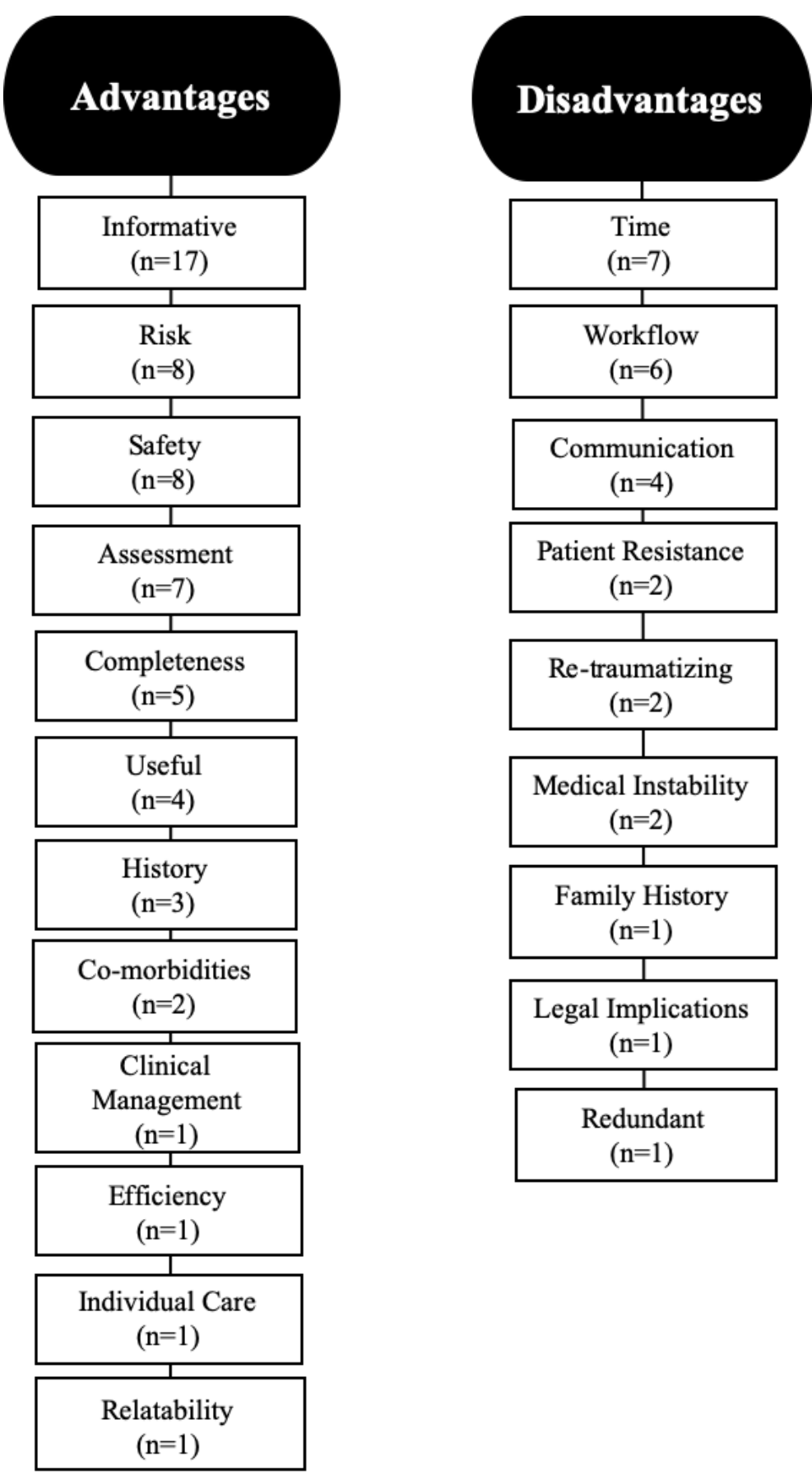




\section{Chapter 4}

\section{Distinguishing Clinical Factors Associated with Unintentional Overdose, Suicidal Ideation, Suicide Attempt, and Opioid Use Disorder}

\subsection{Abstract}

Background: Both suicide and opioid use disorder (OUD) represent significant threats to public health. Premature mortality due to opioid-related overdose and suicide continues to rise in the United States. Objective: The objective of this study is to examine the clinical factors that may distinguish three patient groups: 1) unintentional overdose(s) (OD), 2) suicidal ideation or suicide attempt(s) (SI/SA), and 3) suicidal ideation or suicide attempt and unintentional overdose (SI/SA/OD). Design: This was a cross-sectional study conducted in a subset of subjects recruited from two inpatient addiction treatment facilities $(n=66)$. Methods: Subjects were categorized as having a history of unintentional OD $(n=13)$; SI/SA $(n=33)$; or SI/SA/OD ( $n=20)$. Multinomial logistic regression models were used to determine whether adverse childhood experiences, self-injurious behaviors, substance use history, overdose history, and past year stressful life events were differentially associated with history of SI/SA/OD. Results: Overall, $19.7 \%$ of the study sample reported an overdose, while $50.0 \%$ reported SI/SA, and $30.3 \%$ reported SI/SA/OD. Subjects in the overdose group were more likely to have used heroin in the 24-hours preceding their most recent overdose compared to either the SI/SA or SI/SA/OD groups. In the multivariable model, subjects with history of SI/SA had higher adverse childhood experience scores and more subjects with history of SI/SA endorsed childhood physical abuse and teen dating violence. Conclusions: Overall, history of overdose is distinct from suicidal ideation and attempt. However, patients with SI/SA/OD appear to have greater clinical severity. More thorough evaluation of drugs involved in overdose and history of self-injury may help distinguish future risk and better inform treatment planning. More thorough evaluation of drugs 
involved in overdose and history of self-injury may help distinguish future risk and better inform treatment planning.

\subsection{Introduction}

Premature mortality due to opioid-related overdose and suicide represent a significant threat to public health in the United States (U.S.) across all age groups; both of which are leading causes of injury death that have continued to rise over the past decade (Connery et al., 2019; Bohnert \& Ilgen, 2019; Oquendo and Volkow, 2018). However, since drug overdose is the third most frequently used method of suicide the overlap between unintentional overdose and suicide is often murky (Centers for Disease Control and Prevention, 2017). Large population studies confirm that individuals with OUD, who survive an opioid-related overdose, remain at significantly elevated risk for repeat, fatal overdose (Olfson et al., 2018b) and death by suicide (Bogdanowicz et al., 2016; Olfson et al., 2018a). Multiple clinical factors are associated with opioid-related overdose and/or suicidal behavior among individuals with OUD, including use of heroin (Dai et al., 2019; Darke et al., 2015; Hakansson et al., 2008; Park et al., 2018), history of injection drug use (IDU) (Bohnert et al., 2011; Hakansson et al., 2008), polysubstance use (Bohnert et al., 2011; Bradvik et al., 2009; Darke et al., 2004; Jonsson et al., 2007), benzodiazepine use (Bohnert et al., 2017; Darke, 1996; Hakansson et al., 2008; Jones and McAninch, 2015), co-occurring psychiatric disorder (Bohnert et al., 2017; Darke et al., 2004, 2015; Maloney et al., 2007), history of sexual/physical trauma (Bohnert et al., 2011; Darke et al., 2004; Stein et al., 2017), violence directed toward others (Bohnert et al., 2011; Hakansson et al., 2008), and history of suicide attempt or persistent suicidal ideation (Darke et al., 2004, 2007;

Hakansson et al., 2008; Maloney et al., 2007; Pan et al., 2014). 
A 2010 review by Bohnert and colleagues reported that overdose and suicide are distinct, yet potentially related events. This review cited that the primary association between overdose and suicidal behaviors was based upon a single study by Darke and Ross in 2001, which found that individuals who had overdosed were also more likely to have attempted suicide, compared to those who had never overdosed. However, this association was largely explained by overdose as a method of suicide attempt, and when intentional overdose cases were removed from the analysis, it was no longer statistically significant (Darke \& Ross, 2001). Furthermore, when examining individuals with non-fatal overdoses and suicide attempts separately, both groups had worse psychological and social functioning as well as more severe drug-related problems (Bohnert et al., 2010). Patients with a history of both overdose and suicide attempt have significantly impaired functioning and increased severity in substance use, which necessitates delivery of higher intensity services compared to their peers without either previous suicide attempt(s) or overdose (Bohnert et al., 2010). Individuals with OUD who attempt suicide by drug overdose are more likely to use prescription medications, cocaine, alcohol, and sedatives; whereas, heroin is more likely to be used in unintentional overdoses (Bohnert et al., 2010; Pfab et al., 2006; Ravndal and Vaglum, 1999; Schifano et al., 2006). Existing research has not adequately accounted for precursory events and behaviors, such as trauma and self-injury, which may contribute to the intersection between overdose and suicide. Poor social functioning, psychiatric decline, and higher severity of substance use are overlapping risk factors for overdose and suicide that require further investigation.

Nonfatal overdoses are associated with increased acute care utilization and elevated risk of subsequent mortality. Between 2005 and 2014, opioid-related emergency department visits in the U.S. nearly doubled, while hospital admissions related to opioid use increased by nearly two- 
thirds (Weiss et al., 2016). This has resulted in increased clinical interest in characterizing the subsequent risk of overdose and non-overdose related causes of death (Olfson et al., 2018). Previous research has shown that OUD is associated with an elevated risk of premature mortality (Pierce et al., 2015). In the year after a nonfatal opioid overdose, patients died at approximately 24 times the rate of the general population (Olfson et al., 2018). Beyond stabilizing patients after nonfatal opioid overdoses, clinicians in acute care settings have opportunities to initiate medications for treatment of opioid use disorder and engage patients in ongoing treatment, harm reduction, or specialty addiction services (D’Onofrio et al., 2017). After a nonfatal overdose, men, but especially women, were at exceptionally high risk of suicide. In a recent study of veterans, the association between OUD and suicide was significantly stronger among women (Bohnert et al., 2017). The rate of suicide during the first-year post overdose, for women (29.3 per 10000 person-years), resembled the rate of suicide following the year after SIB (30.9 per 10 000 person-years) (Olfson et al., 2017). Although SIB and nonfatal overdose are distinct behaviors that utilize different prevention strategies, they can co-occur within an individual, and careful clinical assessment may be necessary to delineate them. A high risk of suicide after nonfatal opioid overdose underscores the importance of careful mental health assessment with appropriate follow-up for selected patients (Oqueno et al., 2017). Understanding these risks could inform efforts to provide preventive and potentially lifesaving medical care after a nonfatal opioid overdose and better integrate mental health care with the treatment of OUDs.

The objective of this study is to examine the clinical profiles of three patient groups: 1) unintentional overdose (OD), 2) suicidal ideation and suicide attempt (SI/SA), and 3) suicidal ideation, suicide attempt, and unintentional overdose (SI/SA/OD). These objectives were accomplished by examining the age of onset of substance use, age of initiation of SIB, last 
episode of self-injury, ACE domain scores, and past year stressful life experiences for each of the three groups. Previous research has found an association between higher ACE scores and younger age of onset of opioid use, recent injection drug use, and lifetime overdose (Stein et al., 2017). Likewise, studies have reported that higher ACE scores are associated with increased risk of suicidal ideation and a suicide attempt (Dube et al., 2001; Thompson et al., 2019). Based on previous research, it is hypothesized that perceived stressful life events will be associated with SI/SA while ACE domain scores will be an overlapping factor in both SI/SA (Chen et al., 2010) and the unintentional overdose groups (Stein et al., 2017). Furthermore, subjects with more recent episodes of SIB will represent the group with a more severe behavioral profile (i.e., SI/SA/OD) who engage in more high-risk behaviors and require higher levels of care.

\subsection{Methods}

$\underline{\text { Study Design }}$

This was a cross-sectional study conducted in a subset of subjects recruited as part of a larger inpatient study. The original study recruited inpatients with OUD to test the acceptability of an integrated suicide and overdose screen.

Setting

A convenience sample of patients with an OUD was recruited from two treatment programs associated with a university-based health care system in West Virginia. One facility is an 18-bed acute inpatient dual-diagnosis unit that treats individuals who: 1) need medically managed detoxification and 2) are a danger to themselves or others (i.e., suicidal ideation/suicide attempt and/or homicidal ideation or actions). The second site is a 28-day residential addiction treatment program. 


\section{$\underline{\text { Data Collection }}$}

\section{Study Sample.}

The parent study recruited subjects $(\mathrm{n}=113)$ between February 2019 and October 2019. Patients were included in the study if they had an opioid use disorder, were psychiatrically stable, and were over the age of 18 years old.

Participation was voluntary, and subjects were not compensated for study participation. Only subjects that self-reported suicidal ideation in the past two weeks, an overdose in the past six months, and/or a suicide attempt in the past six months, were included in this study $(\mathrm{n}=66)$.

\section{$\underline{\text { Measures }}$}

\section{Administrative Clinical Data}

Demographic (sex, race, ethnicity, education, and number of lifetime arrests), clinical characteristics, substance use history, ACE sub-scores, and ACE total score were extracted from an administrative clinical database (Winstanley et al., 2020). Race was recoded into a dichotomous variable (white/non-white); because the sample was predominantly white and had little variability. Employment was also recoded into a dichotomous variable (employed full/parttime or unemployed). Education was recoded into a categorical variable (less than high school education, high school graduate/GED, or some college/college graduate). Substance use history was recoded as lifetime use (yes/no) for tobacco, alcohol, cocaine, marijuana, heroin, prescription opioids, illicit/non-medical use of buprenorphine, methamphetamines, and benzodiazepines. This history also included age of first use for each substance endorsed by the subject, and lifetime injection drug use. 


\section{Adverse Childhood Experiences.}

The clinical administrative database included a modified version of the adverse childhood experiences (ACEs) questionnaire (Winstanley et al. 2020). ACEs measure different types of childhood adversities experienced before age 18 and higher scores are associated with overdose and suicidal behaviors (Chen et al., 2010; Stein et al., 2017; Thompson et al., 2019). The modified ACE has 17 items that were grouped into the following categories of adverse experiences: mental health problems; alcohol or drug use; emotional, physical or sexual abuse; neglect; physical or emotional abuse by a significant other (teen dating abuse), and family dissolution. A total ACE score was generated by summing the number of categories endorsed. Self-Harm Assessment

This assessment was a 30-minute face-to-face interview, which included information on drugs used prior to last overdose, past year stressful life events, self-harm behaviors, suicidal ideation, and suicide attempts. Global mental health was assessed using the Patient Reported Outcomes Measurement Information System (PROMIS) item, "In general, how would you rate your mental health, including your mood and ability to think?" This was rated on a 5-point Likert scale from poor to excellent (Cella et al., 2010). Due to the distribution of responses, "excellent," "very good," and "good were collapsed into one category to create the following three categories: 1) "good,” 2) "fair," and 3) "poor.” Two items from the Beck scale for suicidal ideation (Beck et al., 1979). The first item was "how you have been feeling over the past week, including today" and the response options were 1) "I have a moderate to strong wish to live," 2) "I have a weak wish to live," or 3) "I have no wish to live" and it was recoded as moderate/strong wish to live or weak/no wish to live. For the second item asked how he or she was feeling in the past week and the response options were 1) "I would try to save my life if I 
found myself in a life-threatening situation," 2) "I would take a chance on life or death," or 3) "I would not take the steps necessary to avoid death if I found myself in a life-threatening situation."

\section{Overdose Assessment}

For this study, an opioid overdose was defined as "when you cannot breathe and cannot wake up without medical help." Irrespective of when the overdose occurred, subjects who endorsed ever having an overdose, were asked 10 questions that queried details about their most recent overdose. They were asked to report their age of first overdose and the number of overdoses they have experienced in their lifetime. Subjects were asked for the date of their most recent overdose, the location where the overdose occurred and, if it was the first time they experienced an overdose (yes/no). Subjects were also asked if their most recent overdose was an accident (yes/no). They were further asked if they used alcohol (yes/no), prescription drugs (yes/no), street drugs (yes/no), and/or over the counter medications (yes/no) in the 24-hours prior to their overdose. For each category that the subject reported "yes," he/she was asked to specify the name of the substance used. Drugs and medications used were categorized as antidepressants, antipsychotics, benzodiazepines, cocaine, prescription opioids, heroin, methamphetamines, and sedatives. Subjects were asked if naloxone was used to revive them (yes/no); did anyone do anything to help you when you last overdosed (yes/no); if measures were taken to help you during your last overdose, what were these helpful measures (open-ended); if counseling or overdose education was provided after the overdose (yes/no); and if the patient would like any additional services (yes/no). 


\section{Life Events Checklist}

Stressful life events were measured using a modified Life Events Checklist (LEC), which measures life events that adults often find difficult or stressful in the past year. These events included experiencing a natural disaster, fire or explosion, transportation accident, serious accident, exposure to a toxic substance, physical assault, assault with a weapon, sexual assault, unwanted sexual experience, combat exposure, captivity, severe human suffering, sudden violent death, sudden accidental death, serious injury or harm the person inflicted on another person, and/or any other very stressful event or experience (Weathers et al., 2013). The original 17-item LEC was modified by the inclusion of an additional item on dissolution of a romantic relationship in the past year. This item was added because research has found that ending of a romantic relationship is a stressful event that is associated with suicide attempt (Dragisic et al., 2015; Heale et al., 2003). The LEC has five response items and subjects were instructed to select all options that applied for each event over the course of their lifetime. For this study, subjects were instructed to only consider these events for the past year instead of over the course of their lifetime and to only choose one response instead of choosing all responses that apply (Weathers et al., 2013). For the purposes of this study, a total score was generated to reflect the number of stressful life events in the past year. Each response was assigned a numeric value: (a) it happened to you personally $=3$, (b) you witnessed it happen to someone else $=2$, (c) you learned about it happening to someone close to you=1, (d) you're not sure if it fits (coded as missing), or (e) it doesn't apply to you $=0$. For the questions on sudden violent death (for example, a homicide or suicide) and sudden accident death, witnessing it happen to someone else was scored as 3 and learning about it as 2 , since these events could not directly happen to the subject or participant. The LEC items were summed to generate a total score, which ranged from 0-54. 


\section{Self-Injurious Behaviors Assessment}

The non-suicidal self-injury section of the Self-Injurious Thoughts and Behaviors Interview (SITBI-SF) (Nock et al., 2007) was used to measure SIB and evaluate lifetime suicidal ideation. Lifetime suicidal ideation was measured using the first three questions from the SITBISF suicidal ideation section. Self-injurious behaviors were measured using all questions from the SITBI-SF included in the non-suicidal self-injury portion of the interview. The questions related to suicide plan, attempt, and gesture were omitted for the purposes of this research study.

\section{Suicidal Behaviors Assessment}

Additional suicidal behaviors were evaluated using the suicide behaviors questionnairerevised form (SBQ-R) (Osman et al, 2001). This tool uses four questions that ask patients if they have ever thought about or attempted suicide, how often they have thought about suicide in the past year, if they ever told another person they might attempt suicide, and how likely is it they would attempt suicide in the future. For the last question, the response options are never, no chance at all, rather unlikely, unlikely, likely, rather likely, and very likely. For analytic purposes, the categories of never/no chance at all, rather unlikely/unlikely, and likely/rather likely were collapsed into four response options. SBR-Q scoring is calculated using the original responses, without alteration of the response categories. This tool is used to identify suicide risk and risk behaviors, yielding a score ranging from 3-18, among inpatients (Osman et al., 2001), A score of greater than or equal to eight classified inpatients as having increased suicide risk with 80\% sensitivity (true-positivity) and 91\% specificity (true-negativity).

Data obtained during the patient interview was entered into a REDCap (Remote Electronic Data Capture) database, using an encrypted tablet computer. If an immediate crisis was identified (i.e., patient discloses intent to harm themselves, others, or discloses current 
suicidal ideation/plan), the attending physician and charge nurse were notified immediately. The duration of the total screening and interview time was approximately 40-50 minutes.

\section{$\underline{\text { Data Analysis }}$}

The outcome variable was based on patient self-report of suicidal ideation in the past two weeks, overdose in the past six months, and/or suicide attempt in the past six months. This information was used to create the following mutually exclusive categories:1) unintentional overdose(s) only (no suicidal ideation in the past two weeks or attempt in the past six months); 2) suicidal ideation (past two weeks), suicide attempt (past six months) (SI/SA); and 3) unintentional overdose, suicidal ideation, and suicide attempt(s) including intentional overdose (SI/SA/OD). Descriptive statistics were used to summarize the sociodemographic and clinical characteristics of the sample. Medians were reported for variables that were not normally distributed, except for age. Statistical analysis employed using Pearson's chi-square tests for categorical variables, ANOVAs for means, and Kurskal-Wallis H-test to compare medians with Dunn's test post-hoc analysis.

Bivariable analysis was conducted with 47 variables, including ACE category scores, total ACE domain score, each item of the LEC, LEC-total score, SIB behavior measures, lifetime overdose(s), substances used 24-hours prior to overdose, suicidal behaviors, hopelessness, and social support. Two multinomial logistic regression models were built (adjusted and unadjusted) with the SI/SA group as the base outcome. The unadjusted multinomial logistic regression examined ace total category score, past year work accident, past year sexual assault, past year combat exposure, hitting, depression in the past two weeks, and hopelessness in the past two weeks. The second model was an adjusted model that controlled for age and sex while examining ACE total category score, past year work accident, past year sexual assault, past year combat 
exposure, hitting, depression in the past two weeks, hopelessness in the past two weeks, and global social support score. Data analysis was conducted using Stata/SE 15.0 analytical software (StataCorp, 2017).

\subsection{Results}

The sample included 66 subjects that endorsed suicidal ideation in the past two weeks, an overdose in the past six months, and/or a suicide attempt in the past six month; $19.7 \%(n=13)$ reported unintentional OD, 50.0\% $(n=33) \mathrm{SI} / \mathrm{SA}$ and $30.0 \%(\mathrm{n}=20) \mathrm{SI} / \mathrm{SA} / \mathrm{OD}$. The overall sample was predominantly male (65.2\%), white (93.4\%), non-Hispanic (93.4\%), unemployed (74.2\%), and had a high school education (50.0\%). There were no statistically significant group differences in the demographic characteristics (data not shown).

With respect to lifetime substance use, the only statistically significant differences were for cocaine and prescription opioid use. The OD group more likely to have used lifetime cocaine use during their lifetime (100.0\%) compared to either of the other two groups ( $\mathrm{SI} / \mathrm{SA}=72.7 \%$, $\mathrm{SI} / \mathrm{SA} / \mathrm{OD}=95.0 \%, \mathrm{p}=0.02)$. For the overall sample, the mean age of initiation of prescription opioids was 18.4 years $(\mathrm{SD}=7.4)$. The mean age of initiation of heroin use was 24.7 years $(\mathrm{SD}=9.8)$. The $\mathrm{SI} / \mathrm{SA}$ group was more likely to have used prescription opioids during their lifetime (97.0\%) compared to the unintentional OD group (69.2\%) or the SI/SA/OD group $(90.0 \%, p=0.03)$. There were no statistically significant group differences in age of onset of drug use (see Table 4.1). Age of first prescription opioid use preceded onset of heroin use for all groups (Table 4.1). There groups did not differ in terms of recent (past month) drug use, injection drug use, age of first overdose or number of overdoses. With respect to substances used in the 24-hours prior to the most recent overdose, the five most commonly used substances were 
heroin (67.4\%), benzodiazepines (21.7\%), alcohol (20.0\%), prescription opioids (19.6\%), and methamphetamine $(15.2 \%)$.

Only two substances reported in the 24-hours prior to overdose were found to be statistically significantly different between groups: antipsychotics and heroin. In the 24-hours prior to their most recent overdose, $30.7 \%$ of the SI/SA group reported using an anti-psychotic medication compared to $7.7 \%$ in the OD group and no one in the SI/SA/OD group reported using anti-psychotic medications prior to their last overdose ( $\mathrm{p}=0.02)$. For the OD group, $92.3 \%$ reported using heroin prior to their most recent overdose compared to $30.7 \%$ of subjects in the SI/SA and $75.0 \%$ in the SI/SA/OD group $(\mathrm{p}<0.01)$ (see Table 4.1 ).

The SI/SA group were more likely to report physical abuse (93.9\%), than either the OD group $(46.2 \%)$ or the SI/SA/OD group $(85.0 \% ; \mathrm{p}<0.01)$. Teen dating violence was reported more frequently by subjects in the SI/SA (93.9\%), than either the unintentional OD group (46.2\%), or the SI/SA/OD group $(85.0 \% ; \mathrm{p}<0.01)$. Overall, the SI/SSA group had a higher mean ACE score (mean=5.5) compared to either the OD group (mean=4.0) or the SI/SA/OD group (mean=5.0, $\mathrm{p}=0.04$ ). No statistically significant differences were observed with respect to cumulative mean past year stressful life event (LEC score) scores or individual LEC items (Table 4.2).

The overall sample had a median number of lifetime SIBs of 15 . The OD group reported more lifetime SIBs (median=20) compared to the SI/SA group (median=45, $\mathrm{p}=0.05)($ Table 4.3). No statistically significant difference was observed for mean age of first SIB, last occurrence of SIB, or type of SIB. with $40.6 \%$ of subjects in the SI/SA group reporting hitting themselves on purpose compared to $30.0 \%$ of the SI/SA/OD group and $7.7 \%$ of subjects in the unintentional OD group. 
The unintentional OD group was more likely to report a moderate/strong wish to live (100.0\%), compared to either the SI/SA (63.6\%) or SI/SA/OD group $(65.0 \%, \mathrm{p}=0.02)$ (Table 4.4). Last occurrence of suicidal ideation more frequently occurred in the past month for the SI/SA (90.9\%) and SI/SA/OD groups (68.4\%) whereas more of the unintentional OD group reported suicidal ideation more than a year ago $(50.0 \%, \mathrm{p}=0.02)$. There was no statistically significant difference in terms of mean age of first suicidal ideation or median lifetime episodes of suicidal ideation. There was however, a statistically significant difference between groups for last occurrence of suicidal ideation $(\mathrm{p}<0.01$; Table 4.4$)$. In the past month $75.8 \%$ of subjects reported suicidal ideation in the past month. The largest portion of subjects reporting past month suicidal ideation were those in the SI/SA group (90.9\%), followed by the SI/SA/OD group (68.4\%), and the unintentional OD group (16.7\%; Table 4.4). Results of suicidal behaviors are reported in Table 4.4 and the OD group never attempted suicide; $69.7 \%$ of the SI/SA group and $45.0 \%$ of SI/SA/OD group reported lifetime suicide attempt with hope to die $(\mathrm{p}<0.01$; Table 4.4$)$.

The following covariates were statistically significant in the bivariable regression models: ACE score, work accidents (past year), sexual assault (past year), past year combat exposure, (past year), hitting (lifetime), depression (past two weeks), hopelessness (past two weeks), and global social support measures (data not shown). ACE score $(p=0.05)$, work accident $(p<0.01)$, sexual assault $(p<0.01)$, combat exposure $(p<0.01)$, and hopelessness $(p=0.03)$ remained statistically significant in the unadjusted regression model between the OD and SI/SA groups. For subjects with SI/SA/OD there was a statistically significant difference for past year work accidents $(\mathrm{p}=0.02)$ and combat exposure $(\mathrm{p}<0.01)$ when comparted to subjects with SI/SA (see Table 4.5). 
In the adjusted (adjusting for age and sex) multivariable model past year work accidents $(\mathrm{p}=0.02)$ and combat exposure $(\mathrm{p}<0.01)$ for subjects in the SI/SA/OD group compared to the SI/SA group (see Table 4.5). The OD group compared to the SI/SA group for ACE score $(\mathrm{p}=0.03)$, work accident $(\mathrm{p}<0.01)$, sexual assault $(\mathrm{p}<0.01)$, and combat exposure $(\mathrm{p}<0.01)$.

\subsection{Discussion}

This study found that clinical characteristics and life event differences do exist between treatment-seeking adults with OUD with history of SI/SA/OD. While the national focus is on unintentional overdoses among individuals with OUD, this study found $50 \%$ had a history of suicidal ideation or attempts and $19.7 \%$ had a history of only unintentional overdose. This indicates that greater attention be paid to factors contributing to co-morbid OUD and suicidal behaviors. Specifically, as it applies to substances used prior to overdose, adverse childhood experiences (ACEs), history of stressful life events, and self-injurious behaviors.

Consistent with previous research (Vingoe et al., 1999; Maloney et al., 2007; Neale, 2000), heroin is infrequently used as a method of suicide or in self-reported intentional overdoses. Even in samples where heroin was reported in the overdose, it was significantly more likely to be used in an accidental overdose than a deliberate overdose (Maloney et al., 2007; Neale, 2000). Another finding, consistent with previous research, is that subjects with SI/SA/OD and SI/SA reported a higher prevalence of lifetime prescription opioid use than subjects with unintentional overdose (Hakansson et al. 2010; Roy 2002). However, rates of antipsychotic use could in part be explained by the large number of subjects recruited from an acute inpatient setting. Contrary to previous studies (Ilgen et al, 2009; Roy 2002; Roy 2010), this study found that subjects with unintentional overdose more frequently reported lifetime use of cocaine compared to subjects in the group with SI/SA. However, use of cocaine among individuals in the unintentional OD group 
contradicts previous studies which found significant associations between cocaine use among individuals with OUD who attempted suicide (Ilgen et al, 2009; Roy 2002; Roy 2010). In our sample, the lack of association between cocaine and suicide attempt may be explained by lack of racial heterogeneity. Additionally, the SI/SA group reports overall less use of methamphetamine, cocaine, and heroin compared to the unintentional OD group, but higher use of antidepressants, antipsychotics, and benzodiazepines. Among subjects in the current study, in addition to heroin, the most frequently reported drug taken in the 24-hours prior to overdose among subjects with SI/SA was antipsychotics. Overall patterns of substance use may be indicative of time in treatment, more utilization of mental and/or behavioral healthcare services, which may limit access to other illicit substances. However, length of stay and healthcare utilization were not measured in the current study. Although specific mental health diagnoses were not recorded as part of the current study, considering the recruitment setting (acute inpatient treatment and 28day residential treatment programs), it is possible that participants may receive psychiatric medication interventions where the medication can be used in a suicide attempt (Bohnert \& Ilgen, 2019).

Individuals in the SI/SA group had higher ACE scores and were more likely to have experienced physical abuse or teen dating violence before the age of 18 , which is consistent with previous research (Hakansson et al., 2006; Roy, 2002; Darke et al, 2012). While previous research has demonstrated a connection between childhood sexual abuse and the risk of overdose among individuals with OUD (Molnar et al., 2001; Roy, 2002), interestingly our study found that sexual abuse in the past year more strongly associated with unintentional overdose. These patterns of abuse both inside and outside the home could be associated with low levels of family 
support, high levels of hopelessness, and perpetuating cycles of depression and hopelessness that contribute to resulting suicidal behaviors (Bradvik et al., 2007; Chen et al., 2009).

Moreover, self-injurious behaviors are one of the most important risk factors for future suicide and are often even more predictive than a history of suicidal ideation (Cavanagh et al.,2003). As seen with previous research (Darke et al., 2012), this study found that the mean age of onset for SIB (14 years old) preceded the mean age of onset of suicidal ideation (21 years old). Most research reports first SIB in early adolescence (Maloney et al., 2010) while this was true for the unintentional overdose group, the SI/SA and SI/SA/OD groups had mean age of onset of SIB occurring later at age 16.7 and 16.9 respectively. Furthermore, more individuals in the SI/SA group engaged in SIBs in the past month compared to either the SI/SA/OD group or the unintentional OD group where no participants reported any SIBs in the past month. While these results were not statistically significant, they are clinically meaningful in that participants engaging in SIB also reported suicidal behaviors more frequently than those without any SIBs in the prior month.

\section{Limitations}

We acknowledge this study has several limitations. First, despite specification of opioid overdose as "decreased respiratory depression," subjects reported non-opioid related overdoses. Lifetime suicidal ideation was assessed using retrospective self-reporting and recall bias may be problematic. Recall bias could have also influenced reporting of substances used in the 24-hours prior to the most recent overdose, since the time period between interview and date of the subject's last overdose was variable. Additionally, length of stay and healthcare utilization were not measured in the current study. This limited our ability to examine or extrapolate any causal relationships between past 30-day substance use with outcome behaviors such as overdose, 
suicide attempt, suicidal ideation, and self-injurious behaviors. Likewise, the sample size was small limiting this study to be conducted as exploratory research and hence the power to detect group differences was not calculated. Our sample was predominantly white and included residents residing in a non-urban Appalachian area, which may limit generalizability regarding different demographic groups and subjects receiving outpatient OUD treatment.

\section{Future Research}

There are several future directions suggested by this research. First, the results such more in-depth investigation of overdose typologies that differentiate overdose intentionality and specification of substances used. Prospective studies are needed to understand causality and model the dynamics of intentionality, as well as account for how impulsivity may moderate the association. Moreover, unintentional overdoses and suicidal behaviors were found to be associated with wish to live and SIB. These results have the potential to inform clinical interventions and harm reduction strategies as it may help identify intentionality. These patients may utilize multiple methods for suicide attempts further complicating the determination of overdose intentionality. This issue warrants further investigation not only from an intentionality perspective, but also to develop more effective harm reduction strategies.

\subsection{Conclusions}

Results from this study partially elucidated differences clinical and behavioral differences between OUD patients with a history of unintentional overdose versus suicidal ideation/attempt. Whereas, subjects with a history of SI/SA/OD represent a diverse group with potentially greater severity that would likely benefit from multimodal intervention. Despite, differences in substance use that distinguish intentional from unintentional overdoses, future research needs to focus on systematically addressing overdose typologies. Overall, this study highlights the 
importance of addressing the contribution of history of self-injury, types of substances used the day of overdose, recent life stressors, and adverse childhood experiences for both overdose and suicide among patients with opioid use disorders.

\subsection{Acknowledgements}

Funding Source: ANS received funding from the National Institute of General Medical Sciences of the National Institutes of Health (1 T32 GM132494). 


\begin{tabular}{|c|c|c|c|c|c|}
\hline & $\begin{array}{c}\begin{array}{c}\text { Overall Sample } \\
(n=66)\end{array} \\
\end{array}$ & $\begin{array}{c}\text { Unintentional OD }^{1} \\
(n=13)\end{array}$ & $\begin{array}{l}\text { SISA }^{2} \\
(n=33)\end{array}$ & $\begin{array}{c}\text { SI/SA/OD } \\
(\mathbf{n}=\mathbf{2 0})\end{array}$ & p-value \\
\hline \multicolumn{6}{|l|}{ Characteristics } \\
\hline $\begin{array}{l}\text { Mean number substances used (Past } 30 \text { days), } \\
(\mathrm{SD})^{\dagger}\end{array}$ & $2.5(1.5)$ & $2.2(2.0)$ & $2.5(1.2)$ & $2.7(1.7)$ & 0.64 \\
\hline Ever Inject Drugs (\%Yes, Lifetime) & 75.8 & 84.6 & 66.7 & 85.0 & 0.26 \\
\hline Mean Age First IDU (SD) ${ }^{+}$ & $24.9(9.2)$ & $23.5(8.5)$ & $24.5(9.0$ & $26.3(10.1)$ & 0.72 \\
\hline Median Number Overdoses & 3.0 & 3.0 & 3.0 & 1.5 & 0.71 \\
\hline Mean Age First Overdose (SD) ${ }^{+}$ & $27.4(11.0)$ & $29.9(11.8)$ & $25.7(9.9)$ & $26.7(11.5)$ & 0.54 \\
\hline \multicolumn{6}{|l|}{ Substances Used 24 hours Prior to OD (\% Yes) } \\
\hline Alcohol & 20.0 & 23.1 & 18.8 & 20.0 & 0.92 \\
\hline Antidepressants & 10.9 & 7.7 & 23.1 & 5.0 & 0.36 \\
\hline Antipsychotics & 10.9 & 7.7 & 30.7 & 0.0 & 0.02 \\
\hline Benzodiazepines & 21.7 & 23.1 & 15.4 & 25.0 & 0.90 \\
\hline Cocaine & 13.0 & 7.7 & 0.0 & 25.0 & 0.15 \\
\hline Prescription Opioids & 19.6 & 15.4 & 23.1 & 20.0 & 1.00 \\
\hline Heroin & 67.4 & 92.3 & 30.7 & 75.0 & $<0.01$ \\
\hline Methamphetamine & 15.2 & 23.1 & 7.7 & 15.0 & 0.69 \\
\hline Sedatives & 2.2 & 0.0 & 0.0 & 5.0 & 1.00 \\
\hline Naloxone to reverse (\% Yes) & 52.4 & 76.9 & 41.7 & 41.2 & 0.11 \\
\hline \multicolumn{6}{|c|}{ 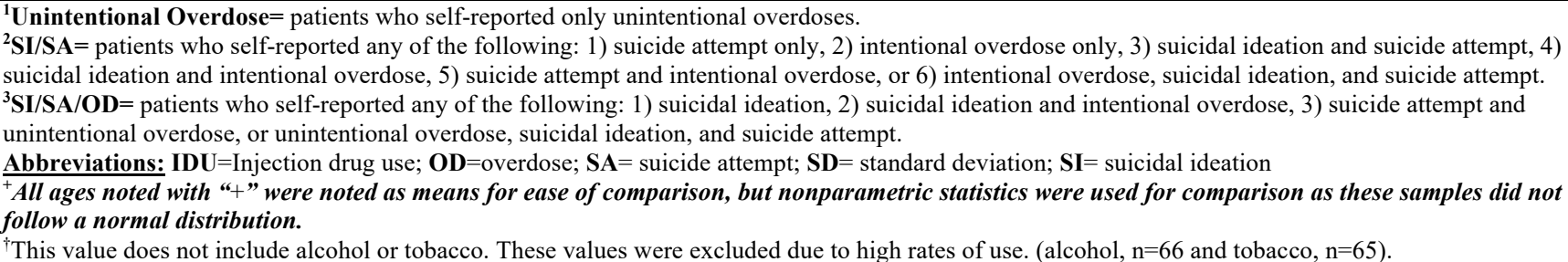 } \\
\hline
\end{tabular}




\begin{tabular}{|c|c|c|c|c|c|}
\hline & $\begin{array}{c}\text { Overall Sample } \\
(n=66)\end{array}$ & $\begin{array}{c}\text { Unintentional OD }^{1} \\
(n=13)\end{array}$ & $\begin{array}{l}\text { SISA }^{2} \\
(n=33)\end{array}$ & $\begin{array}{c}\mathrm{SI} / \mathrm{SA} / \mathbf{O D}^{3} \\
(\mathbf{n}=\mathbf{2 0})\end{array}$ & p-value \\
\hline \multicolumn{6}{|l|}{ ACE Domains (\% Yes) } \\
\hline Mental Health Problems & 63.6 & 38.5 & 75.8 & 60.0 & 0.06 \\
\hline Alcohol or Drug Use & 77.3 & 61.5 & 81.8 & 80.0 & 0.37 \\
\hline Emotional Abuse & 71.2 & 46.2 & 75.8 & 80.0 & 0.09 \\
\hline Physical Abuse & 81.8 & 46.2 & 93.9 & 85.0 & $<0.01$ \\
\hline Teen Dating Violence* & 81.8 & 46.2 & 93.9 & 85.0 & $<0.01$ \\
\hline Sexual Abuse & 51.5 & 38.5 & 63.6 & 40.0 & 0.17 \\
\hline Neglect & 93.9 & 100.0 & 97.0 & 85.0 & 0.12 \\
\hline Family Dissolution & 65.2 & 69.2 & 69.7 & 55.0 & 0.52 \\
\hline Total mean ACE domain score (SD) & $5.0(1.7)$ & $4.0(1.7)$ & $5.5(1.4)$ & $5.0(1.9)$ & 0.04 \\
\hline Total mean LEC score (SD) & $11.7(8.5)$ & $10.8(6.9)$ & $11.7(8.3)$ & $12.1(10.0)$ & 0.90 \\
\hline
\end{tabular}

${ }^{1}$ Unintentional Overdose $=$ patients who self-reported only unintentional overdoses.

${ }^{2} \mathbf{S I} / \mathbf{S A}=$ patients who self-reported any of the following: 1) suicide attempt only, 2) intentional overdose only, 3) suicidal ideation and suicide attempt, 4) suicidal ideation and intentional overdose, 5) suicide attempt and intentional overdose, or 6) intentional overdose, suicidal ideation, and suicide attempt.

${ }^{3}$ SI/SA/OD= patients who self-reported any of the following: 1) suicidal ideation, 2) suicidal ideation and intentional overdose, 3) suicide attempt and unintentional overdose, or unintentional overdose, suicidal ideation, and suicide attempt.

*Define as physical or emotional abuse experienced by a significant other before the age of 18 years old.

Abbreviations: $\mathbf{A C E}=$ adverse childhood experiences; $\mathbf{L E C}=$ life events checklist; $\mathbf{O D}=$ overdose; $\mathbf{S A}=$ suicide attempt; $\mathbf{S D}=$ standard deviation; $\mathbf{S I}=$ suicidal ideation 


\begin{tabular}{|c|c|c|c|c|c|}
\hline & $\begin{array}{c}\text { Overall Sample } \\
(n=66)\end{array}$ & $\begin{array}{c}\text { Unintentional } \text { OD }^{1} \\
(n=13)\end{array}$ & $\begin{array}{l}\text { SISA }^{2} \\
(\mathrm{n}=33)\end{array}$ & $\begin{array}{c}\mathbf{S I} / \mathbf{S A} / \mathbf{O D}^{3} \\
(\mathbf{n}=\mathbf{2 0})\end{array}$ & p-value \\
\hline Wish to live & & & & & 0.02 \\
\hline Moderate/Strong & 71.2 & 100.0 & 63.6 & 65.0 & \\
\hline Weak/None & 28.3 & 0.0 & 36.4 & 35.0 & \\
\hline Life Threatening Situation & & & & & 0.71 \\
\hline Would try to save my life & 60.6 & 76.9 & 54.6 & 60.0 & \\
\hline Would take a chance on life/death & 30.3 & 23.1 & 33.3 & 30.0 & \\
\hline Would not avoid death & 9.1 & 0.0 & 12.1 & 10.0 & \\
\hline History of SIB (\% Yes) & 37.9 & 23.1 & 42.4 & 40.0 & 0.56 \\
\hline Mean age first SIB + & $16.4(6.8)$ & $14.0(1.0)$ & $16.7(8.3)$ & $16.9(5.4)$ & 0.66 \\
\hline Median lifetime episodes SIB & 15.0 & 20.0 & 45.0 & 5.0 & 0.05 \\
\hline Last occurrence of SIB & & & & & 0.73 \\
\hline In past month & 28.0 & 0.0 & 35.7 & 25.0 & \\
\hline Past 2-12 months & 40.0 & 33.3 & 42.9 & 37.5 & \\
\hline More than a year ago & 32.0 & 66.7 & 21.4 & 37.5 & \\
\hline \multicolumn{6}{|l|}{ SIB Behaviors (\% Yes) } \\
\hline Cutting & 33.9 & 15.4 & 40.6 & 35.0 & 0.30 \\
\hline Hitting oneself on purpose & 30.8 & 7.7 & 40.6 & 30.0 & 0.08 \\
\hline Hair pulling & 18.5 & 7.7 & 28.1 & 10.0 & 0.22 \\
\hline Self-tattooing & 13.9 & 7.7 & 15.6 & 15.0 & 0.90 \\
\hline Picked a wound & 21.5 & 7.7 & 31.3 & 15.0 & 0.20 \\
\hline Burning & 15.4 & 7.7 & 21.9 & 10.0 & 0.48 \\
\hline Inserted objects under nails or skin & 4.6 & 0.0 & 9.4 & 0.0 & 0.30 \\
\hline Biting & 9.2 & 0.0 & 15.6 & 5.0 & 0.32 \\
\hline Picked areas of body to point of drawing blood & 13.9 & 0.0 & 18.8 & 15.0 & 0.32 \\
\hline Scraped skin & 10.8 & 0.0 & 15.6 & 10.0 & 0.38 \\
\hline "Erased" skin to the point of drawing blood & 6.2 & 0.0 & 6.3 & 10.0 & 0.66 \\
\hline Received medical treatment for SIB (\% Yes) & 40.0 & 33.3 & 42.9 & 37.5 & 1.00 \\
\hline \multicolumn{6}{|c|}{ 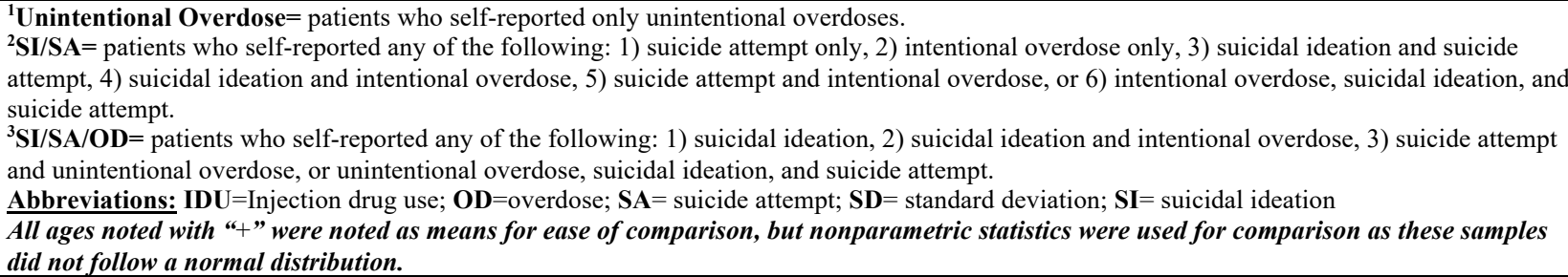 } \\
\hline
\end{tabular}




\begin{tabular}{|c|c|c|c|c|c|}
\hline & $\begin{array}{c}\text { Overall } \\
\text { Sample } \\
(n=66)\end{array}$ & $\begin{array}{c}\text { Unintentional } \\
\text { OD }^{1} \\
(\mathbf{n}=13)\end{array}$ & $\begin{array}{l}\text { SISA }^{2} \\
(n=33)\end{array}$ & $\begin{array}{c}\mathrm{SI} / \mathrm{SA} / \mathbf{O D}^{3} \\
(\mathbf{n}=\mathbf{2 0})\end{array}$ & p-value \\
\hline Mean Age first suicidal ideation (SD)+ & $21.0(12.2)$ & $23.3(11.9)$ & $20.6(13.5)$ & $20.8(10.1)$ & 0.64 \\
\hline Median episodes suicidal ideation & 10.0 & 7.0 & 15.0 & 10.0 & 0.45 \\
\hline Last occurrence of suicidal ideation & & & & & 0.02 \\
\hline In the past month & 82.7 & 16.7 & 90.9 & 68.4 & \\
\hline Past 2-12 months & 9.6 & 33.3 & 9.1 & 10.5 & \\
\hline More than one year ago & 7.7 & 50.0 & 0.0 & 21.1 & \\
\hline Suicide Attempt(s) (\% Yes) & & & & & $<0.01$ \\
\hline Never & 28.8 & 100.0 & 9.1 & 15.0 & \\
\hline Brief, passing thought & 10.6 & & 12.1 & 15.0 & \\
\hline I had a plan once, but did not try & 6.1 & & 6.1 & 10.0 & \\
\hline I had a plan once, and really wanted to die & 0.0 & & 0.0 & 0.0 & \\
\hline I have attempted to kill myself, but did not want to die & 6.1 & & 3.0 & 15.0 & \\
\hline I have attempted to kill myself, and really hoped to die & 48.5 & & 69.7 & 45.0 & \\
\hline Frequency of Suicidal Ideation (\% Yes) & & & & & 0.55 \\
\hline Never/Rarely & 7.5 & & 3.9 & 14.3 & \\
\hline Sometimes & 7.5 & & 3.9 & 7.1 & \\
\hline Often/Very Often & 85.0 & & 88.5 & 78.6 & \\
\hline Disclosure of Suicide Attempt (\% Yes) & & & & & 0.91 \\
\hline No & 50.0 & & 53.9 & 42.9 & \\
\hline Yes, at one time, but did not want to die & 5.0 & & 3.9 & 7.1 & \\
\hline Yes, at one time, and really wanted to die & 12.5 & & 11.5 & 14.3 & \\
\hline Yes, more than once, but did not want to die & 2.5 & & 3.9 & 0.0 & \\
\hline Yes, more than once, and really wanted to die & 30.0 & & 26.9 & 35.7 & \\
\hline Likelihood of future suicide attempt (\% Yes) & & & & & 0.23 \\
\hline Never/No Chance & 32.5 & & 30.8 & 35.7 & \\
\hline Unlikely & 37.5 & & 30.8 & 50.0 & \\
\hline Likely & 15.0 & & 15.4 & 14.3 & \\
\hline Very Likely & 15.0 & & 23.1 & 0.0 & \\
\hline Mean Suicide Behaviors Questionnaire (SBQ-R) Total Score ${ }^{+}$ & $8.2(6.1)$ & & $10.8(5.5)$ & $8.7(5.5)$ & 0.15 \\
\hline \multicolumn{6}{|c|}{$\begin{array}{l}{ }^{1} \text { Unintentional Overdose }=\text { patients who self-reported only unintentional overdoses. } \\
{ }^{2} \mathrm{SI} / \mathrm{SA}=\text { patients who self-reported any of the following: 1) suicide attempt only, 2) intentional overdose only, 3) suicidal ideation and suicide } \\
\text { attempt, 4) suicidal ideation and intentional overdose, 5) suicide attempt and intentional overdose, or } 6 \text { ) intentional overdose, suicidal ideation, and } \\
\text { suicide attempt. }\end{array}$} \\
\hline
\end{tabular}




\begin{tabular}{|c|c|c|c|c|c|c|}
\hline & \multicolumn{6}{|c|}{ Unadjusted Analyses } \\
\hline & \multicolumn{3}{|c|}{ Unintentional OD } & \multicolumn{3}{|c|}{ SI/SA/OD } \\
\hline Variable & Coefficient & $95 \% \mathrm{CI}$ & p-value & Coefficient & $95 \% \mathrm{CI}$ & p-value \\
\hline ACE total domain score & -0.5 & $-1.1,-0.01$ & 0.05 & -0.4 & $-0.9,0.1$ & 0.15 \\
\hline Work accident (past year) & -15.1 & $-18.3,-17.2$ & $<0.01$ & 2.1 & $0.3,3.9$ & 0.02 \\
\hline Sexual assault (past year) & -19.2 & $-21.2,-17.2$ & $<0.01$ & -0.9 & $-2.9,1.1$ & 0.38 \\
\hline Combat exposure (past year) & -18.2 & $-21.6,-14.9$ & $<0.01$ & -18.2 & $-21.1,-15.3$ & $<0.01$ \\
\hline Hitting & 0.4 & $-2.1,2.9$ & 0.74 & -0.2 & $-2.0,1.6$ & 0.9 \\
\hline Depression (past two weeks) & -0.4 & $-3.4,2.5$ & 0.78 & -0.8 & $-4.0,2.4$ & 0.6 \\
\hline \multirow[t]{3}{*}{ Hopelessness (past two weeks) } & -3.0 & $-5.9,-0.2$ & 0.03 & -0.2 & $-2.6,2.3$ & 0.9 \\
\hline & \multicolumn{6}{|c|}{ Adjusted Analyses } \\
\hline & \multicolumn{3}{|c|}{ Unintentional OD } & \multicolumn{3}{|c|}{ SI/SA/OD } \\
\hline Variable & Coefficient & $95 \%$ CI & p-value & Coefficient & $95 \% \mathrm{CI}$ & p-value \\
\hline Age & -0.1 & $-0.2,0.03$ & 0.15 & -0.1 & $-0.2,-0.01$ & 0.02 \\
\hline Sex & -0.7 & $-2.5,1.1$ & 0.43 & -0.1 & $-1.6,1.3$ & 0.87 \\
\hline Work accident (past year) & -13.8 & $-17.0,-10.7$ & $<0.01$ & 2.1 & $0.3,3.9$ & 0.02 \\
\hline Sexual assault (past year) & -17.0 & $-19.6,-14.5$ & $<0.01$ & -0.7 & $-2.6,1.2$ & 0.45 \\
\hline Combat exposure (past year) & -15.1 & $-20.3,-9.9$ & $<0.01$ & -16.7 & $-19.2,-14.1$ & $<0.01$ \\
\hline Hitting & -1.2 & $-5.3,2.8$ & 0.56 & -0.5 & $-2.2,1.2$ & 0.54 \\
\hline Depression (past two weeks) & -1.3 & $-4.5,1.9$ & 0.43 & -1.5 & $-5.4,2.5$ & 0.47 \\
\hline Hopelessness (past two weeks) & -1.6 & $-4.4,1.2$ & 0.27 & -0.6 & $-3.7,2.4$ & 0.69 \\
\hline Global social support score & -0.2 & $-1.7,1.2$ & 0.75 & -0.6 & $-2.2,0.9$ & 0.42 \\
\hline \multicolumn{7}{|c|}{$\begin{array}{l}\text { Base Outcome: SI/SA= Suicidal Ideation \& Suicide Attempt } \\
\text { Abbreviations: } \mathbf{A C E}=\text { adverse childhood experiences; } \mathbf{C I}=\text { confidence interval; } \mathbf{L E C}=\text { life events checklist; } \mathbf{O D}=\text { overdose; } \mathbf{S A}=\text { suicide attempt; } \\
\mathbf{S I B}=\text { self-injurious behavior; } \mathbf{S I}=\text { suicidal ideation;SI/SA/OD= patients who self-reported any of the following: } 1 \text { ) suicidal ideation, } 2 \text { ) suicidal } \\
\text { ideation and intentional overdose, 3) suicide attempt and unintentional overdose, or unintentional overdose, suicidal ideation, and suicide attempt. }\end{array}$} \\
\hline
\end{tabular}




\section{Chapter 5}

\section{Discussion of Findings and Research Implications}

\subsection{Summary}

The literature review presented in this study is the first to include self-injurious behaviors to evaluate the relationship between suicide, overdose, and OUD. Results from this review provided the foundation for implementation of patient safety screening for overdose and suicide history in addiction treatment facilities. The mPSS is potentially the first screening tool to simultaneously assess suicide risk and overdose in these setting. Additionally, the brevity of the mPSS increases the probability of integration in routine clinical practice. Results from initial implementation of these screening procedures indicates that persistence of non-fatal SIB needs continued assessment over the course of the lifespan, paying attention to how these behaviors may evolve in adulthood. Furthermore, traumatic childhood events and past year stressful experiences are important in SIB assessment. Future research needs to address comprehensive discharge planning and risk management for both suicide and overdose. This includes provision of global social support, acute and long-term crisis management, and trauma informed care.

\subsection{Strengths and Limitations}

\subsubsection{Strengths}

Strengths of this study are that it includes the first systematic review to focus on selfinjurious behavior, overdose, and suicidal behaviors. While several systematic reviews have studied these relationships to varying degrees in the past, this review is the first to examine literature that reports on overdose and SIB simultaneously. Additionally, this review examines

precursory behaviors to suicide and overdose among individuals with a specific focus on OUD. This literature review was further used to inform a feasibility and acceptability study of the first 
standardized tool for universal suicide and overdose screening for patients receiving addiction treatment services. Although, suicide screening and assessment is usually a part of standard mental healthcare procedures, behavioral health practices do not consistently evaluate additional self-harm and overdose behaviors. This study provided a standardized tool that could be integrated into clinical framework and address these aspects with one instrument. Finally, through examination of subjects who screened positive on the MPSS, patients could be separated into three distinct clinical groups: 1) unintentional overdose only, 2) suicidal ideation/suicide attempt, and 3) unintentional overdose/suicidal ideation/suicide attempt. This allowed for preliminary investigation of clinical and behavioral characteristics that may provide more insight for distinguish intentional and unintentional overdose. Elucidating these factors would ultimately have implications for treatment planning and risk reduction.

\subsubsection{Limitations}

This study has some potential limitations. In conducting the systematic review, the majority of studies was cross-sectional. Thus, temporal conclusions could not be drawn that linked onset of OUD with SIB, suicidal ideation, and/or attempted suicide. A large portion of the available data relied on self-report measures for attempted suicide history, overdose history, and some clinical data. Therefore, recall bias was a predominant research limitation. Furthermore, there is limited generalizability of the studies conducted among inpatient or outpatient treatment samples. Unknown is whether treatment seeking individuals have more functional impairments or experience more symptom severity than non-treatment seeking individuals.

Additionally, several limitations are associated with the studies conducted among patients receiving addiction treatment services. Recruiting subjects from an inpatient population may limit whether these results can be generalized to patients seeking treatment services in other 
settings. The majority of the subjects recruited for this study was predominantly white and nonHispanic, which may not generalize to other ethnic and demographic groups. All assessments were administered by members of the study team. However, in order to integrate these tools into clinical practice, members of the clinical team would need to administer the screening to patients or have patients self-administer the tool. This may require more individualized time from the staff, and increased cooperation from patients that could increase the burden on both patients and staff. To avoid the confounding effects of acute intoxication on our results, patients were not interviewed for at least 24-hours after admission. However, this cautionary measure may not be practical in all treatment settings. Likewise, despite specification of overdose as "decreased respiratory depression," subjects reported non-opioid related overdoses. Lifetime suicidal ideation, overdose(s), and suicide attempt(s) were retrospectively self-reported, and thus recall bias may be a potential limitation.

\subsection{Conclusions and Implications}

The relationship between self-injurious behaviors, suicidal ideation, suicide planning, and suicide attempt has been investigated to varying degrees throughout the published literature. Individuals with these behaviors demonstrate decreased social function, psychiatric decline, and more severe substance. This finding provides crucial information for potential clinical intervention, treatment, and crisis management. However, there is still a lack of prospective longitudinal data that provide adequate temporal information between onset of OUD, SIB and/or suicide outcomes. Previous literature has found that patients with OUD at highest risk of attempted suicide and SIB tended to have more severe psychiatric profiles, be female, have increased rates of poly-drug use, and experienced significant life stressors and childhood maltreatment. In this study, $60 \%$ of patients screened positive on the mPSS, with $86 \%$ of subjects 
screening positive on more than one factor. Further assessment found that approximately $20 \%$ of subjects experienced an unintentional overdose with no suicidal ideation, and $30 \%$ of the sample experienced all three events: an unintentional overdose, suicidal ideation, and at least one suicide attempt during their lifetime. Results from this study appear to be the most compelling between the unintentional overdose and suicidal ideation/suicide attempt group. Differences most commonly related to a history of lifetime substance use, substances used in the 24-hours prior to overdose, physical or emotional abuse during childhood, and frequency of lifetime SIB.

Overall, this study highlights the importance of addressing the contribution of history of self-injury, types of substances used the day of overdose, and ACEs for both overdose and suicide among patients with OUD. These patients may engage in a range of high-risk behaviors that further complicate developing effective risk reduction strategies and effective discharge planning. While there are distinctive characteristics between individuals that have unintentional overdoses only and individuals with suicidal ideation/suicide attempts without history of overdose; patients with a history of all three events may complicate the overall picture and require more complex clinical interventions. Despite, differences in substance use that distinguish intentional from unintentional overdoses, future research needs to focus on systematically addressing substance typologies in overdose. However, additional research is needed to determine whether screening improves the provision of services and reduces future associated self-injurious behavior. In general, the greater the burden of risk factors, the higher the risk for potential self-injury and attempted suicide. 


\section{References}

1. Ahmedani BK, Simon GE, Stewart C, et al. Health care contacts in the year before suicide death. Journal of general internal medicine. 2014;29(6):870-877.

2. Ashrafioun, L., Bishop, T. M., Conner, K. R., Pigeon, W. R., 2017. Frequency of prescription opioid misuse and suicidal ideation, planning, and attempts. J. Psychiatr. Res. 92, 1-7.

3. Bajaj P, Borreani E, Ghosh P, Methuen C, Patel Crawford MJ (2008). Screening for suicidal thoughts in primary care: the views of patients and general practitioners. Mental Health Family Medicine 5, 229-235.

4. Bargagli AM, Hickman M, Davoli M, et al; COSMO European Group. Drug-related mortality and its impact on adult mortality in eight European countries. Eur J Public Health. 2006;16(2):198-202.

5. Baykara, S., Alban, K., 2018. The relationship between self-harming behavior, suicide attempt history and defense mechanisms in patients with opioid-use disorder. Dusunen Adam-J. Psychiatry Neurol. Sci. 31(3), 265-273.

6. Beck, A. T., Kovacs, M., \& Weissman, A. (1979). Assessment of suicidal intention: The Scale for Suicide Ideation. Journal of Consulting and Clinical Psychology, 47(2), 343352. https://doi.org/10.1037/0022-006X.47.2.343

7. Bennett, A. S., Elliott, L., Golub, A., Wolfson-Stofko, B., \& Guarino, H. (2017). Opioidinvolved overdose among male Afghanistan/Iraq-era US military veterans: a multidimensional perspective. Substance use \& misuse, 52(13), 1701-1711.

8. Best, D., Gossop, M., Man, L., Finch, E., Greenwood, J., Strang, J., 2000. Accidental and deliberate overdose among opiate addicts in methadone maintenance treatment: are deliberate overdoses systematically different? Drug Alcohol Rev. 19(2), 213-216.

9. Biddle L, Cooper J, Owen-Smith A, Klineberg E, Bennewith O, Hawton K, Kapur N, Donovan J, Gunnel D (2013). Qualitative interviewing with vulnerable populations: Individuals' experiences of participating insuicide and self-harm-based research. Journal of AffectiveDisorders 145, 356-362.

10. Biggerstaff $\mathrm{D}$, \& Thompson, A. R. Interpretative phenomenological analysis (IPA): A qualitative methodology of choice in healthcare research. Qualitative research in psychology. 2008;5(3):10.

11. Bogdanowicz, K. M., Stewart, R., Chang, C., Downs, J., Khondoker, M., Shetty, H., Strang, J., Hayes, R. D., 2016. Identifying mortality risks in patients with opioid use disorder using brief screening assessment: Secondary mental health clinical records analysis. Drug Alcohol Depend. 164, 82-88. 
12. Bohnert, A. S., \& Ilgen, M. A. (2019). Understanding links among opioid use, overdose, and suicide. New England journal of medicine, 380(1), 71-79.

13. Bohnert AS, Roeder K, Ilgen MA. Unintentional overdose and suicide among substance users: a review of overlap and risk factors. Drug and alcohol dependence. 2010;110(3):183192.

14. Bohnert, A. S., Valenstein, M., Bair, M. J., Gonoczy, D., McCarthy, J. F., Ilgen, M. A., Blow, F. C., 2011. Association between opioid prescribing patterns and opioid overdoserelated deaths. JAMA. 305(13), 1315-21.

15. Bohnert, K. M., Ilgen, M. A., Louzon, S., McCarthy, J. F., Katz, I. R., 2017. Substance use disorders and the risk of suicide mortality among men and women in the US Veterans Health Administration. Addiction 112(7), 1193-1201.

16. Borges, G., Walters, E. E., Kessler, R. C., 2000. Associations of substance use, abuse, and dependence with subsequent suicidal behavior. Am. J. Epidemiol. 151(8), 781-789.

17. Boudreaux ED, Jaques ML, Brady KM, Matson A, Allen MH. The patient safety screener: validation of a brief suicide risk screener for emergency department settings. Archives of suicide research: official journal of the International Academy for Suicide Research. 2016;19(2):151-160.

18. Boudreaux ED, Miller I, Goldstein AB, et al. The Emergency Department Safety Assessment and Follow-up Evaluation (ED-SAFE): method and design considerations. Contemporary clinical trials. 2013;36(1):14-24.

19. Brådvik, L., Frank, A., Hulenvik, P., Medvedeo, A., Berglund, M., 2007. Heroin addicts reporting previous heroin overdoses also report suicide attempts. Suicide Life Threat. Behav. 37(4), 475-481.

20. Briere, J., Gil, E., 1998. Self-mutilation in clinical and general population samples: prevalence, correlates, and functions. Am. J. Orthopsychiatry 68(4), 609-620.

21. Cavanagh, J. T., Carson, A. J., Sharpe, M., Lawrie, S. M., 2003. Psychological autopsy studies of suicide: a systematic review. Psychol. Med. 33(3), 395-405.

22. Cella, D., Riley, W., Stone, A. A., Rothrock, N., Reeve, B. B., Yount, S., Amtmann, D., D., B., Choi, S., Cook, K. F., et al. (2010). The Patient Reported Outcomes Measurement Information System (PROMIS) developed and tested its first wave of adult selfreported health outcome item banks: 2005-2008. Journal of Clinical Epidemiology 63, 1179-1194.

23. Centers for Disease Control and Prevention, National Center for Injury Prevention and Control. (2017). Web-based Injury Statistics Query and Reporting System (WISQARS) [online]. Retrieved 2020-2-63 www.cdc.gov/injury/wisqars 
24. Cheek, S. M., Nestor, B. A., Liu, R. T., 2016. Substance use and suicidality: specificity of substance use by injection to suicide attempts in a nationally representative sample of adults with major depression. Depress. Anxiety 33(6), 541-548.

25. Chen, V. C., Cheng, A. T., Tan, H. K., Chen, C. Y., Chen, T. H., Stewart, R., Prince, M., 2009. A community-based study of case fatality proportion among those who carry out suicide acts. Soc. Psychiatry Psychiatr. Epidemiol. 44(12), 1005-11.

26. Chen, V. C. H., Lin, T. Y., Lee, C. T. C., Lai, T. J., Chen, H., Ferri, C. P., Gossop, M., 2010. Suicide attempts prior to starting methadone maintenance treatment in Taiwan. Drug Alcohol Depend. 109(1-3), 139-143.

27. Colledge, S., Larney, S., Peacock, A., Leung, J., Hickman, M., Grebely, J., Farrell, M., Degenhardt, L., 2019. Depression, post-traumatic stress disorder, suicidality and self-harm among people who inject drugs: a systematic review and meta-analysis. Drug Alcohol Depend. 107793.

28. Commission TJ. Detecting and Treating Suicide Ideation in All Settings. In:2016.

29. Conner KR, Beautrais AL, Conwell Y. Risk factors for suicide and medically serious suicide attempts among alcoholics: analyses of Canterbury suicide project data. J Stud Alcohol Drugs. 2003;64:551-554.

30. Conner, K. R., Britton, P. C., Sworts, L. M., Joiner Jr, T. E., 2007. Suicide attempts among individuals with opiate dependence: The critical role of belonging. Addict. Behav. 32(7), 1395-1404.

31. Connery, H. S., Taghian, N., Kim, J., Griffin, M., Rockett, I. R., Weiss, R. D., \& McHugh, R. K. (2019). Suicidal motivations reported by opioid overdose survivors: A cross-sectional study of adults with opioid use disorder. Drug and alcohol dependence, 205, 107612.

32. Cooper, J., Kapur, N., Webb, R., Lawlor, M., Guthrie, E., Mackway-Jones, K., Appleby, L., 2005. Suicide after deliberate self-harm: a 4-year cohort study. Am. J. Psychiatry 162(2), 297-303.

33. CSAT, C.f.S.A.T., Addressing Suicidal thoughts and Behaviors in Substance Abuse Treatment. 2009, SAMHSA: Rockville, MD.

34. Crosby, A. E., Han, B., Ortega, L. A. G., Parks, S. E., \& Gfroerer, J. (2011, October 21). Suicidal thoughts and behaviors among adults aged $\geq 18$ years-United States, 20082009. Morbidity and Mortality Weekly Report Surveillance Summaries, 60(SS13), 1-22. Retrieved from https://www.cdc.gov/mmwr/preview/mmwrhtml/ss6013a1.htm 
35. Cunningham, R. M., Walton, M. A., \& Carter, P. M. (2018). The major causes of death in children and adolescents in the United States. New England Journal of Medicine, 379(25), 2468-2475.

36. Cumming S, Covic T, Murrell E. Deliberate self-harm: have we scratched the surface? Behav Change 2006;23:186-99.

37. Dai, Z., Abate, M. A., Smith, G. S., Kraner, J. C., \& Mock, A. R., 2019. Fentanyl and fentanyl-analog involvement in drug-related deaths. Drug Alcohol Depend. 196, 1-8.

38. Darke, S., Campbell, G., Popple, G., 2012. Self-harm and attempted suicide among therapeutic community admissions. Drug Alcohol Rev. 31(4), 523-528.

39. Darke S, Degenhardt L, Mattick R. Mortality amongst illicit drug users: epidemiology, causes and intervention. Cambridge: Cambridge University Press, 2007.

40. Darke S, Kaye S. Attempted suicide among injecting and non-injecting cocaine users in Sydney, Australia. J Urban Health 2004; 81:505-15.

41. Darke, S., Mills, K. L., Ross, J., \& Teesson, M. (2011). Rates and correlates of mortality amongst heroin users: findings from the Australian Treatment Outcome Study (ATOS), 2001-2009. Drug and alcohol dependence, 115(3), 190-195.

42. Darke S, Ross J. Suicide among heroin users: rates, risk factors and methods. Addiction (Abingdon, England). 2002;97(11):1383-1394.

43. Darke, S., Ross, J., 2001. The relationship between suicide and heroin overdose among methadone maintenance patients in Sydney, Australia. Addiction 96(10), 1443-1453.

44. Darke, S., Ross, J., Lynskey, M., Teesson, M., 2004. Attempted suicide among entrants to three treatment modalities for heroin dependence in the Australian Treatment Outcome Study (ATOS): prevalence and risk factors. Drug Alcohol Depend. 73(1), 1-10.

45. Darke, S., Ross, J., Marel, C., Mills, K. L., Slade, T., Burns, L., Teesson, M., 2015. Patterns and correlates of attempted suicide amongst heroin users: 11-year follow-up of the Australian treatment outcome study cohort. Psychiatry Res. 227(2-3), 166-170.

46. Darke S, Ross J, Williamson A, Mills KL, Havard A, Teesson M. Patterns and correlates of attempted suicide by heroin users over a three-year period: findings from the Australian Treatment Outcome Study. Drug Alcohol Depend 2007; 87:146-52.

47. Darke, S., Torok, M., Kaye, S., Ross, J., 2010. Attempted suicide, self-harm, and violent victimization among regular illicit drug users. Suicide Life Threat. Behav. 40(6), 587-596.

48. Davidson JR, et al. The association of sexual assault and attempted suicide within the community. Arch Gen Psychiatry. 1996;53:550-555. 
49. de los Cobos, J. P., Trujols, J., Ribalta, E., Pinet, C., 2009. A typology of heroin-dependent patients based on their history of self-injurious behaviours. Psychiatry Res. 167(1-2), 169177.

50. Degenhardt, L., Buceelo, C., Mathers, B., Briegleb, C., Ali, H., Hickman, M., McLaren, J., 2011. Mortality among regular or dependent users of heroin and other opioids: a systematic review and meta-analysis of cohort studies. Addiction 106, 32-51.

51. Degenhardt L, Larney S, Randall D, Burns L, Hall W. Causes of death in a cohort treated for opioid dependence between 1985 and 2005. Addiction. 2014;109(1):90-99.

52. Derefinko, K. J., García, F. I. S., Talley, K. M., Bursac, Z., Johnson, K. C., Murphy, J. G., ... \& Sumrok, D. D. (2019). Adverse childhood experiences predict opioid relapse during treatment among rural adults. Addictive behaviors, 96, 171-174.

53. D’Onofrio G, Chawarski MC, O’Connor PG, et al. Emergency department-initiated buprenorphine for opioid dependence with continuation in primary care: outcomes during and after intervention. J Gen Intern Med. 2017;32(6):660-666

54. Dowell D, Arias E, Kochanek K, et al. Contribution of Opioid-Involved Poisoning to the Change in Life Expectancy in the United States, 2000-2015. JAMA. 2017;318(11):10651067. doi:10.1001/jama.2017.9308

55. Dragisic, T., Dickov, A., Dickov, V., Mijatovic, V., 2015. Drug addiction as risk for suicide attempts. Mater. Sociomed. 27(3), 188.

56. Evren C, Evren B. Self-mutilation in substance-dependent patients and relationship with childhood abuse and neglect, alexithymia and temperament and character dimensions ofpersonality. Drug Alcohol Depend 2005;80:15-22.

57. Favazza AR. The coming of age of self-mutilation. J Nerv Ment Dis 1998;186:259-68.

58. Foster T, Gillespie K, McClelland R. Mental disorders and suicide in Northern Ireland. The British journal of psychiatry : the journal of mental science. 1997;170:447-452.

59. Frazier W, Cochran G, Lo-Ciganic WH, et al. Medication-assisted treatment and opioid use before and after overdose in Pennsylvania Medicaid.JAMA. 2017;318(8):750-752.

60. Gairin I, Allan House, and David Owens. Attendance at the accident and emergency department in the year before suicide: retrospective study. The British Journal of Psychiatry 2003;1(183):6.

61. Ghaffari-Nejad, A., Mehdizadeh-Zareanari, A., Pouya, F., Mashroteh, M., 2012. Suicide in narcotic drugs dependents. Addict. Health 4(1-2), 43. 
62. Gibson A, Randall D, Degenhardt L. The increasing mortality burden of liver disease among opioid-dependent people: cohort study. Addiction. 2011;106(12):2186-2192.

63. Gilmore, A. K., Hahn, C. K., Jaffe, A. E., Walsh, K., Moreland, A. D., Ward-Ciesielski, E. F., 2018. Suicidal ideation among adults with a recent sexual assault: prescription opioid use and prior sexual assault. Addict. Behav. 85, 120-124.

64. Håkansson, A. C., Brådvik, L., Schlyter, F., Berglund, M., 2010. Factors associated with the history of attempted suicide. Crisis 31(1), 12-21.

65. Håkansson, A. C., Schlyter, F., Berglund, M., 2008. Factors associated with history of nonfatal overdose among opioid users in the Swedish criminal justice system. Drug Alcohol Depend. 94(1-3), 48-55.

66. Harned, M. S., Najavits, L. M., Weiss, R. D., 2006. Self-harm and suicidal behavior in women with comorbid PTSD and substance dependence. Am. J. Addict. 15(5), 392-395.

67. Harris, E. C., Barraclough, B., 1997. Suicide as an outcome for mental disorders. A metaanalysis. Br. J. Psychiatry 170(3), 205-228.

68. Hasegawa, K., Brown, D.F.M., Tsugawa, Y., Camargo, C.A., 2014. Epidemiology of emergency department visits for opioid overdose: a population-based study. Mayo Clin. Proc. 89, 462-471.

69. Havens, J. R., Strathdee, S. A., Fuller, C. M., Ikeda, R., Friedman, S. R., Des Jarlais, D. C., Morse, P. S., Bailey, S., Kerndt, P., Garfein, R. S., Collaborative Injection Drug User Study Group, 2004. Correlates of attempted suicide among young injection drug users in a multisite cohort. Drug Alcohol Depend. 75(3), 261-269.

70. Hawton, K., Saunders, K. E., O'Connor, R. C., 2012. Self-harm and suicide in adolescents. Lancet 379(9834), 2373-2382.

71. Heale, P., Dietze, P., Fry, C., 2003. Intentional overdose among heroin overdose survivors. J. Urban Health 80(2), 230-237.

72. Hedegaard, H., Warner, M., Miniño, A. M., 2018. Drug overdose deaths in the United States, 1999-2017. NCHS Data Brief, no 294. National Center for Health Statistics, Hyattsville, MD.

73. Icick, R., Karsinti, E., Lépine, J. P., Bloch, V., Brousse, G., Bellivier, F., Vorspan, F., 2017. Serious suicide attempts in outpatients with multiple substance use disorders. Drug Alcohol Depend. 181, 63-70.

74. Ilgen, M. A., Chermack, S. T., Murray, R., Walton, M. A., Barry, K. L., Wojnar, M., Blow, F. C., 2009. The association between partner and non-partner aggression and suicidal 
ideation in patients seeking substance use disorder treatment. Addict. Behav. 34(2), 180186.

75. International Society for the Study of Self-Injury, 2007. Definition of nonsuicidal selfinjury. http://www.itriples.org/isss-aboutself-i.html. (accessed 12.12.19).

76. Jin, H., Atkinson, J. H., Duarte, N. A., Yu, X., Shi, C., Riggs, P. K., Li, J., Gupta, S., Wolfson, T., Knight, A. F., Franklin, D., Letendre, S., Wu, Z., Grant, I., Heaton, R. K., HNRC China Collaboration Group, 2013. Risks and predictors of current suicidality in HIV-infected heroin users in treatment in Yunnan, China: a controlled study. J. Acquir. Immune Defic. Syndr. 62(3), 311.

77. Kalyoncu, A., Mırsal, H., Pektaş, Ö., Tan, D., Beyazyürek, M., 2007. Heroin-dependent patients attempting and not attempting suicide: a comparison. Acta Neuropsychiatr. 19(5), 297-303.

78. Kazour, F., Soufia, M., Rohayem, J., Richa, S., 2016. Suicide risk of heroin dependent subjects in Lebanon. Community Ment. Health J. 52(5), 589-596.

79. Kelty, E., Hulse, G. G., 2017. Fatal and non-fatal opioid overdose in opioid dependent patients treated with methadone, buprenorphine, or implant naltrexone. Int. J. Drug Policy $46,54-60$.

80. Kerr, T., Small, W., Hyshka, E., Maher, L., \& Shannon, K. (2013). 'It's more about the heroin': injection drug users' response to an overdose warning campaign in a Canadian setting. Addiction, 108(7), 1270-1276.

81. King CA, O'Mara RM, Hayward CN, Cunningham RM. Adolescent suicide risk screening in the emergency department. Academic emergency medicine : official journal of the Society for Academic Emergency Medicine. 2009;16(11):1234-1241.

82. King R, Nurcombe B, Bickman L, Hides L, Reid W (2003). Telephone counselling for adolescent suicide prevention: changes in suicidality and mental state from beginning to end of a counselling session. Suicide \& Life-Threatening Behaviour 33, 400-411.

83. Kuramoto, S. J., Chilcoat, H. D., Ko, J., Martins, S. S., 2012. Suicidal ideation and suicide attempt across stages of nonmedical prescription opioid use and presence of prescription opioid disorders among US adults. J. Stud. Alcohol Drugs 73(2), 178-184.

84. Lander, L. R., Zheng, W., Hustead, J. D., Mahoney III, J. J., Berry, J. H., Marshalek, P., Winstanley, E. L., 2020. Long-term treatment retention in West Virginia's comprehensive opioid addiction treatment (COAT) program. J. Neurol. Sci. 411.

85. Lang M, Uttaro T, Caine E, Carpinello S, Felton C (2010). Implementing routine suicide risk screening for psychiatric outpatients with serious mental disorders: II. Quantitative results. Archives of Suicide Research 13, 169-177. 
86. Larney S, Randall D, Gibson A, Degenhardt L. The contributions of viral hepatitis and alcohol to liver-related deaths in opioid-dependent people. Drug Alcohol Depend. 2013;131(3):252-257.

87. Larochelle, M. R., Liebschutz, J. M., Zhang, F., Ross-Degnan, D., Wharam, J. F., 2016. Opioid prescribing after nonfatal overdose and association with repeated overdose: a cohort study. Ann. Intern. Med. 164, 1-9.

88. Lavania, S., Ram, D., Praharaj, S. K., Khan, A. H., Pattojoshi, A., 2012. Deliberate selfharm in nondepressed substance-dependent patients. J. Addict. Med. 6(4), 247-252.

89. Legerski, J. P., \& Bunnell, S. L. (2010). The risks, benefits, and ethics of trauma-focused research participation. Ethics \& Behavior, 20(6), 429-442.

90. Lish JD, Zimmerman M, Farber NJ, Lush DT, Kuzma MA, Plescia G. Suicide screening in primary care setting at a Veterans Affairs Medical Center. Psychosomatics. 1996;37(5):413-424.

91. Maloney, E., Degenhardt, L., Darke, S., Mattick, R. P., Nelson, E., 2007. Suicidal behaviour and associated risk factors among opioid-dependent individuals: A case-control study. Addiction 102(12), 1933-1941.

92. Maloney, E., Degenhardt, L., Darke, S., Nelson, E. C., 2010. Investigating the cooccurrence of self-mutilation and suicide attempts among opioid-dependent individuals. Suicide Life Threat. Behav. 40(1), 50-62.

93. Manyema, M., Norris, S. A., \& Richter, L. M. (2018). Stress begets stress: the association of adverse childhood experiences with psychological distress in the presence of adult life stress. BMC public health, 18(1), 835.

94. Marchand, K., Palis, H., Fikowski, J., Harrison, S., Spittal, P., Schechter, M. T., OviedoJoekes, E., 2017. The role of gender in suicidal ideation among long-term opioid users. Can. J. Psychiatry 62(7), 465-472.

95. Maremmani, I., Pani, P. P., Canoniero, S., Pacini, M., Perugi, G., Rihmer, Z., Akiskal, H. S., 2007. Is the bipolar spectrum the psychopathological substrate of suicidality in heroin addicts? Psychopathology 40(5), 269-277.

96. Masferrer, L., Garre-Olmo, J., Caparros, B., 2016. Risk of suicide: Its occurrence and related variables among bereaved substance users. J. Subst. Use 21(2), 191-197.

97. Miller, P. G. (2009). Safe using messages may not be enough to promote behaviour change amongst injecting drug users who are ambivalent or indifferent towards death. Harm reduction journal, 6(1), 18. 
98. Naeger S, Mutter R, Ali MM, Mark T, Hughey L. Post-discharge treatment engagement among patients with an opioid-use disorder.J Subst Abuse Treat. 2016;69:64-71.

99. Neale, J., 2000. Suicidal intent in non-fatal illicit drug overdose. Addiction 95(1), 85-93.

100. Nock, M. K., Holmberg, E. B., Photos, V. I., \& Michel, B. D. (2007). The Self-Injurious Thoughts and Behaviors Interview: Development, reliability, and validity in an adolescent sample. Psychological Assessment, 19, 309-317.

101. Norman, S. B., Tate, S. R., Anderson, K. G., \& Brown, S. A. (2007). Do trauma history and PTSD symptoms influence addiction relapse context?. Drug and alcohol dependence, 90(1), 89-96.

102. O'Connor, R. C., Nock, M. K., 2014. The psychology of suicidal behavior. Lancet Psychiatry 1(1), 73-85.

103. O'Connor, R. C., 2011. The integrated motivational-volitional model of suicidal behavior. Crisis 32(6), 295-8.

104. Olfson, M., Crystal, S., Wall, M., Wang, S., Liu, S., Blanco, C. "Causes of death after nonfatal opioid overdose." JAMA psychiatry 75, no. 8 (2018): 820-827.

105. Olfson M, Wall M, Wang S, Crystal S, Gerhard T, Blanco C. Suicide following deliberate self-harm. Am J Psychiatry. 2017;174(8):765-774

106. Olsson, M. O., Bradvik, L., Öjehagen, A., Hakansson, A., 2016. Risk factors for unnatural death: Fatal accidental intoxication, undetermined intent and suicide: Register follow-up in a criminal justice population with substance use problems. Drug Alcohol Depend. 162, 176181.

107. Omerov P, Steineck G, Dyregrov K, Runeson B and Nyberg U (2013). The ethics of doing nothing. Suicide-bereavement and research: ethical and methodological considerations. Psychological Medicine. Published online: 19 July 2013. doi: $10.1017 / \mathrm{S} 0033291714001275$.

108. Osman, A; Bagge, CL; Gutierrez, PM; Konick, LC; Kopper, BA; Barrios, FX (December 2001). "The Suicidal Behaviors Questionnaire-Revised (SBQ-R): validation with clinical and nonclinical samples". Assessment. 8 (4): 443-54. doi:10.1177/107319110100800409

109. Oqueno M. Opioid use disorders and suicide: a hidden tragedy. National Institute on Drug Abuse Blog.https://www.drugabuse.gov/about-nida/noras-blog/2017/04/opioid-usedisorders-suicide-hidden-tragedy-guest-blog. Published April 20,2017.

110. Oquendo, M. A., \& Volkow, N. D. (2018). Suicide: a silent contributor to opioid-overdose deaths. New England journal of medicine, 378(17), 1567-1569. 
111. Oyefeso, A., Brown, S., Chiang, Y., Clancy, C., 2008. Self-injurious behaviour, traumatic life events and alexithymia among treatment-seeking opiate addicts: Prevalence, pattern and correlates. Drug Alcohol Depend. 98(3), 227-234.

112. Pan, C. H., Jhong, J. R., Tsai, S. Y., Lin, S. K., Chen, C. C., Kuo, C. J., 2014. Excessive suicide mortality and risk factors for suicide among patients with heroin dependence. Drug Alcohol Depend. 145, 224-230.

113. Park, S., Lee, Y., Youn, T., Kim, B. S., Park, J. I., Kim, H., Lee, H. C., Hong, J. P., 2018. Association between level of suicide risk, characteristics of suicide attempts, and mental disorders among suicide attempters. BMC Public Health 18(1), 477.

114. Penney, A., Mazmanian, D., Jamieson, J., Black, N., 2012. Factors associated with recent suicide attempts in clients presenting for addiction treatment. Int. J. Ment. Health Addiction 10(1), 132-140.

115. Pierce M, Bird SM, Hickman M, Millar T. National record linkage study of mortality for a large cohort of opioid users ascertained by drug treatment or criminal justice sources in England, 2005-2009. Drug Alcohol Depend. 2015;146:17-23.

116. Pilowsky, D. J., Keyes, K. M., Geier, T. J., Grant, B. F., \& Hasin, D. S. (2013). Stressful life events and relapse among formerly alcohol dependent adults. Social work in mental health, 11(2), 184-197.

117. Plener, P. L., Schumacher, T. S., Munz, L. M., Groschwitz, R. C., 2015. The longitudinal course of non-suicidal self-injury and deliberate self-harm: a systematic review of the literature. Borderline Personal. Disord. Emot. Dysregul. 2(1), 2.

118. Pouget, E. R., Bennett, A. S., Elliott, L., Wolfson-Stofko, B., Almeñana, R., Britton, P. C., \& Rosenblum, A. (2017). Development of an opioid-related overdose risk behavior scale (ORBS). Substance abuse, 38(3), 239-244.

119. Randall D, Degenhardt L, Vajdic CM, et al. Increasing cancer mortality among opioiddependent persons in Australia: a new public health challenge for a disadvantaged population. Aust N Z J Public Health. 2011;35(3):220-225.

120. Range LM, Knott EC. Twenty suicide assessment instruments: evaluation and recommendations. Death studies. 1997;21(1):25-58.

121. Ravndal, E., Vaglum, P., 1999. Overdoses and suicide attempts: different relations to psychopathology and substance abuse? A 5-year prospective study of drug abusers. Eur. Addict. Res. 5(2), 63-70.

122. Risser, D., Honigschnabl, S., Stichenwith, M., Pfudl, S., Sebald, D., Kaff, A., Bauer, G., 2001. Mortality of opiate users in Vienna, Austria. Drug Alcohol Depend. 64, 251-256. 
123. Rockett, I. R., \& Caine, E. D. (2015). Self-injury is the eighth leading cause of death in the United States: it is time to pay attention. JAMA psychiatry, 72(11), 1069-1070.

124. Ross, J., Teesson, M., Darke, S., Lynskey, M., Ali, R., Ritter, A., Cooke, R., 2005. The characteristics of heroin users entering treatment: findings from the Australian treatment outcome study (ATOS). Drug Alcohol Rev. 24(5), 411-418.

125. Rossow, I., Lauritzen, G., 1999. Balancing on the edge of death: suicide attempts and lifethreatening overdoses among drug addicts. Addiction 94(2), 209-219.

126. Roy A. Characteristics of cocaine dependent patients who attempt suicide. Arch Suicide Res. 2009;13:46-51.

127. Roy, A., 2002. Characteristics of opiate dependent patients who attempt suicide. J. Clin. Psychiatry 63(5), 403-407.

128. Roy, A., 2010. Risk factors for attempting suicide in heroin addicts. Suicide Life Threat. Behav. 40(4), 416-420.

129. Roy A, Janal. MN. Risk factors for suicide among alcohol-dependent patients. Arch Suicide Res. 2007;11:211-217.

130. Schalinski, I., Teicher, M. H., Nischk, D., Hinderer, E., Müller, O., \& Rockstroh, B. (2016). Type and timing of adverse childhood experiences differentially affect severity of PTSD, dissociative and depressive symptoms in adult inpatients. BMC Psychiatry, 16, 295. https://doi.org/10.1186/s12888-016-1004-5

131. Scholl L, Seth P, Kariisa M, Wilson N, Baldwin G. Drug and Opioid-Involved Overdose Deaths-United States, 2013-2017. MMWR Morb Mortal Wkly Rep 2019; 67: 1419-1427. Doi: http://dx.doi.org/10/14485/mmwr.mm675152e1

132. Seth, P., Rudd, R. A., Noonan, R. K., \& Haegerich, T. M. (2018). Quantifying the epidemic of prescription opioid overdose deaths.

133. Shonkoff, J. P., Garner, A. S., Siegel, B. S., Dobbins, M. I., Earls, M. F., McGuinn, L., ... \& Committee on Early Childhood, Adoption, and Dependent Care. (2012). The lifelong effects of early childhood adversity and toxic stress. Pediatrics, 129(1), e232-e246.

134. StataCorp. 2017. Stata Statistical Software: Release 15. College Station, TX: StataCorp LLC.

135. Stein, M. D., Conti, M. T., Kenney, S., Anderson, B. J., Flori, J. N., Risi, M. M., \& Bailey, G.L. (2017). Adverse childhood experience effects on opioid use initiation, injection drug use, and overdose among persons with opioid use disorder. Drug and Alcohol Dependence, 179, 325-329. https://doi.org/10.1016/j.drugalcdep.2017.07.007. 
136. Stone, D. M., Simon, T. R., Fowler, K. A., Kegler, S. R., Yuan, K., Holland, K. M., IveyStephenson, A. Z., \& Crosby, A. E. (2018, June 8). Vital Signs: Trends in suicide rates United States, 1999-2016 and circumstances contributing to suicide - 27 states, 2015. Morbidity and Mortality Weekly Report, 67(22), 617-624. Retrieved from https://www.cdc.gov/mmwr/volumes/67/wr/pdfs/mm6722a1-H.pdf

137. Stoppe G, Sandholzer H, Huppertz C, Duwe H, Staedt J (1999). Family physicians and the risk of suicide in the depressed elderly. Journal of Affective Disorders 54, 193-198.

138. Stoove, M.A., Dietze, P.M., Jolley, D., 2009. Overdose deaths following previous non-fatal heroin overdose: record linkage of ambulance attendance and death registry data. Drug Alcohol Rev. 28, 347-352.

139. Strine, T. W., Dube, S. R., Edwards, V. J., Prehn, A. W., Rasmussen, S., Wagenfeld, M., \& Croft, J. B. (2012). Associations between adverse childhood experiences, psychological distress, and adult alcohol problems. American Journal of Health Behavior, 36(3), 408-423.

140. Substance Abuse and Mental Health Services Administration (2018). The role of adverse childhood experiences in substance abuse and related behavioral health problems. (Retrieved from: https:/www.samhsa.gov/capt/sites/default/files/resources/acesbehavioralhealth-problems.pdf. Accessed 5/3/19).

141. Substance Abuse and Mental Health Services Administration. (2019). Key substance use and mental health indicators in the United States: Results from the 2018 National Survey on Drug Use and Health (HHS Publication No. PEP19-5068, NSDUH Series H-54). Rockville, MD: Center for Behavioral Health Statistics and Quality, Substance Abuse and Mental Health Services Administration. Retrieved from https:/www.samhsa.gov/data/

142. Swendsen, J., Conway, K. P., Degenhardt, L., Glantz, M., Jin, R., Merikangas, K. R., ... \& Kessler, R. C. (2010). Mental disorders as risk factors for substance use, abuse and dependence: results from the 10-year follow-up of the National Comorbidity Survey. Addiction, 105(6), 1117-1128.

143. Tanguturi Y, Bodic M, Taub A, Homel P, Jacob T. Suicide Risk Assessment by Residents: Deficiencies of Documentation. Academic psychiatry: the journal of the American Association of Directors of Psychiatric Residency Training and the Association for Academic Psychiatry. 2017;41(4):513-519.

144. Thompson MP, Kingree JB, Lamis D. Associations of adverse childhood experiences and suicidal behaviors in adulthood in a US nationally representative sample. Child: care, health and development. 2019 Jan;45(1):121-8.

145. Trémeau, F., Darreye, A., Staner, L., Corrêa, H., Weibel, H., Khidichian, F., Macher, J. P., 2008. Suicidality in opioid-dependent subjects. Am. J. Addict. 17(3), 187-194. 
146. Turecki, G., Brent, D. A., 2016. Suicide and suicidal behaviour. Lancet 387(10024), 12271239.

147. Vaismoradi M, Turunen H, Bondas T. Content analysis and thematic analysis: Implications for conducting a qualitative descriptive study. Nursing \& health sciences. 2013;15(3):398405.

148. VanHouten, J. P., Rudd, R. A., Ballesteros, M. F., \& Mack, K. A. (2019). Drug overdose deaths among women aged 30-64 years-United States, 1999-2017. Morbidity and Mortality Weekly Report, 68(1), 1.

149. Vingoe, L., Welch, S., Farrell, M., Strang, J., 1999. Heroin overdose among a treatment sample of injecting drug misusers: accident or suicidal behaviour? J. Subst. Use 4(2), 8891.

150. Voss WD, et al. Preventing addiction related suicide: a pilot study. J Subst Abuse Treat. 2013;44:565-569.

151. Wagstaff C, Williams B. Specific design features of an interpretative phenomenological analysis study. Nurse researcher. 2014;21(3):8-12.

152. Weathers, F. W., Blake, D. D., Schnurr, P. P., Kaloupek, D. G., Marx, B. P., \& Keane, T. M. (2013). The Life Events Checklist for DSM-5 (LEC-5) - Standard. [Measurement instrument]. Available from https://www.ptsd.va.gov/

153. Weiss AJ, Elixhauser A, Barret ML, Steiner CA, Bailer MK, O’Malley L. Opioid-related inpatient stays and emergency department visits by state, 2009-2014; Statistical Brief 219. https://www.hcup-us.ahrq.gov/reports/statbriefs /sb219-Opioid-Hospital-Stays-ED-Visitsby-State .jsp. Published December 2016.

154. Wilcox, H. C., Conner, K. R., Caine, E. D., 2004. Association of alcohol and drug use disorders and completed suicide: an empirical review of cohort studies. Drug Alcohol Depend. 76, S11-S19.

155. Wines Jr, J. D., Saitz, R., Horton, N. J., Lloyd-Travaglini, C., Samet, J. H., 2004. Suicidal behavior, drug use and depressive symptoms after detoxification: a 2-year prospective study. Drug Alcohol Depend. 76, S21-S29.

156. Winstanley, E. L., Lander, L. R., Berry, J. H., Mahoney III, J. J., Zheng, W., Herschler, J., Marshalek, P., Sayres, S., Mason, J., Haut, M. W., 2020. West Virginia's model of buprenorphine expansion: Preliminary results. J. Subst. Abuse Treat. 108, 40-47.

157. WV Health Statistics Center (2017). West Virginia Behavioral Risk Factor Surveillance System Report, 2014. Retrieved on 13 January 2020 from http://www.wvdhhr.org/bph/hsc/pubs/brfss/2014/BRFSS2014.pdf. 
158. Yuodelis-Flores, C., \& Ries, R. K. (2015). Addiction and suicide: a review. The American journal on addictions, 24(2), 98-104. 


\section{Appendices}

\subsection{Supplemental Figure (Chapter 2): Systematic Review Themes}

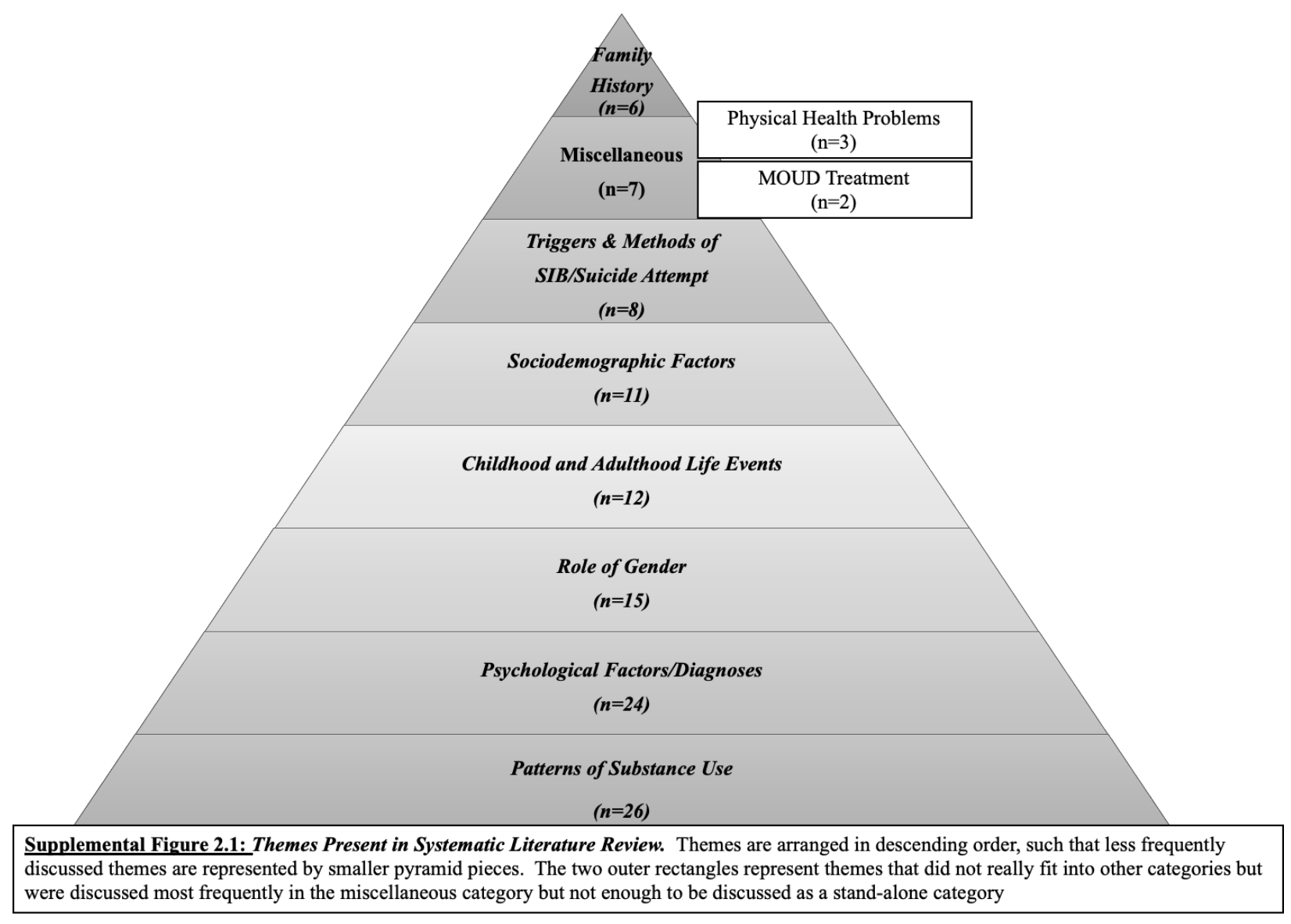


7.2 Supplemental Figure (Chapter 3): Modified Patient Safety Screen

\section{Modified Patient Safety Screener}

1. Over the past 2 weeks, have you felt down, depressed, or hopeless?

Yes No Patient unable to complete Patient refused

2. Over the past 2 weeks, have you have you had thoughts of killing yourself?

Yes No Patient unable to complete Patient refused

3. In your lifetime, have you attempted to kill yourself?

Yes No Patient unable to complete Patient refused

3a. When did his happen?

Within the past 24 hours Within the past month (not including today) In the past 1-6 months

More than 6 months ago Patient unable to complete Patient refused

4. In your lifetime, have you ever overdosed?

Yes No Patient unable to complete Patient refused

4a. When did this happen?

Within the past 24 hours Within the past month (not including today) In the past 1-6 months

More than 6 months ago Patient unable to complete Patient refused 
7.3 Supplemental Figure (Chapter 3)

Visualization of Positive mPSS Screening Responses

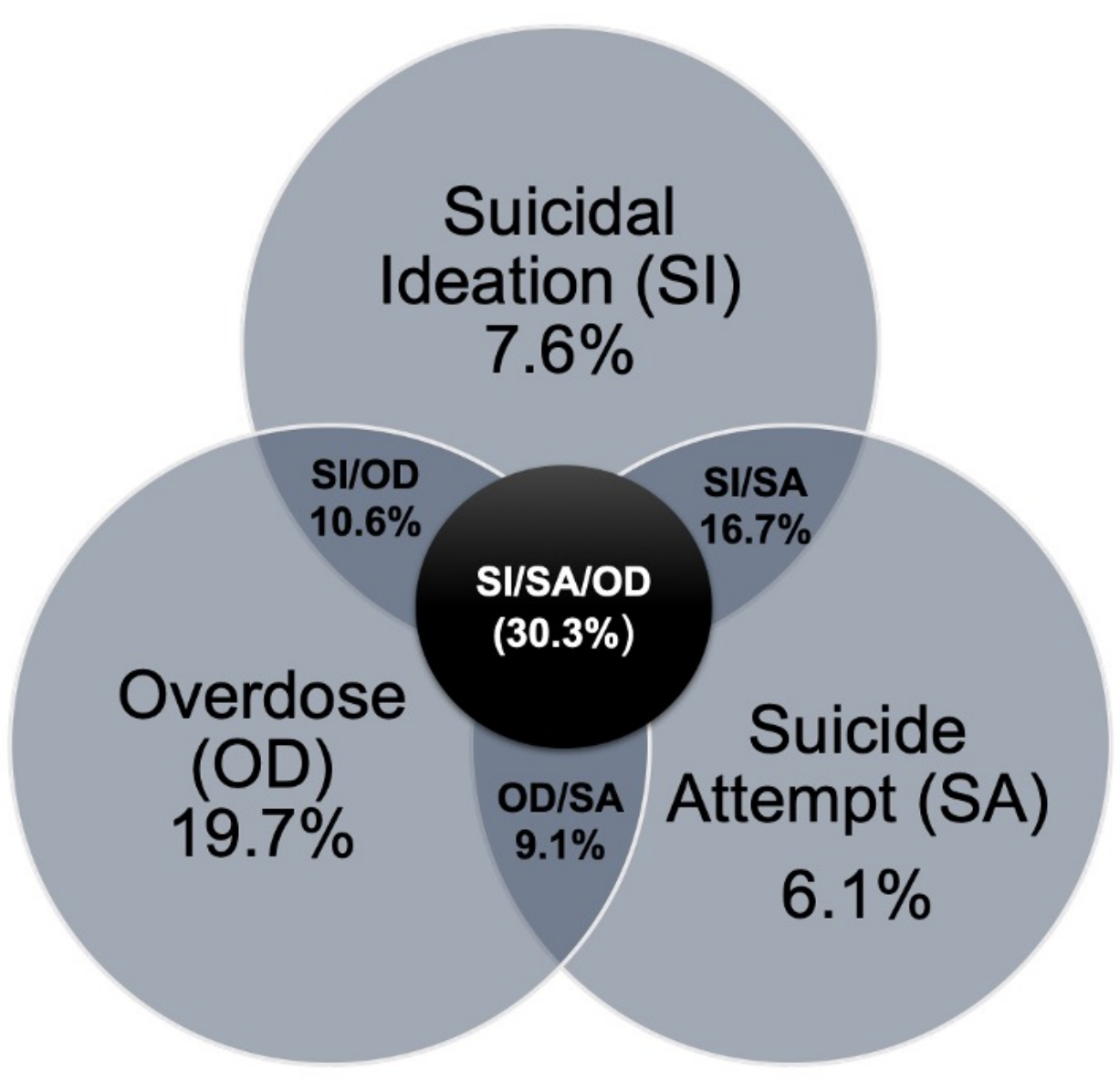




\subsection{Supplemental Table (Chapter 4)}

\begin{tabular}{|c|c|c|c|c|c|}
\hline & $\begin{array}{c}\text { Overall } \\
\text { Sample } \\
(\mathrm{n}=66)\end{array}$ & $\begin{array}{c}\text { Unintentional } \\
\text { OD }^{1} \\
(n=13) \\
\end{array}$ & $\begin{array}{l}\text { SISA }^{2} \\
(n=33)\end{array}$ & $\begin{array}{l}\mathrm{SI} / \mathbf{S A} / \mathbf{O D}^{3} \\
\quad(\mathbf{n}=\mathbf{2 0})\end{array}$ & p-Value \\
\hline \multicolumn{6}{|l|}{ Characteristics } \\
\hline Mean Age (years), (SD) & $36.1(9.5)$ & $36.1(9.3)$ & $37.3(9.6)$ & $34.1(9.7)$ & 0.47 \\
\hline Sex (Male) & 65.2 & 69.2 & 63.6 & 65.0 & 1.00 \\
\hline White & 93.4 & 100.0 & 93.9 & 90.0 & 0.66 \\
\hline Non-Hispanic & 93.4 & 100.0 & 90.9 & 95.0 & 0.81 \\
\hline Employed & 25.8 & 23.1 & 21.2 & 35.0 & 0.57 \\
\hline Education & & & & & 0.68 \\
\hline Less than High School & 19.7 & 23.1 & 24.2 & 10.0 & \\
\hline High School Grad/GED & 50.0 & 46.2 & 51.5 & 50.0 & \\
\hline Some College/College Grad & 30.3 & 30.8 & 24.2 & 40.0 & \\
\hline Lifetime Arrests (Median) & 5 & 3 & 3 & 6 & 0.88 \\
\hline \multicolumn{6}{|l|}{ Lifetime Substance Use (\% Yes) } \\
\hline Tobacco & 98.5 & 100.0 & 97.0 & 100.00 & 1.00 \\
\hline Alcohol & 100.0 & 100.0 & 100.0 & 100.0 & - \\
\hline Cocaine & 84.9 & 100.0 & 72.3 & 95.0 & 0.02 \\
\hline Marijuana & 97.0 & 100.0 & 97.0 & 95.0 & 1.00 \\
\hline Heroin & 87.9 & 92.3 & 81.8 & 95.00 & 0.43 \\
\hline Prescription Opioids & 89.4 & 69.2 & 97.0 & 90.0 & 0.03 \\
\hline Buprenorphine to get high & 39.4 & 38.5 & 39.5 & 40.0 & 1.00 \\
\hline Methamphetamines/Amphetamines & 87.9 & 84.6 & 87.9 & 90.0 & 1.00 \\
\hline Benzodiazepines & 69.7 & 69.2 & 69.7 & 70.0 & 1.00 \\
\hline \multicolumn{6}{|l|}{$\begin{array}{l}\text { Mean age of Initiation of Substance } \\
\text { Use (Years), (SD) }\end{array}$} \\
\hline Tobacco $^{+}$ & $13.1(5.5)$ & $13.4(4.0)$ & $13.2(6.7)$ & $12.8(4.1)$ & 0.92 \\
\hline Alcohol $^{+}$ & $13.8(8.7)$ & $12.5(3.9)$ & $15.1(11.4)$ & $12.7(4.6)$ & 0.55 \\
\hline Cocaine $^{+}$ & $18.7(5.4)$ & $20.7(6.1)$ & $18.0(6.1)$ & $18.2(3.8)$ & 0.42 \\
\hline Marijuana $^{+}$ & $14.5(4.5)$ & $14.5(3.0)$ & $15.1(5.5)$ & $13.4(3.4)$ & 0.47 \\
\hline Heroin $^{+}$ & $24.7(9.8)$ & $26.4(12.6)$ & $25.0(9.2)$ & $23.2(9.1)$ & 0.74 \\
\hline Prescription Opioids $^{+}$ & $18.4(7.4)$ & $17.3(5.5)$ & $19.1(7.7)$ & $17.8(7.9)$ & 0.41 \\
\hline Illicit use of Buprenorphine & $25.6(10.6)$ & $23.6(10.7)$ & $25.5(9.6)$ & $26.9(13.1)$ & 0.87 \\
\hline Methamphetamines/Amphetamines ${ }^{+}$ & $26.6(8.9)$ & $29.5(9.4)$ & $24.9(8.3)$ & $27.8(9.4)$ & 0.31 \\
\hline Benzodiazepines & $21.4(9.3)$ & $19.8(7.9)$ & $22.5(10.4)$ & $20.5(8.6)$ & 0.79 \\
\hline $\begin{array}{l}\text { Mean number substances used } \\
\text { (Past } 30 \text { days), (SD) }\end{array}$ & $2.5(1.5)$ & $2.2(2.0)$ & $2.5(1.2)$ & $2.7(1.7)$ & 0.64 \\
\hline Ever Inject Drugs (\%Yes, Lifetime) & 75.8 & 84.6 & 66.7 & 85.0 & 0.26 \\
\hline Mean Age First IDU (SD) ${ }^{+}$ & $24.9(9.2)$ & $23.5(8.5)$ & $24.5(9.0$ & $26.3(10.1)$ & 0.72 \\
\hline \multicolumn{6}{|c|}{ 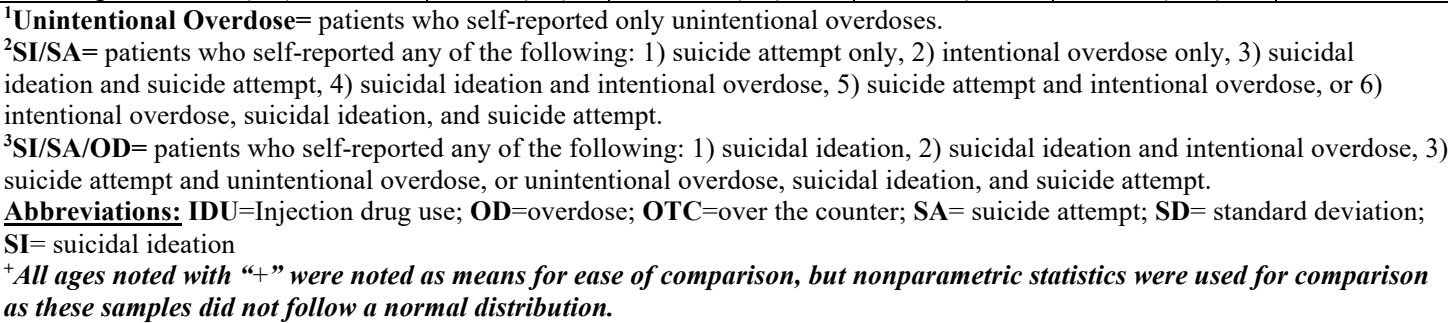 } \\
\hline
\end{tabular}




\subsection{Supplemental Table Chapter 4: Not Included in Analysis}

\begin{tabular}{|c|c|c|c|c|c|}
\hline \multicolumn{6}{|c|}{ Supplemental Table: Life Events Checklist Item Analysis (Chapter 4) } \\
\hline & $\begin{array}{c}\text { Overall } \\
(n=66)\end{array}$ & $\begin{array}{c}\text { Unintentional OD } \\
(n=13)\end{array}$ & $\begin{array}{c}\text { SI/SA } \\
(n=33)\end{array}$ & $\begin{array}{c}\text { SI/SA/OD } \\
(\mathbf{n}=\mathbf{2 0})\end{array}$ & p-value \\
\hline \multicolumn{6}{|l|}{ Past Year Event (\% Yes) } \\
\hline Relationship Dissolution & 59.1 & 86.6 & 60.6 & 40.0 & 0.04 \\
\hline Natural Disaster & 4.6 & 0.0 & 6.1 & 5.0 & 1.0 \\
\hline Fire & 7.6 & 7.7 & 3.0 & 15.0 & 0.27 \\
\hline Work Accident & 4.6 & 0.0 & 6.1 & 5.0 & 1.0 \\
\hline Car Accident & 13.6 & 7.7 & 9.1 & 25.0 & 0.23 \\
\hline Exposure to Toxic Substance & 18.2 & 15.4 & 18.2 & 20.0 & 1.0 \\
\hline Physical Assault & 37.9 & 30.8 & 36.4 & 45.0 & 0.75 \\
\hline Assault with a Weapon & 13.6 & 7.7 & 15.2 & 15.0 & 0.90 \\
\hline Sexual Assault & 10.6 & 0.0 & 15.2 & 10.0 & 0.38 \\
\hline Unwanted Sexual Experiences & 15.2 & 7.7 & 18.2 & 15.0 & 0.90 \\
\hline Combat Exposure & - & - & - & - & - \\
\hline Captivity & 15.2 & 7.7 & 12.1 & 25.0 & 0.42 \\
\hline Severe Suffering & 34.9 & 30.8 & 27.3 & 50.0 & 0.26 \\
\hline Violent Death (witnessed) & 4.6 & 0.0 & 6.1 & 5.0 & 1.0 \\
\hline Unexpected Death (witnessed) & 15.2 & 15.4 & 21.1 & 5.0 & 0.35 \\
\hline Caused Harm or Death & 7.6 & 0.0 & 9.1 & 10.0 & 0.70 \\
\hline Illness & 34.9 & 38.5 & 27.3 & 45.0 & 0.38 \\
\hline Misc. Stressful Experiences & 50.0 & 69.2 & 48.5 & 40.0 & 0.28 \\
\hline \multicolumn{6}{|c|}{$\begin{array}{l}\text { Supplemental Table: Response variables were recoded to yes or no. "Yes," was defined as having experienced or witnessed any of the } \\
\text { events listed on the life events check list. All other responses were coded as "No." This was done due to small sample size and overall } \\
\text { distribution of response rates. }\end{array}$} \\
\hline
\end{tabular}

